\title{
On Stokes operators with variable viscosity in bounded and unbounded domains
}

\author{
Helmut Abels • Yutaka Terasawa
}

Received: 3 April 2008 / Revised: 14 August 2008 / Published online: 4 December 2008

(C) The Author(s) 2008. This article is published with open access at Springerlink.com

\begin{abstract}
We consider a generalization of the Stokes resolvent equation, where the constant viscosity is replaced by a general given positive function. Such a system arises in many situations as linearized system, when the viscosity of an incompressible, viscous fluid depends on some other quantities. We prove that an associated Stokes-like operator generates an analytic semi-group and admits a bounded $H_{\infty}$-calculus, which implies the maximal $L^{q}$-regularity of the corresponding parabolic evolution equation. The analysis is done for a large class of unbounded domains with $W_{r}^{2-\frac{1}{r}}$-boundary for some $r>d$ with $r \geq q, q^{\prime}$. In particular, the existence of an $L^{q}$-Helmholtz projection is assumed.
\end{abstract}

Mathematics Subject Classification (2000) 35Q30 - 76D07 · 47A60 - 47F05

\section{Introduction and assumptions}

We consider the following Stokes-like resolvent system

$$
\begin{aligned}
& \lambda v-\operatorname{div}(2 v(x) D v)+\nabla p=f \quad \text { in } \Omega, \\
& \operatorname{div} v=g \text { in } \Omega \text {, } \\
& \left.v\right|_{\Gamma_{1}}=0 \text { on } \Gamma_{1}, \\
& \left.n \cdot T(v, p)\right|_{\Gamma_{2}}=a \text { on } \Gamma_{2} \text {, }
\end{aligned}
$$

\footnotetext{
H. Abels $(\varangle)$

Max Planck Institute for Mathematics in the Sciences, Inselstr. 22, 04103 Leipzig, Germany

e-mail: abels@mis.mpg.de

Y. Terasawa

Department of Mathematics, Hokkaido University, Kita 10, Nishi 8, Kita-Ku,

Sapporo, Hokkaido 060-0810, Japan

e-mail: yutaka@math.sci.hokudai.ac.jp
} 
where $v: \Omega \rightarrow \mathbb{R}^{d}$ is the velocity of the fluid, $p: \Omega \rightarrow \mathbb{R}$ is the pressure,

$$
T(v, p)=2 v(x) D v-p I
$$

is the stress tensor, $D v=\frac{1}{2}\left(\nabla v+\nabla v^{T}\right), v: \Omega \rightarrow(0, \infty)$ is a variable viscosity coefficient, and $\Omega \subseteq \mathbb{R}^{d}, d \geq 2$, is a suitable domain with boundary $\partial \Omega=\Gamma_{1} \cup \Gamma_{2}$ consisting of two closed, disjoint (possibly empty) components $\Gamma_{j}, j=1,2$. Moreover, we denote $S(v)=2 v D v$.

In the case that $v(x)=v_{0} \in(0, \infty)$ is independent of $x$ the latter system was extensively studied in many kinds of different domains relevant for mathematical fluid mechanics. The system arises as linear system of the non-stationary Navier-Stokes equations for incompressible fluids after Laplace transformation, which replaces the derivative in time by a spectral parameter $\lambda$. But in many situations the viscosity $v$ of an incompressible fluid depends on some quantities as, e.g., the shear rate $|D v|$ in the case of some non-Newtonian fluids, cf. e.g., Malek et al. [40], or a concentration $c$ as in the case of diffuse interface models for free boundary value problems, cf. e.g., Abels [4].

First results on general non-stationary Stokes systems, including the latter case of variable viscosity, were obtained by Solonnikov $[49,50]$ in $L^{q}-$ Sobolev spaces and weighted Hölder spaces and Bothe and Prüß [20] in $L^{q}$-Sobolev spaces, where applications to non-Newtonian fluids are treated as well. Some results on the Stokes system with variable viscosity in $L^{2}$-Sobolev spaces can also be found in $[4,13]$, where applications to a diffuse interface model are also treated. Finally, we note that Ladyženskaja and Solonnikov [42] and later Danchin [22] obtained results for a similar non-stationary Stokes system with variable density instead of variable viscosity.

The purpose of the present contribution is to study the (generalized) Stokes resolvent equation (1.1)-(1.4) and an associated Stokes operator in $L^{q}$-Sobolev spaces, $1<q<\infty$, in a class of general bounded and unbounded domain, which is similar to the class in [8] and which covers most cases studied so far in the case of constant viscosity. More precisely, we will show that the associated Stokes operator $-A_{q}$, defined below, generates an analytic semi-group $e^{-t A_{q}}, t \geq 0$, on $L^{q}(\Omega)^{d}$. We will even show that $A_{q}$ admits a bounded $H_{\infty}$-calculus in the sense of McIntosh [44]. This has several strong implication as will be explained below.

In the case of constant viscosity the boundedness and analyticity of the Stokes semi-group was proved by Giga [32] for the case of bounded domains, Borchers and Sohr [18] and Borchers and Varnhorn [19] for the case of an exterior domain, and Farwig and Sohr [31] in the case of an aperture domain. We refer to Farwig and Sohr [30] for a general approach to unbounded and bounded domains. The case of infinite layers and layer-like domains were discussed by Abe and Shibata [2,3], Abe [1], Abels and Wiegner [14], and Abels [10,12]. The case of an infinite cylinder was treated by Farwig and Ri $[28,29]$. For the proof of bounded imaginary powers or a bounded $H^{\infty}$-calculus in the latter domains we refer to Giga [33], Giga and Sohr [34], Noll and Saal [46], Farwig and Ri [28], and Abels [5,7,8,11]. Finally, we refer to Farwig, Kozono, and Sohr [27] for results on the Stokes system in general unbounded domains with uniform $C^{2}$-boundary in Sobolev spaces based on $L^{q}(\Omega) \cap L^{2}(\Omega)$ if $2 \leq q<\infty$ and $L^{q}(\Omega)+L^{2}(\Omega)$ if $1<q \leq 2$. 
Before we present our main results we state the assumptions on the domain and related function spaces:

Assumption 1 Let $1<q<\infty$, let $d<r_{1}, r_{2} \leq \infty$ such that $q, q^{\prime} \leq \min \left(r_{1}, r_{2}\right)$, and let $v(x)=v_{\infty}+v^{\prime}(x)$ such that $v^{\prime}(x) \in W_{r_{1}}^{1}(\Omega)$ and $v(x) \geq v_{0}>0$ for all $x \in \Omega$. Moreover, let $\Omega \subseteq \mathbb{R}^{d}, d \geq 2$, be a domain and $\partial \Omega=\Gamma_{1} \cup \Gamma_{2}$ with $\Gamma_{1}, \Gamma_{2}$ closed and disjoint satisfying the following conditions:

(A1) There is a finite covering of $\bar{\Omega}$ with relatively open sets $U_{j}, j=1, \ldots, m$, such that $U_{j}$ coincides (after rotation) with a relatively open set of $\overline{\mathbb{R}_{\gamma_{j}}^{d}}$, where $\mathbb{R}_{\gamma_{j}}^{d}:=\left\{\left(x^{\prime}, x_{d}\right) \in \mathbb{R}^{d}: x_{d}>\gamma_{j}\left(x^{\prime}\right)\right\}, \gamma_{j} \in W_{r_{2}}^{2-\frac{1}{r} 2}\left(\mathbb{R}^{d-1}\right)$. Moreover, suppose that there are cut-off functions $\varphi_{j}, \psi_{j} \in C_{b}^{\infty}(\bar{\Omega}), j=1, \ldots, m$, such that $\varphi_{j}$, $j=1, \ldots, m$, is a partition of unity, $\psi_{j} \equiv 1$ on $\operatorname{supp} \varphi_{j}$, and $\operatorname{supp} \psi_{j} \subset U_{j}$, $j=1, \ldots, m$.

(A2) For every $f \in L^{s}(\Omega)^{d}, s=q, q^{\prime}$, there is a unique decomposition $f=f_{0}+\nabla p$ with $f_{0} \in J_{S}(\Omega)$ and $p \in \dot{W}_{s, \Gamma_{2}}^{1}(\Omega)$ where

$$
\begin{aligned}
J_{s}(\Omega) & :={\overline{\left\{f \in C_{(0)}^{\infty}\left(\Omega \cup \Gamma_{2}\right)^{d}: \operatorname{div} f=0\right\}}}^{L^{s}(\Omega)}, \\
\dot{W}_{s, \Gamma_{2}}^{1}(\Omega) & :=\left\{p \in \dot{W}_{s}^{1}(\Omega):\left.p\right|_{\Gamma_{2}}=0\right\} .
\end{aligned}
$$

(A3) For every $p \in \dot{W}_{s, \Gamma_{2}}^{1}(\Omega), s=q, q^{\prime}$, there is a decomposition $p=p_{1}+p_{2}$ such that $p_{1} \in W_{s}^{1}(\Omega)$ with $\left.p_{1}\right|_{\Gamma_{2}}=0, p_{2} \in L_{\text {loc }}^{s}(\bar{\Omega})$ with $\nabla p_{2} \in W_{s}^{1}(\Omega)$ and $\left\|\left(p_{1}, \nabla p_{2}\right)\right\|_{W_{s}^{1}(\Omega)} \leq C\|\nabla p\|_{s}$.

Remark 1 1. It is easy to see that (A1) is fulfilled for all kinds of domains with $W_{r_{2}}^{2-\frac{1}{r_{2}}}$-boundary mentioned above. The assumption (A2) guarantees the existence of a Helmholtz-projection adapted to the boundary conditions (1.3)-(1.4). We refer to $[10,26,30,31,45,48]$ for the validity of the Helmholtz decomposition for these types of domains for the case $\Gamma_{2}=\emptyset$. Moreover, (A3) is a technical condition needed in the Sect. 6 below. It is used to overcome the difficulty that multiplication with not compactly supported cut-off functions is not continuous on $\dot{W}_{q, \Gamma}^{1}(\Omega)$ in general. The condition is satisfied if the following extension property is valid: For every $p \in \dot{W}_{q}^{1}(\Omega)$ there is an extension $\tilde{p} \in \dot{W}_{q}^{1}\left(\mathbb{R}^{d}\right)$ such that $\left.\tilde{p}\right|_{\Omega}=p$ and $\|\nabla \tilde{p}\|_{q} \leq C\|\nabla p\|_{q}$. This is the case for every $(\varepsilon, \infty)$-domain, cf. [21], in particular, for exterior domains. This extension property does not hold for layer-like domains, cf. [10, Sect. 2.4]. Nevertheless (A3) is also valid in layer-like domains, cf. [10, Lemma 2.4].

2. Let us comment on the regularity assumptions on $v$ and $\partial \Omega$. First of all, $v \in$ $W_{r_{1}}^{1}(\Omega)$ with $r_{1}>d$ implies that multiplication with $v$ defines a continuous mapping on $W_{q}^{1}(\Omega)$ for every $1 \leq q<r_{1}$, cf. Lemma 1 below. In particular, this implies that $\operatorname{div}(2 v D v) \in L^{q}(\Omega)^{d}$ for every $v \in W_{q}^{2}(\Omega)^{d}$ and $1 \leq q<r_{1}$. Since we will partly argue by duality, we also require $q^{\prime}<r_{1}$. Moreover, since $r_{1}>d$, $W_{r_{1}}^{1}(\Omega) \hookrightarrow C^{1-\frac{d}{r_{1}}}(\bar{\Omega})$. Therefore $\operatorname{div}(2 v D v)=v(\Delta v+\nabla \operatorname{div} v)+\nabla v \cdot D v$, 
where $\nabla v \cdot D v$ is of lower order and the principal part $v(\Delta v+\nabla \operatorname{div} v)$ has Hölder continuous coefficients. The latter property is essential to apply pseudodifferential operator methods with symbols that are Hölder continuous with respect to the space variable $x$. Concerning the boundary regularity, we note that every $\gamma \in W_{r_{2}}^{2-\frac{1}{r_{2}}}\left(\mathbb{R}^{d-1}\right)$ can be extended to some $\Gamma \in W_{r_{2}}^{2}\left(\mathbb{R}_{+}^{d}\right)$, which is then used to build suitable coordinate transformations. After transforming the (reduced) Stokes system on $\mathbb{R}_{\gamma}^{d}$ to $\mathbb{R}_{+}^{d}$, the principal part of transformed differential operators will have coefficients depending on $\nabla \Gamma \in W_{r_{2}}^{1}\left(\mathbb{R}_{+}^{d}\right)$, which embeds again to a space of Hölder continuous functions since $r_{2}>d$. Hence multiplication by $\nabla \Gamma$ plays a similar role as multiplication by $v$ and that is where the conditions related to $r_{1}, r_{2}$ in the assumptions come from. Finally, let us note that, if $\partial \Omega$ is compact, $C^{1,1}(\partial \Omega) \hookrightarrow W_{r^{\prime}}^{2-\frac{1}{r^{\prime}}}(\partial \Omega) \hookrightarrow W_{r}^{2-\frac{1}{r}}(\partial \Omega)$ for all $1 \leq r \leq r^{\prime} \leq \infty$. Therefore the local regularity decreases if $r_{1}, r_{2}$ are chosen smaller and the case $r_{1}=r_{2}=\infty$ corresponds to the strongest regularity assumptions. On the other hand, the smaller $r_{1}, r_{2}$ are chosen, the more restrictive the condition $q, q^{\prime}<\min \left(r_{1}, r_{2}\right)$ gets.

In some parts of the paper we will assume additionally that the following assumption holds:

(A4) There is some $R>0$ such that for every $\lambda \in \Sigma_{\delta}$ with $|\lambda| \geq R$ there is no non-trivial solution $g \in W_{q}^{1}(\Omega)$ with $\left.g\right|_{\Gamma_{2}}=0$ of

$$
\lambda(g, \varphi)_{\Omega}+(\nu \nabla g, \nabla \varphi)_{\Omega}=0 \text { for all } \varphi \in W_{q^{\prime}, \Gamma_{2}}^{1}(\Omega) .
$$

Here $W_{q^{\prime}, \Gamma_{2}}^{1}(\Omega)=\left\{\varphi \in W_{q^{\prime}}^{1}(\Omega):\left.\varphi\right|_{\Gamma_{2}}=0\right\}$. We will show later that (A4) is a consequence of Assumption 1, cf. Lemma 14 below.

The reduced Stokes operator $A_{q}$ on $L^{q}(\Omega)^{d}$ is defined as

$$
\begin{aligned}
A_{q} v & =-\operatorname{div}(v \nabla v)+\nabla P v-\nabla v^{T} \nabla v^{T} \\
\mathcal{D}\left(A_{q}\right) & =\left\{v \in W_{q}^{2}(\Omega)^{d}:\left.v\right|_{\Gamma_{1}}=0,\left.T_{1}^{\prime} v\right|_{\Gamma_{2}}=0\right\},
\end{aligned}
$$

where $T_{1}^{\prime} v$ is defined by

$$
\left(T_{1}^{\prime} v\right)_{\tau}=\left.(n \cdot S(v))_{\tau}\right|_{\Gamma_{2}}, \quad\left(T_{1}^{\prime} v\right)_{n}=\left.v \operatorname{div} v\right|_{\Gamma_{2}} .
$$

Here $f_{\tau}, f_{n}$ denotes the tangential, normal component, resp., of a vector field $f$ at the boundary $\partial \Omega$. Moreover, $P v \equiv p_{1} \in \dot{W}_{q}^{1}(\Omega)$ with $\left.p_{1}\right|_{\Gamma_{2}} \in W_{q}^{1-\frac{1}{q}}\left(\Gamma_{2}\right)$ is defined as the solution of

$$
\begin{gathered}
\left(\nabla p_{1}, \nabla \varphi\right)_{\Omega}=(v(\Delta-\nabla \operatorname{div}) v, \nabla \varphi)_{\Omega}+(D v, 2 \nabla v \otimes \nabla \varphi)_{\Omega}, \\
\left.p_{1}\right|_{\Gamma_{2}}=2 \nu \partial_{n} v_{n}
\end{gathered}
$$

for all $\varphi \in \dot{W}_{q^{\prime}, \Gamma_{2}}^{1}(\Omega)=\left\{\varphi \in \dot{W}_{q^{\prime}}^{1}(\Omega):\left.\varphi\right|_{\Gamma_{2}}=0\right\}$. Note that the righthand-side of (1.8) defines a bounded linear functional on $\dot{W}_{q^{\prime}, \Gamma_{2}}^{1}(\Omega)$. The existence of a solution 
of (1.8)-(1.9) that is unique (up to a constant if $\Gamma_{2}=\emptyset$ ) follows from the existence of a unique Helmholtz decomposition, i.e., (A2), cf. Lemma 2 below. Then $P: W_{q}^{2}(\Omega)^{d} \rightarrow\left\{p \in \dot{W}_{q}^{1}(\Omega):\left.p\right|_{\Gamma_{2}} \in W_{q}^{1-\frac{1}{q}}\left(\Gamma_{2}\right)\right\}$ is a bounded linear operator.

The connection to the original system is discussed in Sect. 3 below. We note that the definition of $A_{q}$, in particular the lower order term $\nabla v^{T} \nabla v^{T}$, is chosen such that for all $u \in \mathcal{D}\left(A_{q}\right)$ with $\operatorname{div} u=0$ and $v \in W_{q^{\prime}}^{1}(\Omega)$ with $\left.v\right|_{\Gamma_{1}=0}$, $\operatorname{div} v=0$

$$
\begin{aligned}
\left(A_{q} u, v\right)_{\Omega} & =(-\operatorname{div}(2 v D u), v)_{\Omega}+(\nabla P u, v)_{\Omega} \\
& =(2 v D u, D v)_{\Omega}-\left(n \cdot S(u) \cdot n, v_{n}\right)_{\Gamma_{2}}+\left(2 v \partial_{n} u_{n}, v_{n}\right)_{\Gamma_{2}} \\
& =(2 v D u, D v)_{\Omega}
\end{aligned}
$$

holds.

The main result is the following:

Theorem 1 Let $\Omega \subseteq \mathbb{R}^{d}, d \geq 2, \delta \in(0, \pi)$, and $q, r_{1}, r_{2}$ be as in Assumption 1 . Then there is some $R>0$ such that $\left(\lambda+A_{q}\right)^{-1}$ exists and

$$
\left\|\left(\lambda+A_{q}\right)^{-1}\right\|_{\mathcal{L}\left(L^{q}(\Omega)\right)} \leq \frac{C_{q, \delta}}{1+|\lambda|}
$$

for all $\lambda \in \Sigma_{\delta}$ with $|\lambda| \geq R$. Moreover,

$$
\left\|\int_{\Gamma_{R}} h(-\lambda)\left(\lambda+A_{q}\right)^{-1} d \lambda\right\|_{\mathcal{L}\left(L^{q}(\Omega)\right)} \leq C_{q, \delta}\|h\|_{L^{\infty}\left(\Sigma_{\pi-\delta}\right)}
$$

for every $h \in H_{\infty}(\delta)$, where $\Gamma_{R}=\Gamma \backslash \overline{B_{R}(0)}$ and $H_{\infty}(\delta)$ denotes the Banach algebra of all bounded holomorphic functions $h: \Sigma_{\pi-\delta} \rightarrow \mathbb{C}$. In particular, for every $c \in \mathbb{R}$ and $0<\delta^{\prime} \leq \delta$ such that $c+\Sigma_{\delta^{\prime}} \subset \rho\left(-A_{q}\right)$ the shifted reduced Stokes operator $c+A_{q}$ admits a bounded $H_{\infty}$-calculus with respect to $\delta^{\prime}$, i.e.,

$$
h\left(c+A_{q}\right):=\frac{1}{2 \pi i} \int_{\Gamma} h(-\lambda)\left(\lambda+c+A_{q}\right)^{-1} d \lambda
$$

is a bounded operator satisfying

$$
\left\|h\left(c+A_{q}\right)\right\|_{\mathcal{L}\left(L^{q}(\Omega)\right)} \leq C_{q, \delta}\|h\|_{L^{\infty}\left(\Sigma_{\pi-\delta}\right)}
$$

for all $h \in H_{\infty}\left(\delta^{\prime}\right)$.

We note that in order to prove (1.14) for all $h \in H_{\infty}(\delta)$ it is sufficient to show the estimate for $h \in H(\delta)$, which consists of all $h \in H_{\infty}(\delta)$ such that 


$$
|h(z)| \leq C \frac{|z|^{S}}{1+|z|^{2 s}} \quad \text { for all } z \in \Sigma_{\pi-\delta}
$$

for some $s>0$, cf. Denk, Hieber, and Prüss [24, Sect. 2.4].

We note that (A2) is always true in the case of a bounded domain because of [48] and since $W_{r_{2}}^{2-\frac{1}{r_{2}}}\left(\mathbb{R}^{d-1}\right) \hookrightarrow C^{1}\left(\mathbb{R}^{d-1}\right)$ if $r_{2}>d$. Moreover, (A1) is trivially true and (A3) is valid too by Poincaré's inequality. In this case we obtain:

Theorem 2 Let $\Omega \subseteq \mathbb{R}^{d}, d \geq 2, \delta \in(0, \pi)$, and $q, r_{1}, r_{2}$ be as in Assumption 1 . Moreover, assume that $\Omega$ is bounded and that $\Gamma_{1} \neq \emptyset$. Then $\Sigma_{\delta} \cup\{0\} \subseteq \rho\left(-A_{q}\right)$ and

$$
\left\|\left(\lambda+A_{q}\right)^{-1}\right\|_{\mathcal{L}\left(L^{q}(\Omega)\right)} \leq \frac{C_{q, \delta}}{1+|\lambda|}
$$

for all $\lambda \in \Sigma_{\delta} \cup\{0\}$. Moreover, $A_{q}$ admits a bounded $H_{\infty}$-calculus with respect to $\delta$.

Finally, note that, if $c+A_{q}$ admits a bounded $H_{\infty}$-calculus with respect to $0<\delta<$ $\pi$, then, choosing $h(\lambda)=\lambda^{i y}, y \in \mathbb{R}$, above, one obtains that $c+A_{q}$ has bounded imaginary powers $\left(c+A_{q}\right)^{i y}$, which satisfy

$$
\left\|\left(c+A_{q}\right)^{i y}\right\|_{\mathcal{L}\left(L^{q}(\Omega)^{d}\right)} \leq C e^{|y|(\pi-\delta)},
$$

where we note that $\sup _{\lambda \in \Sigma_{\delta-\pi}}\left|\lambda^{i y}\right|=e^{|y|(\pi-\delta)}$. This has two important consequences, which we summarize in the following. The first one concerns so-called maximal regularity of the reduced Stokes operator $A_{q}$ and follows from the well-known result due to Dore and Venni [25, Theorem 3.2] and its extension by Giga and Sohr [35, Theorem 2.1].

Theorem 3 Let $1<p<\infty, 0<T \leq \infty$, and let $\Omega, q$ be as in Assumption 1 . Moreover, let $c \in \mathbb{R}$ be such that $c+A_{q}$ is invertible and admits a bounded $H_{\infty^{-}}$ calculus. Then for every $f \in L^{p}\left(0, T ; L^{q}(\Omega)^{d}\right)$ there is a unique solution $u \in$ $W_{p}^{1}\left(0, T ; L_{\sigma}^{q}(\Omega)\right) \cap L^{p}\left(0, T ; \mathcal{D}\left(A_{q}\right)\right)$ of

$$
\begin{aligned}
& u^{\prime}(t)+\left(c+A_{q}\right) u(t)=f(t), \quad 0<t<T, \\
& u(0)=0
\end{aligned}
$$

Moreover,

$$
\left\|u^{\prime}\right\|_{L^{p}\left(0, T ; L^{q}\right)}+\left\|\left(c+A_{q}\right) u\right\|_{L^{p}\left(0, T ; L^{q}\right)} \leq C\|f\|_{L^{p}\left(0, T ; L^{q}\right)},
$$

where $C$ does not depend on $T$.

In particular, in the case of a bounded domain with $W_{r_{2}}^{2-\frac{1}{r_{2}}}$-boundary the latter theorem implies that $A_{q}$ has maximal regularity on $L^{q}(\Omega)^{d}$ for all $1<q<\infty$ with $q, q^{\prime} \leq \min \left(r_{1}, r_{2}\right)$, where $d<r_{1}, r_{2} \leq \infty$ and $v \in W_{r_{1}}^{1}(\Omega)$.

As a second application we note that the boundedness of $\left(c+A_{q}\right)^{i y}$ and (1.15) can be used to characterize the domain of the fractional powers $\left(c+A_{q}\right)^{\alpha}, 0<\alpha<1$, as 


$$
\mathcal{D}\left(\left(c+A_{q}\right)^{\alpha}\right)=\left(L^{q}(\Omega)^{d}, \mathcal{D}\left(A_{q}\right)\right)_{[\alpha]},
$$

where $(., .)_{[\alpha]}$ denotes the complex interpolation functor, cf. [34, Proposition 6.1]. Here again $c \in \mathbb{R}$ is such that $c+A_{q}$ is invertible and admits a bounded $H^{\infty}$-calculus.

The proof of Theorem 1 is based on a similar result for a bent half-space $\mathbb{R}_{\gamma}^{d}$, cf. Theorem 4 below, which is obtained by constructing a suitable approximation of the resolvent $\left(\lambda+A_{q}\right)^{-1}$. The latter construction uses the technique developed in [11], combined with newer results on the general calculus of pseudodifferential boundary value problems studied in [9], adapted to the case of variable viscosity.

The structure of the article is as follows: In Sect. 2 we summarize some preliminaries and some notation. In Sect. 3 we discuss how the pressure $p$ and the divergence equation can be eliminated from (1.1)-(1.4). This uses the ideas of Grubb and Solonnikov, cf. e.g., [39]. The reduced system contains the non-local operator $P v$, which can be approximated naturally in the class of pseudodifferential boundary value problems going back to Boutet de Monvel [23] and developed further by Grubb [37] to parameter-dependent operators and by the first author to the case of non-smooth symbols $[6,9,11]$. Section 4 is devoted to some needed results on coordinate transformation and the change of operators under coordinate transformation. The main step is done in Sect. 5, where a suitable result for a bent half-space is proved using the previously mentioned techniques. Using the latter result, Theorem 1 is proved in Sect. 6. Finally, the result for bounded domains, i.e., Theorem 2, is proved in Sect. 7.

\section{Preliminaries}

First of all, $\mathbb{N}$ will denote the set of natural numbers (without 0 ) and $\mathbb{N}_{0}:=\mathbb{N} \cup\{0\}$. Moreover, we denote $\mathbb{R}_{+}^{d}=\left\{x \in \mathbb{R}^{d}: x_{d}>0\right\}, a \otimes b=\left(a_{i} b_{j}\right)_{i, j=1}^{d}$ for $a, b \in \mathbb{R}^{d}, e_{j}$ denotes the $j$ th canonical unit vector, and $[A, B]=A B-B A$ the commutator of two operators $A, B$. We frequently use the decomposition $x=\left(x^{\prime}, x_{d}\right)$ of $x \in \mathbb{R}^{d}$, where $x^{\prime} \in \mathbb{R}^{d-1}$ denotes the first $(d-1)$-components of $x$. Moreover, we identify $\mathbb{R}^{d-1}$ with $\partial \mathbb{R}_{+}^{d}=\mathbb{R}^{d-1} \times\{0\}$ and $x^{\prime} \in \mathbb{R}^{d-1}$ with $\left(x^{\prime}, 0\right)$ in the following. For completeness, we note that, if $v: \Omega \rightarrow \mathbb{R}^{d}$ is a suitable vector field, then $\nabla v=\left(\partial_{j} v_{k}\right)_{j, k=1}^{d}$. Moreover, if $A: \Omega \rightarrow \mathbb{R}^{d \times d}$ is suitable, then $\operatorname{div} A=\left(\sum_{j=1}^{d} \partial_{j} a_{j k}\right)_{k=1}^{d}$, where $A=\left(a_{j k}\right)_{j, k=1}^{d}$.

If $X$ is a Banach space and $X^{\prime}$ is its dual, then

$$
\langle f, g\rangle \equiv\langle f, g\rangle_{X^{\prime}, X}=f(g), \quad f \in X^{\prime}, g \in X,
$$

denotes the duality product.

Let $M \subseteq \mathbb{R}^{d}, d \geq 2$. Then $C_{b}^{k}(M), k \in \mathbb{N}_{0}$, denotes the set of all $k$-times continuously differentiable functions $f: M \rightarrow \mathbb{C}$ such that $f$ and all its derivatives are bounded. Moreover, $C_{b}^{\infty}(M)=\cap_{k \in \mathbb{N}} C_{b}^{k}(M)$ and $C_{(0)}^{\infty}(M)$ is the set of all $f \in$ $C^{\infty}(M)$ with supp $f \subseteq M$ compact, and, if $\Omega \subset \mathbb{R}^{d}$ is a domain, then $C_{0}^{\infty}(\Omega) \equiv$ $C_{(0)}^{\infty}(\Omega)$. The usual Lebesgue-space with respect to the Lebesgue measure on $\Omega$ and the $(d-1)$-dimensional surface measure on $\partial \Omega$ will be denoted by $L^{q}(\Omega), L^{q}(\partial \Omega)$, resp., $1 \leq q \leq \infty$. Moreover, we use the abbreviations $\|\cdot\|_{q} \equiv\|\cdot\|_{L^{q}(\Omega)}$ and $\|\cdot\|_{q, \partial \Omega} \equiv$ 
$\|\cdot\|_{L^{q}(\partial \Omega)}$. Furthermore, $L_{\text {loc }}^{q}(\bar{\Omega}), 1 \leq q \leq \infty$, is defined as the space of all $f: \Omega \rightarrow$ $\mathbb{C}$ such that $f \in L^{q}(B \cap \Omega)$ for all balls $B$ with $B \cap \Omega \neq \emptyset$. The usual scalar product on $L^{2}(M)$ is denoted by $(., .)_{M}$ for $M=\Omega, \partial \Omega$. Finally, if $\omega: \Omega \rightarrow(0, \infty)$, then $L^{p}(\Omega ; \omega)$ denotes the $L^{p}$-space with respect to the measure $\omega(x) d x$.

In the following the usual Sobolev-Slobodeckij spaces based on $L^{q}(\Omega), 1<q<$ $\infty$, are denoted by $W_{q}^{s}(\Omega)$ and $W_{q}^{s}(M), s \geq 0$, with norms $\|\cdot\|_{s, q}$ and $\|\cdot\|_{s, q, \partial \Omega}$, respectively, cf. e.g., [15], where $M \subset \mathbb{R}^{d}$ is a $(d-1)$-dimensional sufficiently smooth manifold. We note that, if $0<s<1$, then it is sufficient to assume that $M$ is a $C^{1}$-manifold to define $W_{q}^{s}(M)$ in the usual way. Moreover, $W_{q, 0}^{m}(\Omega), m \in \mathbb{N}$, denotes the closure of $C_{0}^{\infty}(\Omega)$ in $W_{q}^{m}(\Omega)$ and

$$
W_{q}^{-m}(\Omega):=\left(W_{q^{\prime}, 0}^{m}(\Omega)\right)^{\prime}, W_{q, 0}^{-m}(\Omega):=\left(W_{q^{\prime}}^{m}(\Omega)\right)^{\prime}, W_{q}^{-s}(\partial \Omega):=\left(W_{q^{\prime}}^{s}(\partial \Omega)\right)^{\prime}
$$

for $m \in \mathbb{N}$ and $s>0$, where $\frac{1}{q}+\frac{1}{q^{\prime}}=1$.

Finally, the homogeneous Sobolev space of order 1 is defined as

$$
\dot{W}_{q}^{1}(\Omega):=\left\{p \in L_{\mathrm{loc}}^{q}(\bar{\Omega}): \nabla p \in L^{q}(\Omega)\right\}
$$

normed by $\|\nabla \cdot\|_{q}$, where functions, which differ by a constant, are identified.

Additionally, $\mathcal{F}$ and $\mathcal{F}^{-1}$ denote the Fourier and inverse Fourier transformation,

$$
\begin{aligned}
& \mathcal{F}[f](\xi):=\hat{f}(\xi):=\int_{\mathbb{R}^{d}} e^{-i x \cdot \xi} f(x) d x, \\
& \mathcal{F}^{-1}[f](x):=\check{f}(x):=\int_{\mathbb{R}^{d}} e^{i x \cdot \xi} f(\xi) d \xi,
\end{aligned}
$$

defined for a suitable function $f: \mathbb{R}^{d} \rightarrow \mathbb{C}$, where $d \xi:=(2 \pi)^{-d} d \xi$. Note that in the following all integrals with respect to a phase variable $\xi$ will be scaled by $(2 \pi)^{-d}$ as above. Moreover, we will use partial Fourier transformation

$$
\mathcal{F}_{x^{\prime} \mapsto \xi^{\prime}}[f]\left(\xi^{\prime}, x_{d}\right):=f^{\prime}\left(\xi^{\prime}, x_{d}\right):=\int_{\mathbb{R}^{d-1}} e^{-i x^{\prime} \cdot \xi^{\prime}} f\left(x^{\prime}, x_{d}\right) d x^{\prime}
$$

and the conjugate Fourier transformation $\overline{\mathcal{F}}[f](\xi)=\mathcal{F}[f](-\xi)$.

Let $\langle\xi\rangle=\left(1+|\xi|^{2}\right)^{\frac{1}{2}}, \xi \in \mathbb{R}^{d}$, and let $\left\langle D_{x}\right\rangle^{s} \equiv \operatorname{OP}\left(\langle\xi\rangle^{s}\right)=\mathcal{F}^{-1}\left[\langle\xi\rangle^{s} \mathcal{F}[].\right]$, $s \in \mathbb{R}$. Moreover, $\mathcal{S}\left(\mathbb{R}^{d}\right)$ denotes the space of rapidly decreasing smooth functions $f: \mathbb{R}^{d} \rightarrow \mathbb{C}$ and $\mathcal{S}^{\prime}\left(\mathbb{R}^{d}\right)$ denotes the space of tempered distributions. Recall that the Bessel potential space $H_{q}^{s}\left(\mathbb{R}^{d}\right), 1<q<\infty, s \in \mathbb{R}$, is defined as the space of all $f \in \mathcal{S}^{\prime}\left(\mathbb{R}^{d}\right)$ for which $\left\langle D_{x}\right\rangle^{s} f \in L^{q}\left(\mathbb{R}^{d}\right)$, with norm $\|f\|_{H_{q}^{s}}=\left\|\left\langle D_{x}\right\rangle^{s} f\right\|_{L^{q}}$. Moreover, $\mathcal{S}\left(\mathbb{R}^{d} ; X\right)$ and $H_{q}^{s}\left(\mathbb{R}^{d} ; X\right)$ denote the vector-valued variants, where $X$ is a Banach space. As in $[36,38]$, the space $H_{q}^{s}\left(\mathbb{R}_{+}^{d}\right)=r^{+} H_{q}^{s}\left(\mathbb{R}^{d}\right)$ is defined as the space of all distributions of $H_{q}^{s}\left(\mathbb{R}^{d}\right)$ restricted to $\mathbb{R}_{+}^{d}$ equipped with the quotient norm. 
Here and in the following $r^{+} f$ denotes the restriction of $f \in \mathcal{S}^{\prime}\left(\mathbb{R}^{d}\right)$ to $\mathbb{R}_{+}^{d}$. We refer to [17, Chapter 6] for the definition of the usual $B_{q r}^{s}\left(\mathbb{R}^{d}\right), s \in \mathbb{R}, 1 \leq q, r \leq \infty$ and their interpolation properties. Moreover, we note that $B_{q q}^{s}\left(\mathbb{R}^{d}\right)=W_{q}^{s}\left(\mathbb{R}^{d}\right)$ for all $s>0, s \notin \mathbb{N}$, and $1 \leq q<\infty$.

Finally, $\mathcal{C}^{s}\left(\mathbb{R}^{d}\right) \equiv B_{\infty \infty}^{s}\left(\mathbb{R}^{d}\right), s>0$, denotes the Zygmund space and $\mathcal{C}^{s}\left(\mathbb{R}^{d} ; X\right)$ $\equiv B_{\infty \infty}^{s}\left(\mathbb{R}^{d} ; X\right)$ its vector-valued variant for a Banach space $X$. Note that $\mathcal{C}^{s}\left(\mathbb{R}^{d} ; X\right)=$ $C^{s}\left(\mathbb{R}^{d} ; X\right)$ if $s>0$ and $s \notin \mathbb{N}_{0}$, cf. e.g., [16, Equation (5.8)]. Here $C^{s}\left(\mathbb{R}^{d} ; X\right)$ is the space of all $[s]$-times continuously differentiable $f: \mathbb{R}^{d} \rightarrow X$ such that $f$ and all its derivatives are bounded and $\partial_{x}^{\alpha} f,|\alpha|=[s]$, is (uniformly) Hölder continuous of degree $s-[s]$. Here $[s]$ denotes the largest integer not larger than $s$. The space is normed by

$$
\|f\|_{C^{s}\left(\mathbb{R}^{d} ; X\right)}:=\sum_{|\alpha| \leq[s]}\left\|\partial_{x}^{\alpha} f\right\|_{L^{\infty}\left(\mathbb{R}^{d} ; X\right)}+\sum_{|\alpha|=[s]} \sup _{x \neq y} \frac{\left\|\partial_{x}^{\alpha} f(x)-\partial_{x}^{\alpha} f(y)\right\|_{X}}{|x-y|^{s-[s]}}
$$

In the following, let $\Omega$ be a domain as in the Assumption 1. First of all, using the partition of unity assumed in (A2), it is easy to reduce many of the fundamental statements on the Sobolev spaces $W_{q}^{m}(\Omega), m \leq 2$, to a bent half space $\mathbb{R}_{\gamma}^{d}, \gamma \in W_{r_{2}}^{2-\frac{1}{r_{2}}}\left(\mathbb{R}^{d-1}\right)$. Using a suitable coordinate transformation, cf. e.g., Proposition 1 below, the statements for the bent half-space can be proved using the corresponding statement for $\mathbb{R}_{+}^{d}$. In particular, we note that the usual Sobolev embedding theorem for $W_{q}^{1}(\Omega)$ can be proved that way. As a consequence, it is easy to prove the following lemma:

Lemma 1 Let $1<q<\infty$ and $d<r \leq \infty$ such that $q \leq r$ and let $\Omega$ be a domain as in the Assumption 1 with $r_{2}=r$. Then $\pi(f, g)(x):=f(x) g(x)$ defines a continuous, bilinear mapping $\pi: W_{q}^{1}(\Omega) \times W_{r}^{1}(\Omega) \rightarrow W_{q}^{1}(\Omega)$.

Similarly, the interpolation inequality

$$
\|f\|_{W_{q}^{1}(\Omega)} \leq c_{q}\|f\|_{L^{q}(\Omega)}^{\frac{1}{2}}\|f\|_{W_{q}^{2}(\Omega)}^{\frac{1}{2}}
$$

for all $1<q<\infty$ and $f \in W_{q}^{2}(\Omega)$ can be proved. Furthermore, there is a bounded extension operator

$$
E: W_{q}^{1-\frac{1}{q}}(\partial \Omega) \rightarrow W_{q}^{1}(\Omega) \text { such that }\left.E a\right|_{\partial \Omega}=a \text { for all } a \in W_{q}^{1-\frac{1}{q}}(\partial \Omega) .
$$

This extension operator can be easily constructed using the corresponding extension operator for $\mathbb{R}_{+}^{d}$, the partition of unity due to (A1) and suitable coordinate transformations. Note that the corresponding statement for $\dot{W}_{q}^{1}(\Omega)$ and $\dot{W}_{q}^{1-\frac{1}{q}}(\partial \Omega)$ are not true for general unbounded domains; e.g., the statement is not true for an infinite layer, cf. [10, Remark 2.6.1].

Finally, we note that, if $\Omega$ and $q$ are as in Assumption 1, then (A2) implies that for every $f \in L^{q}(\Omega)^{d}$, there is a unique $p \in \dot{W}_{q, \Gamma_{2}}^{1}(\Omega)$ (up to a constant if $\Gamma_{2}=\emptyset$ ) 
depending continuously on $f$ such that

$$
(\nabla p, \nabla \varphi)_{\Omega}=(f, \nabla \varphi)_{\Omega} \text { for all } \varphi \in \dot{W}_{q^{\prime}, \Gamma_{2}}^{1}(\Omega)
$$

Here $p$ is the unique $p \in \dot{W}_{q, \Gamma_{2}}^{1}(\Omega)$ such that $f=f_{0}+\nabla p$ with $f_{0} \in J_{q}(\Omega)$, where we note that

$$
\left(f_{0}, \nabla \varphi\right)_{\Omega}=0 \text { for all } \varphi \in \dot{W}_{q, \Gamma_{2}}^{1}(\Omega)
$$

since it holds for all $f_{0} \in C_{(0)}^{\infty}\left(\Omega \cup \Gamma_{2}\right)$ and the latter space is dense in $J_{q}(\Omega)$ by definition. For the following we define

$$
\dot{W}_{q, \Gamma_{2}}^{-1}(\Omega):=\left(\dot{W}_{q^{\prime}, \Gamma_{2}}^{1}(\Omega)\right)^{\prime} .
$$

Then for every $F \in \dot{W}_{q, \Gamma_{2}}^{-1}$ there is some $f \in L^{q}(\Omega)^{d}$ such that $\|f\|_{L^{q}(\Omega)^{d}} \leq$ $C\|F\|_{\dot{W}_{q, \Gamma_{2}}^{-1}(\Omega)}$ and

$$
\langle F, \varphi\rangle_{\dot{W}_{q, \Gamma_{2}}^{-1}, \dot{W}_{q^{\prime}, \Gamma_{2}}^{1}}=(f, \nabla \varphi)_{\Omega} \quad \text { for all } \varphi \in \dot{W}_{q^{\prime}, \Gamma_{2}}^{1}(\Omega)
$$

This follows from the Hahn-Banach theorem by identifying $\dot{W}_{q^{\prime}, \Gamma_{2}}^{1}(\Omega)$ with a closed subspace of $L^{q^{\prime}}(\Omega)^{d}$ via the mapping $\varphi \mapsto \nabla \varphi$.

We summarize these facts in the following lemma.

Lemma 2 Let $\Omega, q$ be as in Assumption 1. Then for every $F \in \dot{W}_{q, \Gamma_{2}}^{-1}(\Omega)$ and $a \in$ $W_{q}^{1-\frac{1}{q}}(\partial \Omega)$ there is a $p \in \dot{W}_{q, \Gamma_{2}}^{1}(\Omega)$ such that

$$
\begin{aligned}
& (\nabla p, \nabla \varphi)_{\Omega}=\langle F, \varphi\rangle_{\dot{W}_{q, \Gamma_{2}}^{-1}, \dot{W}_{q, \Gamma_{2}}^{1}} \text { for all } \varphi \in \dot{W}_{q^{\prime}, \Gamma_{2}}^{1}(\Omega), \\
& \left.p\right|_{\Gamma_{2}}=a \text { on } \Gamma_{2} \text {. }
\end{aligned}
$$

If $\Gamma_{2} \neq \emptyset, p$ is uniquely determined. If $\Gamma_{2}=\emptyset$, then $p$ is uniquely determined up to a constant. Moreover, there is some constant $C_{q}$ independent of $F$ such that

$$
\|\nabla p\|_{L^{q}(\Omega)^{d}} \leq C_{q}\left(\|F\|_{\dot{W}_{q, \Gamma_{2}}^{-1}(\Omega)}+\|\nabla A\|_{L^{q}(\Omega)}\right)
$$

for all $A \in W_{q}^{1}(\Omega)$ with $\left.A\right|_{\Gamma_{2}}=a$.

Proof First of all, one can easily reduce to the case $a=0$ by extending $a$ to some $A \in$ $W_{q}^{1}(\Omega)$ and considering $p-A$ instead of $p$ and replacing $F$ by $F-(\nabla A, \cdot)_{\Omega}$. Therefore we can assume that $a=0$. Then, as explained above, we find some $f \in L^{q}(\Omega)^{d}$ such that $\langle F, \varphi\rangle=(f, \nabla \varphi)$ for all $\varphi \in \dot{W}_{q^{\prime}, \Gamma_{2}}^{1}(\Omega)$ and $\|f\|_{L^{q}(\Omega)} \leq C\|F\|_{\dot{W}_{q}^{-1}(\Omega)}$. Now $p \in \dot{W}_{q, \Gamma_{2}}^{1}(\Omega)$ solves (2.3), (2.1), resp., if and only if $f=f_{0}+\nabla p$, where $f_{0} \in J_{q}(\Omega)$, i.e., $p$ is determined by the Helmholtz decomposition due to (A2). 


\section{Reduction of the Stokes system}

The aim of this section is to reduce the Stokes system (1.1)-(1.4) for $(v, p)$ to a system only in terms of the velocity $v$ and to eliminate the divergence equation $\operatorname{div} v=g$. The idea goes back to Grubb and Solonnikov, cf. e.g., [39]. ${ }^{1}$ By this reduction the pressure can be treated efficiently even in the case of the boundary condition (1.4) when the pressure enters the boundary condition and therefore cannot be eliminated from the system by applying a Helmholtz projection.

Now we will present the corresponding reduction for the case of general viscosity. Let $v \in W_{q}^{2}(\Omega)^{d}, p \in \dot{W}_{q}^{1}(\Omega)$ with $\left.p\right|_{\Gamma_{2}} \in W_{q}^{1-\frac{1}{q}}\left(\Gamma_{2}\right)$ be a solution of (1.1)-(1.4), where we assume that $f \in L^{q}(\Omega)^{d}, g \in W_{q}^{1}(\Omega)$ with $g \in \dot{W}_{q, \Gamma_{2}}^{-1}(\Omega)$, cf. (2.2), $a \in W_{q}^{1-\frac{1}{q}}\left(\Gamma_{2}\right), \lambda \in \Sigma_{\delta}$, and let $1<q<\infty$ with $q, q^{\prime} \leq \min \left(r_{1}, r_{2}\right)$, where $r_{1}, r_{2}$ and $\Omega$ are as in Assumption 1 .

Now we reduce the Stokes system to a system for $v$ by expressing the pressure $p$ in dependence of $v$ and the data $(f, g, a)$. To this end we multiply (1.1) by an arbitrary $\nabla \varphi$ with $\varphi \in \dot{W}_{q^{\prime}, \Gamma_{2}}^{1}(\Omega)$. Then

$$
(\nabla p, \nabla \varphi)_{\Omega}=(f, \nabla \varphi)_{\Omega}+\lambda\langle g, \varphi\rangle_{\dot{W}_{q, \Gamma_{2}}^{-1}, \dot{W}_{q^{\prime}, \Gamma_{2}}^{1}}+(\operatorname{div}(2 \nu D v), \nabla \varphi)_{\Omega},
$$

where

$$
\begin{aligned}
(\operatorname{div}(2 \nu D v), \nabla \varphi)_{\Omega}= & (v(\Delta v+\nabla \operatorname{div} v), \nabla \varphi)_{\Omega}+(D v, 2 \nabla v \otimes \nabla \varphi)_{\Omega} \\
= & (v(\Delta-\nabla \operatorname{div}) v, \nabla \varphi)_{\Omega} \\
& +(2 \nu \nabla g, \nabla \varphi)_{\Omega}+(D v, 2 \nabla v \otimes \nabla \varphi)_{\Omega} .
\end{aligned}
$$

Hence

$$
\begin{aligned}
(\nabla p, \nabla \varphi)_{\Omega}= & (f, \nabla \varphi)_{\Omega}+\lambda\langle g, \varphi\rangle_{\dot{W}_{q, \Gamma_{2}}^{-1}, \dot{W}_{q^{\prime}, \Gamma_{2}}^{1}(\Omega)}+(2 v \nabla g, \nabla \varphi)_{\Omega} \\
& +(v(\Delta-\nabla \operatorname{div}) v, \nabla \varphi)_{\Omega}+(D v, 2 \nabla v \otimes \nabla \varphi)_{\Omega}
\end{aligned}
$$

for all $\varphi \in \dot{W}_{q^{\prime}, \Gamma_{2}}^{1}(\Omega)$. Now, if $P v \in \dot{W}_{q}^{1}(\Omega)$ with $\left.P v\right|_{\Gamma_{2}} \in W_{p}^{1-\frac{1}{p}}\left(\Gamma_{2}\right)$ is the solution of (1.8)-(1.9), then $p=P v+\tilde{p}$, where $\tilde{p}$ is determined by

$$
\begin{aligned}
(\nabla \tilde{p}, \nabla \varphi)_{\Omega} & =(f, \nabla \varphi)_{\Omega}+\lambda\langle g, \varphi\rangle_{\dot{W}_{q, \Gamma_{2}}^{-1}, \dot{W}_{q^{\prime}, \Gamma_{2}}^{1}(\Omega)}+(2 \nu \nabla g, \nabla \varphi)_{\Omega}, \\
\left.\tilde{p}\right|_{\Gamma_{2}} & =-a_{n}
\end{aligned}
$$

for all $\varphi \in \dot{W}_{q^{\prime}, \Gamma_{2}}^{1}(\Omega)$. Hence $\tilde{p}$ depends only on the data $(f, g, a)$. Here we note that $\tilde{p}$ is uniquely determined by (3.1) (up to a constant if $\Gamma_{2}=\emptyset$ ) due to Lemma 2.

\footnotetext{
${ }^{1}$ In the latter work only the case $\operatorname{div} v=0$ is considered. A corresponding reduction in the general case $\operatorname{div} v=g$ was first presented in [11].
} 
This shows that $v \in W_{q}^{2}(\Omega)^{d}$ solves

$$
\begin{aligned}
\lambda v-\operatorname{div}(v \nabla v)+\nabla P v-\nabla v^{T} \nabla v^{T} & =f_{r} & & \text { in } \Omega, \\
\left.v\right|_{\Gamma_{1}} & =0 & & \text { on } \Gamma_{1}, \\
\left.(n \cdot S(v))_{\tau}\right|_{\Gamma_{2}} & =a_{\tau} & & \text { on } \Gamma_{2}, \\
\left.v \operatorname{div} v\right|_{\Gamma_{2}} & =\left.v g\right|_{\Gamma_{2}} & & \text { on } \Gamma_{2},
\end{aligned}
$$

where

$$
f_{r}=f-\nabla \tilde{p}+v \nabla g
$$

Here we have used that

$$
\operatorname{div}(2 v D v)=\operatorname{div}(v \nabla v)+\nabla v^{T} \nabla v^{T}+v \nabla \operatorname{div} v
$$

We call (3.3)-(3.6) the reduced Stokes system. We note that by the definition of the reduced Stokes operator $A_{q}$, cf. (1.6), $v \in W_{q}^{2}(\Omega)^{d}$ solves (1.1)-(1.4) for some right-hand side $f_{r} \in L^{q}(\Omega)^{d}$ and $a_{\tau}=0,\left.v g\right|_{\Gamma_{2}}=0$ if and only if $v \in \mathcal{D}\left(A_{q}\right)$ and $\left(\lambda+A_{q}\right) v=f_{r}$.

To summarize we have shown:

Lemma 3 Let $f \in L^{q}(\Omega)^{d}, g \in W_{q}^{1}(\Omega) \cap \dot{W}_{q, \Gamma_{2}}^{-1}(\Omega), a \in W_{q}^{1-\frac{1}{q}}\left(\Gamma_{2}\right)$ be given. Then any $v \in W_{q}^{2}(\Omega)^{d}, p \in \dot{W}_{q}^{1}(\Omega)$ with $\left.p\right|_{\Gamma_{2}} \in W_{q}^{1-\frac{1}{q}}\left(\Gamma_{2}\right)$ solving (1.1)-(1.4) is a solution of (3.3)-(3.6) if $f_{r}$ is defined by (3.7) and if $\tilde{p}$ solves (3.1)-(3.2).

Note that in the reduced Stokes system (3.3)-(3.6) the divergence equation div $v=$ $g$ does not appear. Hence, if we want to obtain a solution of the original Stokes system (1.1)-(1.4) by solving the reduced system, it is crucial to prove that $\operatorname{div} v=g$ if the right-hand side is chosen as above. To this end we note that, if $f_{r}$ is defined by (3.7), where $\tilde{p}$ solves (3.1)-(3.2), then $g$ can be derived back from $f_{r}$ because of

$$
-\left(f_{r}, \nabla \varphi\right)_{\Omega}=\lambda\langle g, \varphi\rangle_{\dot{W}_{q, \Gamma_{2}}^{-1}, \dot{W}_{q^{\prime}, \Gamma_{2}}^{1}}+(v \nabla g, \nabla \varphi)_{\Omega}
$$

for all $\varphi \in \dot{W}_{q, \Gamma_{2}}^{1}(\Omega)$. On the other hand, if $v \in W_{q}^{2}(\Omega)^{d}$ solves (3.3)-(3.6), then

$$
-\left(f_{r}, \nabla \varphi\right)_{\Omega}=\lambda\langle\operatorname{div} v, \varphi\rangle_{\dot{W}_{q, \Gamma_{2}}^{-1}, \dot{W}_{q^{\prime}, \Gamma_{2}}^{1}}+(\nu \nabla \operatorname{div} v, \nabla \varphi)_{\Omega}
$$

for all $\varphi \in \dot{W}_{q, \Gamma_{2}}^{1}(\Omega)$ because of (3.3) multiplied with $-\nabla \varphi$ and

$$
\begin{aligned}
& (\operatorname{div}(v \nabla v), \nabla \varphi)_{\Omega}-(\nabla P v, \nabla \varphi)_{\Omega}+\left(\nabla v^{T} \nabla v^{T}, \nabla \varphi\right)_{\Omega} \\
& \quad=(v \Delta v, \nabla \varphi)_{\Omega}-(\nabla P v, \nabla \varphi)_{\Omega}+(D v, 2 \nabla v \otimes \nabla \varphi)_{\Omega}=(v \nabla \operatorname{div} v, \nabla \varphi)_{\Omega}
\end{aligned}
$$

for all $\varphi \in \dot{W}_{q, \Gamma_{2}}^{1}(\Omega)$ due to (1.8). 
In order to conclude $\operatorname{div} v=g$ we need the following assumption.

(A4') Let $\lambda \in \mathbb{C} \backslash(-\infty, 0)$ be such that there is no non-trivial $u \in W_{q, \Gamma_{2}}^{1}(\Omega)$ with

$$
\lambda(u, \varphi)_{\Omega}+(v \nabla u, \nabla \varphi)_{\Omega}=0 \text { for all } \varphi \in W_{q^{\prime}, \Gamma_{2}}^{1}(\Omega) .
$$

Note the assumption (A4) is just (A4') for all $\lambda \in \Sigma_{\delta}$ with $|\lambda| \geq R$ and some $R>0$. As mentioned above it will be shown later that (A1)-(A3) imply (A4) and therefore (A4') for large $\lambda$.

Altogether we obtain:

Lemma 4 Let $f \in L^{q}(\Omega)^{d}, g \in W_{q}^{1}(\Omega) \cap \dot{W}_{q, \Gamma_{2}}^{-1}(\Omega)$ with $\left.g\right|_{\Gamma_{2}} \in W_{q}^{1-\frac{1}{q}}\left(\Gamma_{2}\right)$, $a \in W_{q}^{1-\frac{1}{q}}\left(\Gamma_{2}\right)$ be given and let $f_{r}$ be defined as in (3.7) where $\tilde{p}$ solves (3.1)-(3.2). Moreover, assume that $\left(A 4^{\prime}\right)$ holds. Then any solution $v \in W_{q}^{2}(\Omega)^{d}$ of (3.3)-(3.6) solves (1.1)-(1.4) where $p=P v+\tilde{p} \in \dot{W}_{q}^{1}(\Omega)$ and $\left.p\right|_{\Gamma_{2}} \in W_{q}^{1-\frac{1}{q}}\left(\Gamma_{2}\right)$. Finally, (3.3)(3.6) has no non-trivial solution $v \in W_{q}^{2}(\Omega)^{d}$ with right-hand side $\left(f_{r}, a_{\tau},\left.v g\right|_{\Gamma_{2}}\right)=0$ if and only if (1.1)-(1.4) has no non-trivial solution $v \in W_{q}^{2}(\Omega)^{d}, p \in \dot{W}_{q}^{1}(\Omega)$ with $\left.p\right|_{\Gamma_{2}} \in W_{q}^{1-\frac{1}{q}}\left(\Gamma_{2}\right)$ and right-hand side $(f, g, a)=0$.

Proof If $v$ solves (3.3)-(3.6) with $f_{r}$ as in (3.7) and $\tilde{p}$ solving (3.1)-(3.2), then (3.8)(3.9) imply

$$
\lambda\langle g-\operatorname{div} v, \varphi\rangle_{\dot{W}_{q, \Gamma_{2}}^{-1}, \dot{W}_{q^{\prime}, \Gamma_{2}}^{1}}+(\nu \nabla(g-\operatorname{div} v), \varphi)=0 \text { for all } \varphi \in W_{q^{\prime}, \Gamma_{2}}^{1}(\Omega) .
$$

On the other hand, (3.6) implies $\left.(g-\operatorname{div} v)\right|_{\Gamma_{2}}=0$. Therefore $g-\operatorname{div} v \in W_{q, \Gamma_{2}}^{1}(\Omega)$ and $g-\operatorname{div} v=0$ by $\left(\mathrm{A}^{\prime}\right)$. Thus $v$ solves (1.2). Concerning the boundary condition, using (1.4) it can be easily shown that

$$
\left.p\right|_{\Gamma_{2}}=\left.(n \cdot S(v))_{n}\right|_{\Gamma_{2}}-a_{n},
$$

where $\left.(n \cdot S(v))_{n}\right|_{\Gamma_{2}}$ is equal to $2 v \partial_{n} v_{n}$. Hence (1.4) follows. Altogether we obtain that $(v, p)$ solve (1.1)-(1.4) with $p$ as above.

Finally, assume that (3.3)-(3.6) has no non-trivial solution $v \in W_{q}^{2}(\Omega)^{d}$ with right-hand side $\left(f_{r}, a_{\tau},\left.v g\right|_{\Gamma_{2}}\right)=0$. Moreover, let $v \in W_{q}^{2}(\Omega)^{d}, p \in \dot{W}_{q}^{1}(\Omega)$ with $\left.p\right|_{\Gamma_{2}} \in W_{q}^{1-\frac{1}{q}}\left(\Gamma_{2}\right)$ be a solution of (1.1)-(1.4) with $(f, g, a)=0$. Then $f_{r}=0$ and therefore $v \in W_{q}^{2}(\Omega)^{d}$ solves (3.3)-(3.6) with zero right-hand side due to Lemma 3. Hence $v=0$ by the assumption and therefore the solutions of (1.1)-(1.4) are unique.

Conversely, let $v \in W_{q}^{2}(\Omega)^{d}$ be a solution of (3.3)-(3.6) with right-hand side zero and assume that (1.1)-(1.4) has no non-trivial solution for zero data. Then $\left(f_{r}, \tilde{p}\right)=0$ if $\tilde{p}$ satisfies (3.1)-(3.2) and if $f_{r}$ satisfies (3.7) for $(f, g, a)=0$. Hence $(v, p)$ with $p=P v$ solve (1.1)-(1.4) with $(f, g, a)=0$ by the first part of the lemma. Consequently $v=0$, which proves the converse implication. 


\section{Coordinate transformation}

We start with a simple results on extensions of $\gamma \in W_{r}^{2-\frac{1}{r}}\left(\mathbb{R}^{d-1}\right)$.

Lemma 5 Let $\gamma \in W_{r}^{2-\frac{1}{r}}\left(\mathbb{R}^{d-1}\right), 1<r<\infty$ with $r>d$, and let $\varepsilon>0$. Then there is some $\Gamma \in W_{r}^{2}\left(\mathbb{R}^{d}\right)$ such that $\Gamma\left(x^{\prime}, 0\right)=\gamma\left(x^{\prime}\right), \partial_{x_{d}} \Gamma\left(x^{\prime}, 0\right)=0$ and $\left|\partial_{x_{d}} \Gamma\left(x^{\prime}, x_{d}\right)\right| \leq \varepsilon$ for all $x \in \mathbb{R}^{d}$.

Proof Let $\widetilde{\Gamma} \in W_{r}^{2}\left(\mathbb{R}^{d}\right)$ be an extension of $\gamma \in W_{r}^{2-\frac{1}{r}}\left(\mathbb{R}^{d-1}\right)$ such that $\partial_{x_{d}} \widetilde{\Gamma}\left(x^{\prime}, 0\right)$ $=0$. Then $\Gamma_{\lambda}=\widetilde{\Gamma}\left(x^{\prime}, \lambda x_{d}\right) \in W_{r}^{2}\left(\mathbb{R}^{d}\right)$ is also an extension of $\gamma$ with $\partial_{x_{d}} \Gamma_{\lambda}\left(x^{\prime}, 0\right)=0$ and

$$
\left\|\partial_{x_{d}} \Gamma_{\lambda}\right\|_{L^{\infty}\left(\mathbb{R}^{d}\right)}=|\lambda|\left\|\partial_{x_{d}} \widetilde{\Gamma}\right\|_{L^{\infty}\left(\mathbb{R}^{d}\right)} \rightarrow_{\lambda \rightarrow 0} 0
$$

since $W_{r}^{2}\left(\mathbb{R}^{d}\right) \hookrightarrow C_{b}^{1}\left(\mathbb{R}^{d}\right)$ due to $r>d$. Now we can choose $\lambda>0$ so small that $\Gamma \equiv \Gamma_{\lambda}$ satisfies the statement of the lemma.

The following proposition states the existence of a suitable coordinate transformation, which will lead to a nice structure of the boundary symbol operators of the transformed Stokes system on the half-space. It generalizes a result due to Schumacher [47] and is proved similarly.

Proposition 1 Let $\gamma \in W_{r}^{2-\frac{1}{r}}\left(\mathbb{R}^{d-1}\right)$ with $r>d$. Then there is some $F \in W_{r}^{2}\left(\mathbb{R}^{d}\right)^{d}$ such that $F: \mathbb{R}^{d} \rightarrow \mathbb{R}^{d}$ is a $C^{1}$-diffeomorphism, $F\left(\mathbb{R}_{+}^{d}\right)=\mathbb{R}_{\gamma}^{d}, F\left(x^{\prime}, 0\right)=\left(x^{\prime}, \gamma\left(x^{\prime}\right)\right)$, and $-\left.\partial_{x_{d}} F(x)\right|_{x_{d}=0}=n\left(x^{\prime}, \gamma\left(x^{\prime}\right)\right)$, where $n$ denotes the exterior unit normal of $\partial \mathbb{R}_{\gamma}^{d}$.

Proof The case $r=\infty$ was proved in [47]. Hence it only remains to consider the case $d<r<\infty$. Let $\Gamma \in W_{r}^{2}\left(\mathbb{R}^{d}\right)$ be as in Lemma 5 with $\varepsilon=\frac{1}{2}$. Then we define

$$
F(x)=\left(\begin{array}{c}
x^{\prime} \\
x_{d}+\Gamma(x)
\end{array}\right)-x_{d} k_{D}\left(D_{x}\right) \tilde{n} \equiv \widetilde{F}(x)-x_{d} k_{D}\left(D_{x}\right) \tilde{n}
$$

where $k_{D}\left(D_{x}\right) a=\mathcal{F}_{\xi^{\prime} \mapsto x^{\prime}}^{-1}\left[e^{-\left\langle\xi^{\prime}\right\rangle\left|x_{d}\right|} a^{\prime}\left(\xi^{\prime}\right)\right]$ and

$$
\begin{aligned}
\tilde{n}\left(x^{\prime}\right) & =n\left(x^{\prime}, \gamma\left(x^{\prime}\right)\right)+\left(\partial_{x_{d}} \Gamma\left(x^{\prime}, 0\right)+1\right) e_{d} \\
& =\frac{1}{\sqrt{1+\left|\nabla \gamma\left(x^{\prime}\right)\right|^{2}}}\left(\begin{array}{c}
\nabla \gamma\left(x^{\prime}\right) \\
\sqrt{1+\left|\nabla \gamma\left(x^{\prime}\right)\right|^{2}}-1
\end{array}\right) \in B_{r r}^{1-\frac{1}{r}}\left(\mathbb{R}^{d-1}\right) .
\end{aligned}
$$

Hence $-\partial_{x_{d}} F\left(x^{\prime}, 0\right)=n\left(x^{\prime}, \gamma\left(x^{\prime}\right)\right)$ since $\left.k_{D}\left(D_{x}\right) \tilde{n}\right|_{x_{d}=0}=\tilde{n}$. Furthermore, $\widetilde{F} \in$ $W_{r}^{2}\left(\mathbb{R}^{d}\right)^{d} \hookrightarrow C_{b}^{1}\left(\mathbb{R}^{d}\right)^{d}$ is a diffeomorphism on $\mathbb{R}^{d}$ since $\widetilde{F}\left(x^{\prime}, x_{d}\right)$ is a strictly increasing function in $x_{d}$ for every fixed $x^{\prime} \in \mathbb{R}^{d-1}$. Moreover, $\widetilde{F}$ maps $\mathbb{R}_{+}^{d}$ onto $\mathbb{R}_{\gamma}^{d}$ and $\left\|\nabla \widetilde{F}^{-1}\right\|_{L^{\infty}} \leq 2$ since $\|\nabla \widetilde{F}-I\|_{L^{\infty}} \leq \frac{1}{2}$. We note that $x_{d} k_{D}\left(D_{x}\right)$ is a Poisson operator of order -1 in the sense of Definition 4 below. Hence $f_{ \pm}:=\left.x_{d} k_{D}\left(D_{x}\right) \tilde{n}\right|_{\mathbb{R}_{ \pm}^{d}} \in$ 
$W_{r}^{2}\left(\mathbb{R}_{ \pm}^{d}\right)$ because of Theorem 6 below. Since $\left.f_{+}\right|_{\partial \mathbb{R}_{+}^{d}}=\left.f_{-}\right|_{\partial \mathbb{R}_{+}^{d}}=0$ and $\left.\partial_{x_{d}} f_{+}\right|_{\partial \mathbb{R}_{+}^{d}}=$ $\left.\partial_{x_{d}} f_{-}\right|_{\partial \mathbb{R}_{+}^{d}}=\tilde{n}$, we conclude that $x_{d} k_{D}\left(D_{x}\right) \tilde{n} \in W_{r}^{2}\left(\mathbb{R}^{d}\right)$. Furthermore,

$$
\left\|x_{d} k_{D}\left(D_{x}\right) \tilde{n}\right\|_{C^{1}\left(\mathbb{R}^{d}\right)} \leq C\left\|x_{d} k_{D}\left(D_{x}\right) \tilde{n}\right\|_{W_{r}^{2}\left(\mathbb{R}^{d}\right)} \leq C^{\prime}\left\|\nabla^{\prime} \gamma\right\|_{W_{r}^{1-\frac{1}{r}}\left(\mathbb{R}^{d-1}\right)}
$$

by Theorem 6 again. Hence there is some $\varepsilon>0$ such that

$$
\left\|x_{d} k_{D}\left(D_{x}\right) \tilde{n}\right\|_{C^{1}\left(\mathbb{R}^{d}\right)} \leq \frac{1}{4} \leq \frac{1}{2}\left\|\nabla \widetilde{F}^{-1}\right\|_{\infty}^{-1}
$$

provided that $\|\nabla \gamma\|_{W_{r}^{1-\frac{1}{r}}\left(\mathbb{R}^{d-1}\right)} \leq \varepsilon$. But then

$$
\nabla F(x)=I+\nabla \Gamma \otimes e_{d}-\nabla x_{d} k_{D}\left(D_{x}\right) \tilde{n}
$$

is invertible and

$$
\left\|\nabla F^{-1}\right\|_{\infty} \leq 4 \text { provided that }\left\|\nabla^{\prime} \gamma\right\|_{W_{r}^{1-\frac{1}{r}}\left(\mathbb{R}^{d-1}\right)} \leq \varepsilon .
$$

Moreover, $F: \mathbb{R}^{d} \rightarrow \mathbb{R}^{d}$ is globally invertible since $y=F(x)$ is equivalent to $x=\widetilde{F}^{-1}\left(y+x_{d} k_{D}\left(D_{x}\right) \tilde{n}\right) \equiv H_{y}(x)$ and $H_{y}: \mathbb{R}^{d} \rightarrow \mathbb{R}^{d}$ is a contraction since $\left|\nabla_{x} H_{y}(x)\right| \leq \frac{1}{2}$.

For the general case we consider $\gamma_{\lambda}\left(x^{\prime}\right)=\gamma\left(\lambda x^{\prime}\right), \lambda>0$. Then

$$
\left\|\nabla \gamma_{\lambda}\right\|_{W_{r}^{1-\frac{1}{r}}\left(\mathbb{R}^{d-1}\right)} \leq C\left\|\nabla \gamma_{\lambda}\right\|_{L^{r}\left(\mathbb{R}^{d-1}\right)}^{\frac{1}{r}}\left\|\nabla \gamma_{\lambda}\right\|_{W_{r}^{1}\left(\mathbb{R}^{d-1}\right)}^{1-\frac{1}{2}} \rightarrow \lambda \rightarrow 00
$$

since $r>d$. Hence we can apply the first part and obtain a $C^{1}$-diffeomorphism $F_{\lambda}: \mathbb{R}^{d} \rightarrow \mathbb{R}^{d}$ with respect to $\gamma_{\lambda}$. But then

$$
F=\delta_{\lambda-1} \circ F_{\lambda} \circ \delta_{\lambda} \quad \text { where }\left(\delta_{\lambda} f\right)(x)=f(\lambda x)
$$

is a $C^{1}$-diffeomorphism with the desired properties.

In the following we denote $\left(F^{*} u\right)(x):=u(F(x))$ for $u: \mathbb{R}_{\gamma}^{d} \rightarrow \mathbb{R}$ and $\left(F^{*,-1} v\right)(x)$ $:=v\left(F^{-1}(x)\right)$ for $v: \mathbb{R}_{+}^{d} \rightarrow \mathbb{R}$, where $F$ is as in the latter proposition.

Corollary 1 Let $\gamma \in W_{r}^{2-\frac{1}{r}}\left(\mathbb{R}^{d-1}\right)$ with $r>d$. Then

$$
\begin{aligned}
& F^{*}: W_{q}^{1}\left(\mathbb{R}_{\gamma}^{d}\right) \rightarrow W_{q}^{1}\left(\mathbb{R}_{+}^{d}\right), F^{*}: \dot{W}_{q}^{1}\left(\mathbb{R}_{\gamma}^{d}\right) \rightarrow \dot{W}_{q}^{1}\left(\mathbb{R}_{+}^{d}\right) \text { for all } 1 \leq q \leq \infty, \\
& F^{*}: W_{q}^{2}\left(\mathbb{R}_{\gamma}^{d}\right) \rightarrow W_{q}^{2}\left(\mathbb{R}_{+}^{d}\right) \text { for all } 1 \leq q \leq r
\end{aligned}
$$

continuously. Moreover, the corresponding statements are true for $F^{*,-1}$. Finally, if $\left(F_{0}^{*} a\right)\left(x^{\prime}\right)=a\left(x^{\prime}, \gamma\left(x^{\prime}\right)\right)$ for $a \in C_{b}^{1}\left(\partial \mathbb{R}_{\gamma}^{d}\right)$ and $\left(F_{0}^{*,-1} a\right)(x)=a\left(\left.F^{-1}(x)\right|_{\partial \mathbb{R}_{\gamma}^{d}}\right)$ for 
$a \in C_{b}^{1}\left(\mathbb{R}^{d-1}\right)$, then

$$
F_{0}^{*}: W_{q}^{s}\left(\partial \mathbb{R}_{\gamma}^{d}\right) \rightarrow W_{q}^{s}\left(\mathbb{R}^{d-1}\right)
$$

is a bounded mapping for all $1<q<\infty, 0 \leq s<1$, with continuous inverse $F_{0}^{*,-1}$.

Proof The first statements easily follow from the chain and product rule, where we note that

$$
\nabla\left(F^{*} u\right)=\nabla F(x)(\nabla u)(F(x))
$$

where $\nabla F \in W_{r}^{1}\left(\mathbb{R}_{+}^{d}\right)$ and $(\nabla u)(F(x)) \in W_{q}^{1}\left(\mathbb{R}_{+}^{d}\right)$ if $u \in W_{q}^{2}(\Omega)$. Therefore $\nabla F F^{*}(\nabla u) \in W_{q}^{1}\left(\mathbb{R}_{+}^{d}\right)$ for all $1 \leq q \leq r$ due to Lemma 1 .

For the last statement we note that $W_{q}^{s}\left(\partial \mathbb{R}_{\gamma}^{d}\right)$ is normed by

$$
\|a\|_{W_{q}^{s}\left(\partial \mathbb{R}_{\gamma}^{d}\right)}^{q}=\|a\|_{L^{q}\left(\partial \mathbb{R}_{\gamma}^{d}\right)}^{q}+\int_{L^{q}\left(\partial \mathbb{R}_{\gamma}^{d}\right)} \int_{L^{q}\left(\partial \mathbb{R}_{\gamma}^{d}\right)} \frac{|a(x)-a(y)|^{q}}{|x-y|^{d-1+s q}} d \sigma(x) d \sigma(y),
$$

where $d \sigma$ denotes integration with respect to the surface measure on $\partial \mathbb{R}_{\gamma}^{d}$. Since $F_{0} \equiv$ $\left.F\right|_{\mathbb{R}^{d-1}}: \mathbb{R}^{d-1} \rightarrow \partial \mathbb{R}_{\gamma}^{d}$ is a $C^{1}$-diffeomorphism, $\left\|F_{0}^{*} a\right\|_{L^{q}\left(\mathbb{R}^{d-1}\right)} \leq C\|a\|_{L^{q}\left(\partial \mathbb{R}_{\gamma}^{d}\right)}$ and

$$
\begin{aligned}
& \int_{\mathbb{R}^{d-1}} \int_{\mathbb{R}^{d-1}} \frac{\left|a\left(F_{0}\left(x^{\prime}\right)\right)-a\left(F_{0}\left(y^{\prime}\right)\right)\right|^{q}}{\left|x^{\prime}-y^{\prime}\right|^{d-1+s q}} d x^{\prime} d y^{\prime} \\
& \leq C \int_{\mathbb{R}^{d-1}} \int_{\mathbb{R}^{d-1}} \frac{\left|a\left(F_{0}\left(x^{\prime}\right)\right)-a\left(F_{0}\left(y^{\prime}\right)\right)\right|^{q}}{\left|F_{0}\left(x^{\prime}\right)-F_{0}\left(y^{\prime}\right)\right|^{d-1+s q}} J\left(x^{\prime}\right) J\left(y^{\prime}\right) d x^{\prime} d y^{\prime} \\
& =C \int_{\partial \mathbb{R}_{\gamma}^{d}} \int_{\partial \mathbb{R}_{\gamma}^{d}} \frac{|a(x)-a(y)|^{q}}{\left|F_{0}^{-1}(x)-F_{0}^{-1}(y)\right|^{d-1+s q}} d \sigma(x) d \sigma(y) \leq C\|a\|_{B_{q q}^{s}\left(\partial \mathbb{R}_{\gamma}^{d}\right)}^{q}
\end{aligned}
$$

where $J\left(z^{\prime}\right)=\operatorname{det}\left(\nabla F_{0}\left(z^{\prime}\right)^{T} \nabla F_{0}\left(z^{\prime}\right)\right)^{\frac{1}{2}}$. Hence $F_{0}^{*}: W_{q}^{s}\left(\partial \mathbb{R}_{\gamma}^{d}\right) \rightarrow W_{q}^{s}\left(\mathbb{R}^{d-1}\right)$ is continuous. The statement for $F_{0}^{*,-1}$ is proved in the same way.

Corollary 2 Let $d<r_{2} \leq \infty, 1 \leq q<\infty$, and let $\Omega \subseteq \mathbb{R}^{d}, d \geq 2$, be a domain satisfying the assumption (A1). Then there are linear bounded operators $E_{0}: W_{q}^{1-\frac{1}{q}}(\partial \Omega) \rightarrow W_{q}^{1}(\Omega)$ and $E_{1}: W_{q}^{2-\frac{1}{q}}(\partial \Omega) \times W_{q}^{1-\frac{1}{q}}(\partial \Omega) \rightarrow W_{q}^{2}(\Omega)$ if $1<q \leq r$ such that

$$
\gamma_{0} E_{0} a=a \text { and }\left(\begin{array}{l}
\gamma_{0} \\
\gamma_{1}
\end{array}\right) E_{1} b=b
$$

for all $a \in W_{q}^{1-\frac{1}{q}}(\partial \Omega), b \in W_{q}^{2-\frac{1}{q}}(\partial \Omega) \times W_{q}^{1-\frac{1}{q}}(\partial \Omega)$. 
Proof First let $\Omega=\mathbb{R}_{\gamma}^{d}$ with $\gamma \in W_{r_{2}}^{2-\frac{1}{r_{2}}}\left(\mathbb{R}^{d-1}\right)$. Using Proposition 1 and Corollary 1 , the statement is easily reduced to the corresponding statements for a halfspace $\mathbb{R}_{+}^{d}$, where we note that $-\left.\partial_{d} F^{*} v\right|_{\mathbb{R}^{d-1}}=\left.F_{0}^{*} \partial_{n} v\right|_{\partial \mathbb{R}_{\gamma}^{d}}=F_{0}^{*} \gamma_{1} v$ for all $v \in$ $C_{(0)}^{1}\left(\overline{\mathbb{R}}_{\gamma}^{d}\right)$.

If $\Omega$ is a general domain satisfying the assumption (A1), then the statement for $E_{0}$ is easily reduced to the case of finitely many bent half-spaces $\mathbb{R}_{\gamma_{j}}^{d}$ using the partition of unity assumed in (A1). The extension operator $E_{1} b$ can be constructed as follows: Let $v \in W_{q}^{2}(\Omega)$ be such that $\left.v\right|_{\partial \Omega}=b_{1}$, where $b=\left(b_{1}, b_{2}\right)$. Moreover, let $w_{j} \in W_{q}^{2}\left(\mathbb{R}_{\gamma_{j}}^{d}\right)$ be such that $\left.w_{j}\right|_{\partial \mathbb{R}_{\gamma_{j}}^{d}}=0$ and $\left.\partial_{n} w_{j}\right|_{\partial \mathbb{R}_{\gamma_{j}}^{d}}=\psi_{j} b_{2}-\left.\psi_{j} \partial_{n} v\right|_{\partial \mathbb{R}_{\gamma_{j}}^{d}}$. Then $w=\sum_{j=1}^{N} \varphi_{j} w_{j}$ satisfies $\left.w\right|_{\partial \Omega}=0$ and $\left.\partial_{n} w\right|_{\partial \Omega}=b_{2}-\left.\partial_{n} v\right|_{\partial \Omega}$. Therefore $E_{1} b:=v+w$ has the desired properties. Obviously, the extension operators can be constructed to become bounded operators.

In the following we will denote the variables and operators corresponding to the original problem in $\mathbb{R}_{\gamma}^{d}$ by $x, \xi, \nabla, \ldots$ and of the transformed problem in $\mathbb{R}_{+}^{d}$ by $\underline{x}, \underline{\xi}, \underline{\nabla}, \ldots$. Similarly, $\underline{a}\left(\underline{x}^{\prime}, \xi\right)$ will indicate the symbols of the transformed problem and $a(\xi)$ the symbols of the model operator (the corresponding operator on $\left.\mathbb{R}_{+}^{d}\right)$.

In the following, let $U=U\left(\underline{x}^{\prime}\right)$ be an orthonormal matrix which maps the exterior normal vector

$$
\underline{n}\left(\underline{x}^{\prime}\right)=\frac{1}{\sqrt{1+\left|\nabla^{\prime} \gamma\left(\underline{x}^{\prime}\right)\right|^{2}}}\left(\begin{array}{c}
\nabla^{\prime} \gamma\left(\underline{x}^{\prime}\right) \\
-1
\end{array}\right)
$$

on $\partial \mathbb{R}_{\gamma}^{d}$ at the point $\left(\underline{x}^{\prime}, \gamma\left(\underline{x}^{\prime}\right)\right)$ to $-e_{d}$, which is the exterior normal on $\mathbb{R}_{+}^{d}$.

Using this notation,

$$
\nabla F^{*,-1} v=F^{*,-1} U^{T}\left(\underline{x}^{\prime}\right) A(\underline{x}) \nabla v=F^{*,-1} \mathrm{OP}\left(U^{T}\left(\underline{x}^{\prime}\right) A(\underline{x}) i \underline{\xi}\right) v,
$$

where $A(\underline{x}) \underline{\xi}=U\left(\underline{x}^{\prime}\right)\left(\nabla_{\underline{x}} F(\underline{x})\right)^{-1} \underline{\xi}$ and $v \in C^{1}\left(\overline{\mathbb{R}}_{\gamma}^{d}\right)$. Then $\left(\left.A\right|_{x_{d}=0}\right)^{-T}$ has the structure

$$
A\left(\underline{x}^{\prime}, 0\right)^{-T}=U\left(\underline{x}^{\prime}\right)\left(\begin{array}{cc}
I^{\prime} & -\underline{n}^{\prime}\left(\underline{x}^{\prime}\right) \\
\nabla^{\prime} \gamma\left(\underline{x}^{\prime}\right)^{T} & -\underline{n}_{d}\left(\underline{x}^{\prime}\right)
\end{array}\right)=\left(\begin{array}{cc}
A^{\prime}\left(\underline{x}^{\prime}\right)^{-T} & 0 \\
0 & 1
\end{array}\right)
$$

due to Proposition 1 , where $A^{\prime}\left(\underline{x}^{\prime}, 0\right)$ depends smoothly on $\nabla^{\prime} \gamma\left(\underline{x}^{\prime}\right)$. Hence $\left.A\right|_{x_{d}=0}$ has the same structure with $A^{\prime}\left(\underline{x}^{\prime}, 0\right)^{-T}$ replaced by $A^{\prime}\left(\underline{x}^{\prime}, 0\right)$.

Remark 2 Note that relation (4.1) is of much simpler structure than the corresponding relation in the previous work [11, Equation (5.15)]. This leads to some simplifications in the present proofs. The more complicated structure in [11] was due to the simple coordinate transformation $\widetilde{F}(x)=\left(\begin{array}{c}x^{\prime} \\ x_{d}+\gamma\left(x^{\prime}\right)\end{array}\right)$, which was used in order to deal 
with a boundary of regularity $C^{1,1}$. The coordinate transformation due to Proposition 1 admits to work with $C^{1,1}$-boundary again (if $r_{2}=\infty$ ). But it has the same structural properties as the coordinate transformation used in [32,33], i.e., that normal directions are preserved at the boundary, which leads to (4.1). Note that, if one would apply directly the coordinate transformation used in [32,33], one would need higher regularity assumptions on $\partial \Omega$, e.g., $C^{2,1}$ instead of $C^{1,1}$.

In the following we will for simplicity write $A\left(\underline{x}^{\prime}\right)$ instead of $A\left(\left(\underline{x}^{\prime}, 0\right)\right)$. Moreover, we denote $\gamma_{j} u=\left.\left(-\partial_{x_{d}}\right)^{j} u\right|_{\partial \mathbb{R}_{+}^{d}}$ and $\gamma_{n} v=n \cdot \gamma_{0} v$. More generally, the transformed differential and trace operators needed in the following are considered in the next lemma.

Lemma 6 Let $v \in C_{(0)}^{\infty}\left(\overline{\mathbb{R}}_{\gamma}^{d}\right), u \in C_{(0)}^{\infty}\left(\overline{\mathbb{R}}_{\gamma}^{d}\right)^{d}$, and let $F$ be as in Proposition 1. Then

$$
\begin{aligned}
& F^{*} \nabla v=\underline{\nabla} F^{*} v, \quad F^{*} \operatorname{div} u=\underline{\operatorname{div}} F^{*} u, \quad F^{*} \Delta u=\underline{\Delta} F^{*} u+R_{1} F^{*} u, \\
& F_{0}^{*} \gamma_{n} u=\underline{\gamma_{n}} F^{*} u, \quad F_{0}^{*} \gamma_{1} v=\underline{\gamma_{1}} F^{*} v, \quad F_{0}^{*} T_{1}^{\prime} u=\underline{t_{1}^{\prime}}\left(\underline{x^{\prime}}, D_{x}\right) F^{*} u,
\end{aligned}
$$

where

1.

$$
\begin{aligned}
& \underline{\nabla}=\mathrm{OP}\left(U^{T}\left(\underline{x}^{\prime}\right) A(\underline{x}) i \underline{\xi}\right), \quad \underline{\operatorname{div}} u=\mathrm{OP}\left((A(\underline{x}) i \underline{\xi})^{T} U\left(\underline{x}^{\prime}\right)\right) u, \\
& \underline{\Delta}=-\mathrm{OP}\left(|A(\underline{x}) \underline{\xi}|^{2}\right), \quad \underline{\gamma_{n}}=-e_{d} \cdot \gamma_{0} U\left(\underline{x}^{\prime}\right), \\
& \underline{\gamma_{1}}=\underline{\gamma_{n}} \underline{\nabla}=-\gamma_{0} \partial_{d} .
\end{aligned}
$$

2. $R_{1}$ is a differential operator of order 1 with $L^{r_{2}}$-coefficients, $r_{2}>d$.

3. $\underline{t}_{1}^{\prime}\left(\underline{x}^{\prime}, D_{x}\right) u=-\gamma_{0} U^{T}\left(\underline{x}^{\prime}\right) O P\left(\begin{array}{cc}i \underline{\xi}_{d} I^{\prime} & A^{\prime}\left(\underline{x}^{\prime}\right) i \underline{\xi^{\prime}} \\ \left(A^{\prime}\left(\underline{x}^{\prime}\right) i \underline{\xi}^{\prime}\right)^{T} & i \underline{\xi}_{d}\end{array}\right) U\left(\underline{x}^{\prime}\right) u$.

If additionally $\gamma_{0} u=0$, then

$$
F_{0}^{*} \gamma_{n}(\Delta-\nabla \operatorname{div}) u=\underline{t}_{0}\left(\underline{x}^{\prime}, D_{x}\right) F^{*} u
$$

where $\underline{t}_{0}\left(\underline{x}^{\prime}, D_{x}\right)=\mathrm{OP}^{\prime}\left(\left(A^{\prime}\left(\underline{x}^{\prime}\right) i \xi^{\prime}\right)^{T}\left(U\left(\underline{x}^{\prime}\right) \gamma_{1}\right)\right)$.

Proof The proof is done in the same way as in [11, Lemma 5.6] except for the last statement. In order to prove the last statement, we use the identity

$$
\left.n \cdot(\Delta-\nabla \operatorname{div}) u\right|_{\partial \mathbb{R}_{\gamma}^{d}}=\left.\operatorname{div}_{\tau} \partial_{n} u\right|_{\partial \mathbb{R}_{\gamma}^{d}} \text { if } \gamma_{0} u=0
$$

Here $\operatorname{div}_{\tau} w=\left.\operatorname{Tr}\left(P_{\tau} \nabla W\right)\right|_{\partial \mathbb{R}_{\gamma}^{d}}$ for all $w \in C^{1}\left(\partial \mathbb{R}_{\gamma}^{d}\right)^{d}$ where $W \in C^{1}\left(\overline{\mathbb{R}_{\gamma}^{d}}\right)^{d}$ is an arbitrary extension of $w$ and $P_{\tau}=P_{\tau}(x)$ denotes the orthogonal projection onto the tangent space of $\partial \mathbb{R}_{\gamma}^{d}$ at $x \in \partial \mathbb{R}_{\gamma}^{d}$. It is easy to check that 


$$
F_{0}^{*}\left(P_{\tau}\right)\left(\underline{x}^{\prime}\right)=U^{T}\left(\underline{x}^{\prime}\right)\left(I-e_{d} \otimes e_{d}\right) U\left(\underline{x}^{\prime}\right) .
$$

Hence

$$
\begin{aligned}
F_{0}^{*}\left(\operatorname{div}_{\tau} w\right) & =\left.\operatorname{Tr}\left(U^{T}\left(\underline{x}^{\prime}\right)\left(I-e_{d} \otimes e_{d}\right) U\left(\underline{x}^{\prime}\right) U^{T}\left(\underline{x}^{\prime}\right) A\left(\underline{x}^{\prime}\right) \nabla F^{*} w\right)\right|_{x_{d}=0} \\
& =\left.\operatorname{Tr}\left(U^{T}\left(\underline{x}^{\prime}\right)\left(I-e_{d} \otimes e_{d}\right) A\left(\underline{x}^{\prime}\right) \nabla F^{*} w\right)\right|_{x_{d}=0} \\
& =\left.\mathcal{F}_{\xi \mapsto x}^{-1}\left[\operatorname{Tr}\left(U^{T}\left(\underline{x}^{\prime}\right)\left(I-e_{d} \otimes e_{d}\right) A\left(\underline{x}^{\prime}\right) i \xi \otimes \hat{v}(\xi)\right)\right]\right|_{x_{d}=0} \\
& =\mathcal{F}_{\xi^{\prime} \mapsto x^{\prime}}^{-1}\left[\left(A^{\prime}\left(\underline{x}^{\prime}\right) i \xi^{\prime}\right)^{T}\left(U\left(\underline{x}^{\prime}\right) \hat{v}\left(\xi^{\prime}, 0\right)\right)^{\prime}\right]
\end{aligned}
$$

where $v=F^{*} w$. From this identity the statement follows because of $F_{0}^{*}\left(\left.\partial_{n} u\right|_{\partial \mathbb{R}_{\gamma}^{d}}\right)=$ $\gamma_{1} F_{0}^{*} u$.

Lemma 7 Let $d<r_{2} \leq \infty, 1<q \leq r_{2}, j=0,1, \lambda \in \mathbb{C}$, and let $\Omega \subseteq \mathbb{R}^{d}, d \geq 2$, be a domain satisfying the assumption (A1). Then there is a continuous extension operator $E_{j}: W_{q}^{2-j-\frac{1}{q}}(\Omega) \rightarrow W_{q}^{2}(\Omega)$ such that

$$
\begin{aligned}
& \langle\lambda\rangle\left\|E_{j} a\right\|_{L^{q}(\Omega)}+\left\|\nabla^{2} E_{j} a\right\|_{L^{q}(\Omega)} \\
& \leq C\left(\langle\lambda\rangle^{1-\frac{j}{2}-\frac{1}{2 q}}\|a\|_{L^{q}(\partial \Omega)}+\|a\|_{W_{q}^{2-j-\frac{1}{q}}(\partial \Omega)}\right)
\end{aligned}
$$

for $j=0,1$ and $T_{1}^{\prime} E_{1} a=a$ as well as $\left.E_{0} a\right|_{\partial \Omega}=a$, where $T_{1}^{\prime}$ is defined as in (1.7).

Proof First let $j=0$ and let $\Omega=\mathbb{R}_{\gamma}^{d}, \gamma \in W_{r_{2}}^{2-\frac{1}{r_{2}}}\left(\mathbb{R}^{d-1}\right)$. Using the coordinate transformation due to Proposition 1 , the statement is easily reduced to the case of a half-space $\mathbb{R}_{+}^{d}$. In the latter case the statement can be reduced to the case $\lambda=1$ by the same scaling argument as in [38, Sect. 1.1]. If $j=0$ and $\Omega$ is a general domain satisfying the assumption (A1), then one can prove the statement easily with the aid of the partition of unity and the statement for a bent half space.

Next let $j=1$. Then we choose $E_{1} a \in W_{q}^{2}(\Omega)$ such that $\left.E_{1} a\right|_{\partial \Omega}=0$ and $\left.\partial_{n} E_{1} a\right|_{\partial \Omega}=v^{-1} a$. By the same arguments as in the case $j=0$ one can choose $E_{1} a$ such that (4.2) holds. (Again one reduces to the case of a half-space and uses a simple scaling argument). Then

$$
\begin{aligned}
& \left(T_{1}^{\prime} E_{1} a\right)_{\tau}=\left.v\left(\left(\partial_{n} E_{1} a\right)_{\tau}+\nabla_{\tau} E_{1} a_{n}\right)\right|_{\partial \Omega}=a_{\tau}+0 \\
& \left(T_{1}^{\prime} E_{1} a\right)_{n}=\left.v\left(\operatorname{div}\left((I-n \otimes n) E_{1} a\right)+\left(\partial_{n} E_{1} a\right)_{n}\right)\right|_{\partial \Omega}=0+a_{n}
\end{aligned}
$$

since $\left.E_{1} a\right|_{\partial \Omega}=0$. Hence $T_{1}^{\prime} E_{1} a=a$. 


\section{Construction of the approximative resolvent}

The proof of Theorem 1 is based on the following result.

Theorem 4 Let $\mathbb{R}_{\gamma}^{d}, d \geq 2, \gamma \in W_{r_{2}}^{2-\frac{1}{r} 2}\left(\mathbb{R}^{d-1}\right)$, be a bent half-space, let $v, q, r, r_{2}, \tau$ be as in Assumption $1, j=0,1$, and let $\delta \in(0, \pi)$. Then there are bounded operators $R_{j, \lambda}: L^{q}\left(\mathbb{R}_{\gamma}^{d}\right)^{d} \rightarrow W_{q}^{2}\left(\mathbb{R}_{\gamma}^{d}\right)^{d}, G_{j, \lambda}: L^{q}\left(\mathbb{R}_{\gamma}^{d}\right)^{d} \rightarrow W_{q}^{1}\left(\mathbb{R}_{\gamma}^{d}\right)^{d}$ such that

$$
\begin{aligned}
& (\lambda-\operatorname{div}(v \nabla \cdot)) R_{j, \lambda} f+\nabla G_{j, \lambda} f=f+S_{j, \lambda} f \text { in } \mathbb{R}_{\gamma}^{d}, \\
& \left.R_{0, \lambda} f\right|_{\partial \mathbb{R}_{\gamma}^{d}}=0 \quad \text { on } \partial \mathbb{R}_{\gamma}^{d} \text { if } j=0 \text {, } \\
& T_{1}^{\prime} R_{1, \lambda} f=0 \quad \text { on } \partial \mathbb{R}_{\gamma}^{d} \text { if } j=1,
\end{aligned}
$$

for every $f \in L^{q}\left(\mathbb{R}_{\gamma}^{d}\right)^{d}$ and $\lambda \in \mathbb{C} \backslash(-\infty, 0]$ as well as

$$
\left(\nabla G_{j, \lambda} f, \nabla \varphi\right)_{\mathbb{R}_{\gamma}^{d}}=\left(\nu(\Delta-\nabla \operatorname{div}) R_{j, \lambda} f, \nabla \varphi\right)_{\mathbb{R}_{\gamma}^{d}}+\left\langle S_{j, \lambda}^{\prime} f, \varphi\right\rangle_{W_{q, 0}^{-1}, W_{q^{\prime}}^{1}}
$$

for all $\varphi \in W_{q^{\prime}}^{1}\left(\mathbb{R}_{\gamma}^{d}\right)$ with $\left.\varphi\right|_{\partial \mathbb{R}_{\gamma}^{d}}=0$ if $j=1$ and

$$
\left.G_{1, \lambda} f\right|_{\partial \mathbb{R}_{\gamma}^{d}}=\left.2 \nu\left(\partial_{n} R_{1, \lambda} f\right)_{n}\right|_{\partial \mathbb{R}_{\gamma}^{d}}+S_{\lambda}^{\prime \prime} f \quad \text { on } \partial \mathbb{R}_{\gamma}^{d}
$$

where

$$
\begin{aligned}
& \left\|S_{j, \lambda}\right\|_{\mathcal{L}\left(L^{q}\left(\mathbb{R}_{\gamma}^{d}\right)\right)}+\left\|S_{0, \lambda}^{\prime}\right\|_{\mathcal{L}\left(L^{q}\left(\mathbb{R}_{\gamma}^{d}\right), W_{q, 0}^{-1}\left(\mathbb{R}_{\gamma}^{d}\right)\right)} \leq C_{q, \delta}\langle\lambda\rangle^{-\varepsilon}, \\
& \left\|S_{1, \lambda}^{\prime}\right\|_{\mathcal{L}\left(L^{q}\left(\mathbb{R}_{\gamma}^{d}\right), W_{q}^{-1}\left(\mathbb{R}_{\gamma}^{d}\right)\right)}+\left\|S_{\lambda}^{\prime \prime}\right\|_{\mathcal{L}\left(L^{q}\left(\mathbb{R}_{\gamma}^{d}\right), W_{q}{ }^{1-\frac{1}{q}}\left(\partial \mathbb{R}_{\gamma}^{d}\right)\right)} \leq C_{q, \delta}\langle\lambda\rangle^{-\varepsilon}
\end{aligned}
$$

uniformly in $\lambda \in \Sigma_{\delta}$ for some $\varepsilon>0$. Moreover,

$$
\begin{gathered}
(1+|\lambda|)\left\|R_{j, \lambda}\right\|_{\mathcal{L}\left(L^{q}\left(\mathbb{R}_{\gamma}^{d}\right)\right)}+\left\|\nabla^{2} R_{j, \lambda}\right\|_{\mathcal{L}\left(L^{q}\left(\mathbb{R}_{\gamma}^{d}\right)\right)} \leq C_{q, \delta}, \\
\left(1+|\lambda|^{\frac{1}{2}}\right)\left\|G_{j, \lambda}\right\|_{\mathcal{L}\left(L^{q}\left(\mathbb{R}_{\gamma}^{d}\right)\right)}+\left\|\nabla G_{j, \lambda}\right\|_{\mathcal{L}\left(L^{q}\left(\mathbb{R}_{\gamma}^{d}\right)\right)} \leq C_{q, \delta}, \\
\left\|\int_{\Gamma_{R}} h(-\lambda) R_{j, \lambda} d \lambda\right\|_{\mathcal{L}_{\left(L^{q}\left(\mathbb{R}_{\gamma}^{d}\right)\right)}} \leq C_{q, \delta}\|h\|_{\infty},
\end{gathered}
$$

uniformly in $\lambda \in \Sigma_{\delta}$ and $h \in H(\delta)$.

Remark 3 Here the operator $G_{j, \lambda}$ represents the principal part of $P R_{j, \lambda}$, cf. (1.8)(1.9) and note that the term $\nabla v^{T} D v$ is of lower order compared to $v(\Delta-\nabla \operatorname{div}) v$. Lower order terms in general will give rise to a contribution to the remainder terms $S_{j, \lambda}, S_{j, \lambda}^{\prime}$, and $S_{\lambda}^{\prime \prime}$.

The theorem will be proved with aid of the calculus of pseudodifferential boundary value problems with non-smooth coefficient as developed in $[9,11]$. 
5.1 Pseudodifferential operators with non-smooth coefficients

In the following we denote $D_{x_{j}}=\frac{1}{i} \partial_{x_{j}}$ and $D_{x}=\left(D_{x_{1}}, \ldots, D_{x_{d}}\right)$.

Definition 1 Let $X$ be a Banach space and let $\tau>0$. Then the symbol space $\mathcal{C}^{\tau} S_{1,0}^{m}\left(\mathbb{R}^{d} \times \mathbb{R}^{d} ; X\right), m \in \mathbb{R}$, is the set of all functions $p: \mathbb{R}^{d} \times \mathbb{R}^{d} \rightarrow X$ that are smooth with respect to $\xi$ and are in $\mathcal{C}^{\tau}\left(\mathbb{R}^{d}\right)$ with respect to $x$ satisfying

$$
\left\|D_{\xi}^{\alpha} p(., \xi)\right\|_{\mathcal{C}^{\tau}\left(\mathbb{R}^{d} ; X\right)} \leq C_{\alpha}\langle\xi\rangle^{m-|\alpha|}
$$

for all $\alpha \in \mathbb{N}_{0}^{d}$. Moreover, we define for $k \in \mathbb{N}$ the semi-norm

$$
|p|_{k}^{(m)}:=\sup _{|\alpha| \leq k, \xi \in \mathbb{R}^{d}}\langle\xi\rangle^{|\alpha|-m}\left\|D_{\xi}^{\alpha} p(., \xi)\right\|_{\mathcal{C}^{\tau}\left(\mathbb{R}^{d} ; X\right)}
$$

Finally, $C^{\tau} S_{1,0}^{m}\left(\mathbb{R}^{d} \times \mathbb{R}^{d} ; X\right)$ denotes the corresponding space with $\mathcal{C}^{\tau}$ replaced by $C^{\tau}$.

Given $p \in \mathcal{C}^{\tau} S_{1,0}^{m}\left(\mathbb{R}^{d} \times \mathbb{R}^{d} ; \mathcal{L}\left(X_{0}, X_{1}\right)\right)$, where $X_{0}, X_{1}$ are two Banach spaces, we define

$$
\begin{aligned}
& p\left(x, D_{x}\right) u \equiv \operatorname{OP}(p(x, \xi)) u=\int_{\mathbb{R}^{d}} e^{i x \cdot \xi} p(x, \xi) \hat{u}(\xi) d \xi \text { and } \\
& p\left(D_{x}, x\right) u \equiv \operatorname{OP}(p(y, \xi)) u=\int_{\mathbb{R}^{d}} \int_{\mathbb{R}^{d}} e^{i(x-y) \cdot \xi} p(y, \xi) u(y) d y d \xi
\end{aligned}
$$

for $u \in \mathcal{S}\left(\mathbb{R}^{d} ; X_{0}\right)$ are the associated pseudodifferential operators in $L$ - and $R$-form, respectively; also called $x$-form and $y$-form. Here the second integral has to be understood as iterated integral or oscillatory integral, cf. [41, Theorem 2.2]. If $p \in$ $\mathcal{C}^{\tau} S_{1,0}^{m}\left(\mathbb{R}^{d-1} \times \mathbb{R}^{d-1} ; \mathcal{L}\left(X_{0}, X_{1}\right)\right)$, then $p\left(x^{\prime}, D_{x^{\prime}}\right)=\mathrm{OP}^{\prime}\left(p\left(x^{\prime}, \xi^{\prime}\right)\right)$ and $p\left(D_{x^{\prime}}, x^{\prime}\right)=$ $\mathrm{OP}^{\prime}\left(p\left(y^{\prime}, \xi^{\prime}\right)\right)$ denote the corresponding pseudodifferential operators acting on functions defined on $\mathbb{R}^{d-1}$.

Concerning boundedness on Bessel potential spaces, we recall

Theorem 5 Let $\tau>0,1<q<\infty, m \in \mathbb{R}$, and let $H_{0}, H_{1}$ be Hilbert spaces. If $p \in \mathcal{C}^{\tau} S_{1,0}^{m}\left(\mathbb{R}^{d} \times \mathbb{R}^{d} ; \mathcal{L}\left(H_{0}, H_{1}\right)\right)$ and $s \in(-\tau, \tau)$, then $p\left(x, D_{x}\right)$ and $p\left(D_{x}, x\right)$ extend to bounded linear operators

$$
\begin{aligned}
& p\left(x, D_{x}\right): H_{q}^{s+m}\left(\mathbb{R}^{d} ; H_{0}\right) \rightarrow H_{q}^{s}\left(\mathbb{R}^{d} ; H_{1}\right) \quad \text { and } \\
& p\left(D_{x}, x\right): H_{q}^{s}\left(\mathbb{R}^{d} ; H_{0}\right) \rightarrow H_{q}^{s-m}\left(\mathbb{R}^{d} ; H_{1}\right) .
\end{aligned}
$$

Moreover, the operators depend continuously on the symbols with respect to the operator norm and the symbol semi-norms. 
We refer to [11, Theorem 3.2] for references and comments on the proof. The continuous dependence is not stated explicitly there; but this follows from linearity of the mapping $p \mapsto\left(p\left(x, D_{x}\right), p\left(D_{x}, x\right)\right)$ and the fact that the operator norms can be bounded in terms of the symbol semi-norms only.

Note that the latter theorem is also true for $p \in C^{\tau} S_{1,0}^{m}\left(\mathbb{R}^{d} \times \mathbb{R}^{d} ; \mathcal{L}\left(H_{0}, H_{1}\right)\right)$ since $C^{\tau}\left(\mathbb{R}^{d} ; X\right)=\mathcal{C}^{\tau}\left(\mathbb{R}^{d} ; X\right)$ for $\tau \notin \mathbb{N}$ and $(-\tau, \tau)$ is an open interval. (Hence the result for $\tau \in \mathbb{N}$ follows from the result for $\tau^{\prime} \notin \mathbb{N}$ with $|s|<\tau^{\prime}<\tau$ ).

In order to deal with the low regularity of $\nu \in W_{r_{1}}^{1}(\Omega)$ and $\gamma \in W_{r_{2}}^{2-\frac{1}{r_{2}}}\left(\mathbb{R}^{d-1}\right)$ we need the following commutator estimate.

Lemma 8 Let $a \in B_{r r}^{\tau}\left(\mathbb{R}^{d}\right), \tau>0,1 \leq r \leq \infty$, such that $\tau>\frac{d}{r}$. Then

$$
\left[a(x),\left\langle D_{x}\right\rangle^{s}\right]: H_{q}^{s-\theta}\left(\mathbb{R}^{d}\right) \rightarrow L^{q}\left(\mathbb{R}^{d}\right)
$$

is a bounded operator for all $0 \leq s \leq \tau, 1<q<\infty$ with $q \leq r$ and all $0<\theta<$ $\min \left(1, \tau-\frac{d}{r}\right)$.

The lemma is a consequence of Marschall [43, Corollary 3.4], where we note that $\left[a(x),\left\langle D_{x}\right\rangle^{s}\right]=\left\langle D_{x}\right\rangle^{s} a(x)-O P\left(a(x)\langle\xi\rangle^{s}\right)$.

Next we define a non-smooth variant of the classes of parameter-dependent pseudodifferential operators studied in [37]. To this end, we denote $\rho(\xi, \mu)=\langle\xi\rangle\langle(\xi, \mu)\rangle^{-1}$.

Definition 2 Let $m, v \in \mathbb{R}$. Then $C^{\tau} S_{1,0}^{m, v}\left(\mathbb{R}^{d} \times \overline{\mathbb{R}}_{+}^{d+1}\right)$ is the space of all functions $p(x, \xi, \mu)$ smooth w.r.t. $(\xi, \mu)$ and in $C^{\tau}$ w.r.t. $x$ such that

$$
\left\|D_{\xi}^{\alpha} D_{\mu}^{j} p(., \xi, \mu)\right\|_{C^{\tau}\left(\mathbb{R}^{d}\right)} \leq C_{\alpha, j}\left(\rho(\xi, \mu)^{\nu-|\alpha|}+1\right)\langle\xi, \mu\rangle^{m-|\alpha|-j}
$$

uniformly in $(\xi, \mu) \in \overline{\mathbb{R}}_{+}^{d+1}$ and for all $\alpha \in \mathbb{N}_{0}^{d}, j \in \mathbb{N}_{0}$. Moreover, let

$$
|p|_{k}^{(m, v)}=\sup _{|\alpha|, j \leq k,(\xi, \mu) \in \mathbb{R}_{+}^{d+1}}\left\|D_{\xi}^{\alpha} D_{\mu}^{j} p(., \xi, \mu)\right\|_{C^{\tau}\left(\mathbb{R}^{d}\right)}\left(\rho^{\nu-|\alpha|}+1\right)^{-1}\langle\xi, \mu\rangle^{-m+|\alpha|+j}
$$

be the corresponding increasing sequence of semi-norms.

We note that

$$
\left(\rho(\xi, \mu)^{v}+1\right)\langle\xi, \mu\rangle^{m} \simeq \begin{cases}\langle\xi, \mu\rangle^{m} & \text { if } v \geq 0 \\ \langle\xi\rangle^{v}\langle\xi, \mu\rangle^{m-v} & \text { if } v<0\end{cases}
$$

Remark 4 If $p \in C^{\tau} S_{1,0}^{m, v}$ and $m^{\prime}>m$, then $p \in C^{\tau} S_{1,0}^{m^{\prime}, v}$ with $|p|_{k}^{\left(m^{\prime}, v\right)} \leq\langle\mu\rangle^{m-m^{\prime}}$ $|p|_{k}^{(m, v)}$ for all $k \in \mathbb{N}_{0}$. Moreover, if $m \leq 0, v \geq 0$ and if we look at $p$ as a parameter-independent symbol with fixed $\mu \geq 0$, then $|p(., \mu)|_{k}^{(m)} \leq C|p|_{k}^{(m, v)}$ uniformly in $\mu \in \overline{\mathbb{R}}_{+}$.

In order to deal with the symbols after coordinate transformation, we use the following simple lemma. 
Lemma 9 Let $p(\xi, \mu) \in S_{1,0}^{m, v}\left(\mathbb{R}^{d} \times \overline{\mathbb{R}}_{+}^{d+1}\right), m, v \in \mathbb{R}$, and $A \in C^{\tau}\left(\mathbb{R}^{d}\right)^{d \times d}, \tau>0$, with $A^{-1} \in C^{\tau}\left(\mathbb{R}^{d}\right)^{d \times d}$. Then $q(x, \xi, \mu):=p(A(x) \xi, \mu) \in C^{\tau} S_{1,0}^{m, v}\left(\mathbb{R}^{d} \times \overline{\mathbb{R}}_{+}^{d+1}\right)$, and for every $k \in \mathbb{N}_{0}$ there is some $k^{\prime} \in \mathbb{N}_{0}$ such that $|q|_{k}^{(m, v)} \leq C|p|_{k^{\prime}}^{(m, v)}$, where $C$ depends only on $\|A\|_{C^{\tau}},\left\|A^{-1}\right\|_{C^{\tau}}, k, m, v$, and $d$.

Proof The proof is a simple variant of the proof of [11, Lemma 5.4].

\subsection{Pseudodifferential boundary value problems with non-smooth coefficients}

We recall a non-smooth version of parameter-dependent Green operators developed in [37] as defined in [11] with the only difference that $C^{0,1}$-regularity w.r.t. $x$ is replaced by $C^{\tau}$-regularity for some $\tau>0$. We use the notation of [37] except that $\gamma_{j} u=\left.(-1)^{d} \partial_{d}^{j} u\right|_{\partial \mathbb{R}_{+}^{d}}$. Recall that $\overline{\mathbb{R}}_{++}^{2}=\overline{\mathbb{R}}_{+} \times \overline{\mathbb{R}}_{+}$.

We start with the definition of the symbol-kernels of non-smooth Poisson, trace, and singular Green operators.

Definition 3 The space $C^{\tau} S_{1,0}^{m, v}\left(\mathbb{R}^{N} \times \overline{\mathbb{R}}_{+}^{d}, \mathcal{S}\left(\overline{\mathbb{R}}_{+}\right)\right), m, v \in \mathbb{R}, d, N \in \mathbb{N}$, consists of all functions $\tilde{f}\left(x, \xi^{\prime}, \mu, y_{d}\right)$, which are smooth in $\left(\xi^{\prime}, \mu, y_{d}\right) \in \overline{\mathbb{R}}_{+}^{d} \times \overline{\mathbb{R}}_{+}$, are in $C^{\tau}\left(\mathbb{R}^{N}\right)$ with respect to $x$, and satisfy

$$
\begin{aligned}
& \left\|y_{d}^{l} \partial_{y_{d}}^{l^{\prime}} \partial_{\mu}^{j} D_{\xi^{\prime}}^{\alpha} \tilde{f}\left(., \xi^{\prime}, \mu, .\right)\right\|_{C^{\tau}\left(\mathbb{R}^{N} ; L_{y_{d}}^{2}\left(\mathbb{R}_{+}\right)\right)} \\
& \quad \leq C_{\alpha, j, l, l^{\prime}}\left(\rho\left(\xi^{\prime}, \mu\right)^{\nu-\left[l-l^{\prime}\right]_{+}-|\alpha|}+1\right)\left\langle\xi^{\prime}, \mu\right\rangle^{m+\frac{1}{2}-l+l^{\prime}-|\alpha|-j}
\end{aligned}
$$

for all $\alpha \in \mathbb{N}_{0}^{d-1}, j, l, l^{\prime} \in \mathbb{N}_{0}$.

Similarly, the space $C^{\tau} S_{1,0}^{m, v}\left(\mathbb{R}^{N} \times \overline{\mathbb{R}}_{+}^{d}, \mathcal{S}\left(\overline{\mathbb{R}}_{++}^{2}\right)\right), m, v \in \mathbb{R}, d, N \in \mathbb{N}$, is the space of all $\tilde{f}\left(x, \xi^{\prime}, y_{d}, z_{d}\right)$, which are smooth in $\left(\xi^{\prime}, \mu, y_{d}, z_{d}\right) \in \overline{\mathbb{R}}_{+}^{d} \times \overline{\mathbb{R}}_{++}^{2}$ and which are in $C^{\tau}\left(\mathbb{R}^{N}\right)$ with respect to $x$ such that

$$
\begin{aligned}
& \left\|y_{d}^{k} \partial_{y_{d}}^{k^{\prime}} z_{d}^{l} \partial_{z_{d}}^{l^{\prime}} \partial_{\mu}^{j} D_{\xi^{\prime}}^{\alpha} \tilde{f}\left(., \xi^{\prime}, .\right)\right\|_{C^{\tau}\left(\mathbb{R}^{N} ; L_{y_{d}, z_{d}}^{2}\left(\mathbb{R}_{++}^{2}\right)\right)} \\
& \quad \leq C_{\alpha, j, k, k^{\prime}, l, l^{\prime}}\left(\rho^{\nu-\left[k-k^{\prime}\right]_{+}-\left[l-l^{\prime}\right]_{+}-|\alpha|}+1\right)\left\langle\xi^{\prime}, \mu\right\rangle^{m+1-k+k^{\prime}-l+l^{\prime}-|\alpha|-j}
\end{aligned}
$$

for all $\alpha \in \mathbb{N}_{0}^{d-1}, j, k, k^{\prime}, l, l^{\prime} \in \mathbb{N}_{0}$, where $\rho=\rho\left(\xi^{\prime}, \mu\right)$. Finally, $m$ is called the degree of the symbols $f \in C^{\tau} S_{1,0}^{m, v}\left(\mathbb{R}^{N} \times \overline{\mathbb{R}}_{+}^{d}, \mathcal{K}\right), \mathcal{K}=\mathcal{S}\left(\overline{\mathbb{R}}_{+}\right), \mathcal{S}\left(\overline{\mathbb{R}}_{++}^{2}\right)$ ).

Now the Poisson operators with non-smooth coefficients are defined in almost the same way as in the smooth case:

Definition 4 Let $\tilde{k}=\tilde{k}\left(x, \xi^{\prime}, y_{n}\right) \in C^{\tau} S_{1,0}^{m-1, v}\left(\mathbb{R}^{d} \times \overline{\mathbb{R}}_{+}^{d}, \mathcal{S}\left(\overline{\mathbb{R}}_{+}\right)\right), m, v \in \mathbb{R}$. Then we define the Poisson operator of order $m$ by

$$
k\left(x, \mu, D_{x}\right) a=\mathcal{F}_{\xi^{\prime} \mapsto x^{\prime}}^{-1}\left[\tilde{k}\left(x, \xi^{\prime}, \mu, x_{d}\right) a^{\prime}\left(\xi^{\prime}\right)\right], \quad a \in \mathcal{S}\left(\mathbb{R}^{d-1}\right) .
$$


Finally, we note that the boundary symbol operator $k\left(x, \xi^{\prime}, \mu, D_{d}\right): \mathbb{C} \rightarrow \mathcal{S}\left(\overline{\mathbb{R}}_{+}\right)$is defined as a one-dimensional Poisson operator with symbol-kernel $\tilde{k}\left(x, \xi^{\prime}, \mu, y_{d}\right)$ for fixed $\left(x^{\prime}, \xi^{\prime}, \mu\right)$, which is simply defined by

$$
k\left(x, \xi^{\prime}, \mu, D_{d}\right) a=\tilde{k}\left(x, \xi^{\prime}, \mu, x_{d}\right) a \text { for all } a \in \mathbb{C} .
$$

As usually, Poisson operators can be considered as operator-valued pseudodifferential operators with values in $\mathcal{L}(\mathbb{C} ; H)$, where $H$ is a suitable space of functions on $\mathbb{R}_{+}$, e.g., $H^{m}\left(\mathbb{R}_{+}\right)$or $L^{2}\left(\mathbb{R}_{+}, x_{d}^{s}\right), m, s \geq 0$. Having this in mind, $k\left(D_{x}, x^{\prime}, \mu\right)=$ $O P^{\prime}\left(k\left(y^{\prime}, \xi^{\prime}, \mu, D_{d}\right)\right)$ denotes the corresponding pseudodifferential operator in $y$-form as defined in (5.11).

The trace and singular Green operators are defined as follows:

Definition 5 Let $m, v \in \mathbb{R}$ and let $r \in \mathbb{N}_{0}$.

1. If $\tilde{t}_{0} \in C^{\tau} S_{1,0}^{m, v}\left(\mathbb{R}^{d-1} \times \overline{\mathbb{R}}_{+}^{d}, \mathcal{S}\left(\overline{\mathbb{R}}_{+}\right)\right), s_{j} \in C^{\tau} S_{1,0}^{m-j, v}\left(\mathbb{R}^{d-1} \times \mathbb{R}^{d-1}\right), j=$ $0, \ldots, r-1$, then the associated trace operator of order $m$ and class $r$ is defined as

$$
\begin{aligned}
t\left(x^{\prime}, \mu, D_{x}\right) f & =\sum_{j=0}^{r-1} s_{j}\left(x^{\prime}, \mu, D_{x^{\prime}}\right) \gamma_{j} f+t_{0}\left(x^{\prime}, \mu, D_{x}\right) f \\
t_{0}\left(x^{\prime}, \mu, D_{x}\right) f & =\mathcal{F}_{\xi^{\prime} \mapsto x^{\prime}}^{-1}\left[\int_{0}^{\infty} \tilde{t}_{0}\left(x^{\prime}, \xi^{\prime}, \mu, y_{d}\right) f^{\prime}\left(\xi^{\prime}, y_{d}\right) d y_{d}\right],
\end{aligned}
$$

where $f^{\prime}\left(\xi^{\prime}, x_{d}\right)=\mathcal{F}_{x^{\prime} \mapsto \xi^{\prime}}\left[f\left(., x_{d}\right)\right]$.

2. If $\tilde{g}_{0} \in C^{\tau} S_{1,0}^{m-1, v}\left(\mathbb{R}^{d} \times \overline{\mathbb{R}}_{+}^{d}, \mathcal{S}\left(\overline{\mathbb{R}}_{++}^{2}\right)\right), \tilde{k}_{j} \in C^{\tau} S_{1,0}^{m-j-1, v}\left(\mathbb{R}^{d} \times \overline{\mathbb{R}}_{+}^{d}, \mathcal{S}\left(\overline{\mathbb{R}}_{+}\right)\right)$for $j=0, \ldots, r-1$, then the associated singular Green operator of order $m$ and class $r$ is defined as

$$
\begin{aligned}
g\left(x, \mu, D_{x}\right) f & =\sum_{j=0}^{r-1} k_{j}\left(x, \mu, D_{x}\right) \gamma_{j} f+g_{0}\left(x, \mu, D_{x}\right) f, \\
g_{0}\left(x, \mu, D_{x}\right) f & =\mathcal{F}_{\xi^{\prime} \mapsto x^{\prime}}^{-1}\left[\int_{0}^{\infty} \tilde{g}_{0}\left(x, \xi^{\prime}, \mu, x_{d}, y_{d}\right) f^{\prime}\left(\xi^{\prime}, y_{d}\right) d y_{d}\right],
\end{aligned}
$$

where $f$ is as above and $k_{j}\left(x, \mu, D_{x}\right)$ denotes the Poisson operator with symbolkernel $\tilde{k}_{j}\left(x, \xi^{\prime}, \mu, y_{d}\right)$ (in $x$-form).

Finally, the boundary symbol operators $t\left(x^{\prime}, \xi^{\prime}, \mu, D_{d}\right), g\left(x, \xi^{\prime}, \mu, D_{d}\right)$ and the corresponding operators in $R$-form $t\left(D_{x}, x^{\prime}\right), g\left(D_{x}, x\right)$ are defined in the same way as for the Poisson operator. Note that, if $t^{\prime}\left(x^{\prime}, \mu, D_{x}\right)$ is a trace operator of class 0 , then

$$
\left(t\left(x^{\prime}, \mu, D_{x}\right) \varphi, \psi\right)_{\mathbb{R}^{d-1}}=\left(\varphi, k\left(D_{x}, x^{\prime}, \mu\right) \psi\right)_{\mathbb{R}_{+}^{d}}
$$


where $\tilde{k}\left(x^{\prime}, \xi^{\prime}, \mu, y_{d}\right)=\overline{\tilde{t}\left(x^{\prime}, \xi^{\prime}, \mu, y_{d}\right)}$ and $\varphi \in \mathcal{S}\left(\overline{\mathbb{R}}_{+}^{d}\right), \psi \in \mathcal{S}\left(\mathbb{R}^{d-1}\right)$. Hence trace operators can be considered as adjoints of Poisson operators plus a sum of usual trace operators $s_{j}\left(x^{\prime}, \mu, D_{x^{\prime}}\right) \gamma_{j}$, cf. e.g., [37, Proposition 2.4.2]. Moreover, if $k\left(x, \mu, D_{x}\right)$ is a Poisson operator, then

$$
\left(k\left(x, \mu, D_{x}\right) \psi, \varphi\right)_{\mathbb{R}_{+}^{d}}=\left(\psi, t\left(D_{x}, x, \mu\right) \varphi\right)_{\mathbb{R}^{d-1}},
$$

where $\tilde{t}\left(x, \xi^{\prime}, \mu, y_{d}\right)=\overline{\tilde{k}\left(x, \xi^{\prime}, y_{d}\right)}$ and $\varphi \in \mathcal{S}\left(\overline{\mathbb{R}}_{+}^{d}\right), \psi \in \mathcal{S}\left(\mathbb{R}^{d-1}\right)$. Similarly, if $g_{0}\left(x^{\prime}, \mu, D_{x}\right)$ is a singular Green operator of class 0 in $x$-form, then

$$
\left(g_{0}\left(x^{\prime}, \mu, D_{x}\right) \varphi, \psi\right)_{\mathbb{R}_{+}^{d}}=\left(\varphi, g_{1}\left(D_{x}, x^{\prime}, \mu\right) \psi\right)_{\mathbb{R}_{+}^{d}}
$$

for all $\varphi, \psi \in \mathcal{S}\left(\overline{\mathbb{R}}_{+}^{d}\right)$, where $\tilde{g}_{1}\left(x, \xi^{\prime}, \mu, y_{d}, z_{d}\right)=\overline{\tilde{g}_{0}\left(x, \xi^{\prime}, \mu, z_{d}, y_{d}\right)}$. We note that most of the time the symbol kernels $\tilde{k}\left(x, \xi^{\prime}, y_{d}\right), \tilde{t}_{0}\left(x, \xi^{\prime}, y_{d}\right)$, and $\tilde{g}_{0}\left(x, \xi^{\prime}, y_{d}, z_{d}\right)$ will be independent of $x_{d}$, which is denoted by $x^{\prime}$ instead of $x$ in the symbol-kernel.

We refer to [37] and [9, Definition 5.2] for the definition of the (global) transmission condition for a pseudodifferential symbol $p \in S_{1,0}^{m}\left(\mathbb{R}^{d} \times \mathbb{R}^{d}\right)$ and a variant for $p \in C^{\tau} S_{1,0}^{m}\left(\mathbb{R}^{d} \times \mathbb{R}^{d}\right)$. We will not use this property directly since we will mainly deal with differential operators or with the mapping property $p\left(D_{x}, x\right)_{+}: L^{q}(\Omega) \rightarrow$ $W_{q}^{2}(\Omega)$ for $p \in C^{\tau} S_{1,0}^{-2}\left(\mathbb{R}^{d} \times \mathbb{R}^{d}\right)$, which holds without the transmission condition. For completeness we recall the general definition of a Green operator with non-smooth coefficients as in [9].

Definition 6 A Green operator (in $L$-form) of order $m \in \mathbb{Z}$, class $r \in \mathbb{N}_{0}$, and regularity $v \in \mathbb{R}$ with coefficients in $C^{\tau}$ is defined as

$$
a\left(x, \mu, D_{x}\right)=\left(\begin{array}{cc}
p\left(x, \mu, D_{x}\right)_{+}+g\left(x^{\prime}, \mu, D_{x}\right) & k\left(x^{\prime}, \mu, D_{x}\right) \\
t\left(x^{\prime}, \mu, D_{x}\right) & s\left(x^{\prime}, \mu, D_{x^{\prime}}\right)
\end{array}\right),
$$

where $k\left(x^{\prime}, \mu, D_{x}\right), t\left(x^{\prime}, \mu, D_{x}\right)$, and $g\left(x^{\prime}, \mu, D_{x}\right)$ are Poisson, trace, and singular Green operators of order $m$, regularity $v$, and class $r$,

$$
p\left(x^{\prime}, \mu, D_{x}\right)_{+}=r^{+} p\left(x^{\prime}, \mu, D_{x}\right) e^{+}, \quad p \in C^{\tau} S_{1,0}^{m, v}\left(\mathbb{R}^{d} \times \overline{\mathbb{R}}_{+}^{d}\right),
$$

is a truncated pseudodifferential operator satisfying the transmission condition in the sense of [9, Definition 5.2] and $s \in C^{\tau} S_{1,0}^{m-1, v}\left(\mathbb{R}^{d-1} \times \overline{\mathbb{R}}_{+}^{d}\right)$.

In the following we will often restrict ourselves to parameter-independent symbols and operators. The corresponding symbol classes $C^{\tau} S_{1,0}^{d}\left(\mathbb{R}^{d-1} \times \mathbb{R}^{d-1}, \mathcal{K}\right), \mathcal{K}=$ $\mathcal{S}\left(\overline{\mathbb{R}}_{+}\right), \mathcal{S}\left(\overline{\mathbb{R}}_{++}^{2}\right)$, are defined as above with the restriction that the symbols are independent of $\mu$ and the symbol estimates hold for $\mu=0$, cf. [9] for details.

Moreover, if $\tilde{f}$ is a Poisson, trace, or singular Green symbol-kernel, then $|\tilde{f}|_{k}^{(m, v)}$, $k \in \mathbb{N}$, are the semi-norms (monotonically increasing in $k$ ) associated to (5.12), (5.13), 
resp., in the usual way, cf. Definitions 1 and 2. The semi-norms of parameter-independent symbols will be denoted by $|\tilde{f}|_{k}^{(m)}$.

Remark 5 1. As in Remark $4,|\tilde{f}|_{k}^{(m+\varepsilon, v)} \leq\langle\mu\rangle^{-\varepsilon}|\tilde{f}|_{k}^{(m, v)}$ for all $\varepsilon>0$.

2. If $\tilde{f}$ is a parameter-dependent Poisson or trace symbol-kernel of degree $m \leq-\frac{1}{2}$, regularity $v \geq 0$, then $\tilde{f}(., \mu), \mu \geq 0$ fixed, is a parameter-independent symbolkernel of the same degree with $|\tilde{f}(., \mu)|_{k}^{(m)} \leq|\tilde{f}|_{k}^{(m, v)}$ uniformly in $\mu>0$. The same is true for parameter-dependent singular Green symbol-kernels of degree $m \leq-1$.

Remark 6 Let $a_{j}\left(x, \xi^{\prime}, D_{d}\right), j=1,2$, be the boundary symbol operator of a Poisson, trace, singular Green operator, or a pseudodifferential operators with the transmission condition of order $m_{j}$ (and class $r_{j}$ ) with coefficients in $C^{\tau_{j}}$. As observed in [9, Remark 4.5], the composition $a_{1}\left(x, \xi^{\prime}, D_{d}\right) a_{2}\left(x^{\prime}, \xi^{\prime}, D_{d}\right)=a\left(x, \xi^{\prime}, D_{d}\right)$ of the boundary symbol operators is again a boundary symbol operator if the composition is well-defined and the coefficients of $a_{2}$ are independent of $x_{d}$. The boundary symbol operator of the composition is also denoted by $\left(a_{1} \circ_{d} a_{2}\right)\left(x, \xi^{\prime}, D_{d}\right)$.

The following theorem summarizes some mapping properties of trace and singular Green operators in $R$-form, which will be used in the following.

Theorem 6 Let $1<q<\infty$.

1. Let $t \in C^{\tau} S_{1,0}^{m}\left(\mathbb{R}^{d-1} \times \mathbb{R}^{d-1}, \mathcal{S}\left(\overline{\mathbb{R}}_{+}\right)\right), m \in \mathbb{R}$, be a trace operator of order $d$ and class 0 . Then $t\left(D_{x}, x^{\prime}\right)$ extend to a bounded operator

$$
t\left(D_{x}, x^{\prime}\right): L^{q}\left(\mathbb{R}_{+}^{d}\right) \rightarrow B_{q q}^{-m-\frac{1}{q}}\left(\mathbb{R}^{d-1}\right) .
$$

2. Let $g \in C^{\tau} S_{1,0}^{-m-1}\left(\mathbb{R}^{d-1} \times \mathbb{R}^{d}, \mathcal{S}\left(\overline{\mathbb{R}}_{++}^{2}\right)\right), m \in \mathbb{R}$, be a singular Green operator of order $-m$ and class 0 . Then $g\left(D_{x}, x^{\prime}\right)$ extends to a bounded operator

$$
g\left(D_{x}, x^{\prime}\right): L^{q}\left(\mathbb{R}_{+}^{d}\right) \rightarrow W_{q}^{m}\left(\mathbb{R}_{+}^{d}\right) .
$$

All operators depend continuously on the symbols with respect to the operator norm and the symbol semi-norms.

Proof The theorem follows directly from [9, Theorem 4.8] and duality using (5.15)(5.16).

The following lemma summarizes the results concerning composition of non-smooth pseudodifferential operators which we need in Sect. 5.6.

Lemma 10 Let $1<q<\infty$ and $d<r \leq \infty$ such that $q \leq r$ and let $d_{1} \in \mathbb{N}_{0}$. Moreover, let $p_{1}\left(x, D_{x}\right)=\sum_{|\alpha| \leq d_{1}} a_{\alpha}(x) D_{x}^{\alpha}$ be a differential operator of order $d_{1}$ with coefficients $a_{\alpha} \in W_{r}^{1}\left(\mathbb{R}^{d}\right), r>d$, for all $|\alpha| \leq d_{1}$ and let $t\left(x^{\prime}, D_{x}\right)=$ $\sum_{|\alpha| \leq d_{1}-1} b_{\alpha}\left(x^{\prime}\right) \gamma_{0} D_{x}^{\alpha}$ be a differential trace operator of order $d_{1}-1$, class $d_{1}$, and with coefficients $b_{\alpha} \in W_{r}^{1-\frac{1}{r}}\left(\mathbb{R}^{d-1}\right)$. 
1. Let $\tilde{g} \in C^{\tau} S_{1,0}^{-d_{1}-1}\left(\mathbb{R}^{d-1} \times \mathbb{R}^{d-1}, \mathcal{S}\left(\overline{\mathbb{R}}_{++}^{2}\right)\right)$. Then

$$
\begin{aligned}
& p_{1}\left(x, D_{x}\right) g\left(D_{x}, x^{\prime}\right)-\left(\left.p_{1}\right|_{x_{d}=0} \circ_{d} g\right)\left(D_{x}, x^{\prime}\right): L^{q}\left(\mathbb{R}_{+}^{d}\right) \rightarrow L^{q}\left(\mathbb{R}_{+}^{d}\right), \\
& t\left(x^{\prime}, D_{x}\right) g\left(D_{x}, x^{\prime}\right)-\left(t \circ_{d} g\right)\left(D_{x}, x^{\prime}\right): L^{q}\left(\mathbb{R}_{+}^{d}\right) \rightarrow B_{q q}^{1-\frac{1}{q}}\left(\mathbb{R}^{d-1}\right), \\
& \gamma_{0} g\left(D_{x}, x^{\prime}\right)-\left(\gamma_{0} \circ_{d} g\right)\left(D_{x}, x^{\prime}\right): L^{q}\left(\mathbb{R}_{+}^{d}\right) \rightarrow B_{q q}^{d_{1}-\frac{1}{q}}\left(\mathbb{R}^{d-1}\right)
\end{aligned}
$$

with operator norms bounded by $C\left(p_{1}\right)|g|_{k}^{\left(-d_{1}-1+\varepsilon\right)}, C(t)|g|_{k}^{\left(-d_{1}-1+\varepsilon\right)}$, resp., for some $\varepsilon, C>0, k \in \mathbb{N}$. Moreover,

$$
\begin{aligned}
& t\left(x^{\prime}, D_{x}\right) g\left(D_{x}, x^{\prime}\right)-\left(t \circ_{d} g\right)\left(D_{x}, x^{\prime}\right): L^{q}\left(\mathbb{R}_{+}^{d}\right) \rightarrow L^{q}\left(\mathbb{R}^{d-1}\right), \\
& \gamma_{0} g\left(D_{x}, x^{\prime}\right)-\left(\gamma_{0} \circ_{d} g\right)\left(D_{x}, x^{\prime}\right): L^{q}\left(\mathbb{R}_{+}^{d}\right) \rightarrow L^{q}\left(\mathbb{R}^{d-1}\right)
\end{aligned}
$$

with operator norm bounded by $C(t)|g|_{k}^{\left(-d_{1}-\frac{1}{q}+\varepsilon\right)}, C|g|_{k}^{\left(-1-\frac{1}{q}+\varepsilon\right)}$, resp., for some $\varepsilon, C>0, k \in \mathbb{N}$.

2. Let $p_{2} \in C^{\tau} S_{1,0}^{-d_{1}}\left(\mathbb{R}^{d} \times \mathbb{R}^{d}\right)$. Then

$$
p_{1}\left(x^{\prime}, D_{x}\right) p_{2}\left(D_{x}, x\right)_{+}-\left(p_{1} \cdot p_{2}\right)\left(D_{x}, x\right)_{+}: L^{q}\left(\mathbb{R}_{+}^{d}\right) \rightarrow L^{q}\left(\mathbb{R}_{+}^{d}\right)
$$

with operator-norms bounded by $C\left(p_{1}\right)\left|p_{2}\right|_{k}^{\left(-d_{1}+\varepsilon\right)}$ for some $\varepsilon, C>0, k \in \mathbb{N}_{0}$. Moreover, if $p_{2}$ satisfies the (global) transmission condition, cf. [9, Definition 5.2], and $d_{1} \geq 1$, then

$$
\begin{aligned}
& t\left(x^{\prime}, D_{x}\right) p_{2}\left(D_{x}, x\right)_{+}-\left(\left.t \circ_{d} p_{2}\right|_{x_{d}=0}\right)\left(D_{x}, x^{\prime}\right): L^{q}\left(\mathbb{R}_{+}^{d}\right) \rightarrow B_{q q}^{1-\frac{1}{q}}\left(\mathbb{R}^{d-1}\right) \\
& \gamma_{0} p_{2}\left(D_{x}, x\right)_{+}-\left(\left.\gamma_{0} \circ_{d} p_{2}\right|_{x_{d}=0}\right)\left(D_{x}, x^{\prime}\right): L^{q}\left(\mathbb{R}_{+}^{d}\right) \rightarrow B_{q q}^{d_{1}-\frac{1}{q}}\left(\mathbb{R}^{d-1}\right)
\end{aligned}
$$

with operator norms bounded by $C(t)\left|p_{2}\right|_{k}^{\left(-d_{1}+\varepsilon\right)}$ for some $\varepsilon, C(t)>0, k \in \mathbb{N}$. Finally,

$$
\begin{aligned}
& t\left(x^{\prime}, D_{x}\right) p_{2}\left(D_{x}, x\right)_{+}-\left(\left.t \circ_{d} p_{2}\right|_{x_{d}=0}\right)\left(D_{x}, x^{\prime}\right): L^{q}\left(\mathbb{R}_{+}^{d}\right) \rightarrow L^{q}\left(\mathbb{R}^{d-1}\right) \\
& \gamma_{0} p_{2}\left(D_{x}, x\right)_{+}-\left(\left.\gamma_{0} \circ_{d} p_{2}\right|_{x_{d}=0}\right)\left(D_{x}, x^{\prime}\right): L^{q}\left(\mathbb{R}_{+}^{d}\right) \rightarrow L^{q}\left(\mathbb{R}^{d-1}\right)
\end{aligned}
$$

with operator norms bounded by $C(t)\left|p_{2}\right|_{k}^{\left(-d_{1}+\frac{1}{q^{\prime}}+\varepsilon\right)}, C\left|p_{2}\right|_{k}^{\left(-\frac{1}{q}+\varepsilon\right)}$, resp.

Proof First we consider the compositions with $p_{1}\left(x, D_{x}\right)$. Since $p_{1}\left(x, D_{x}\right)=\sum_{|\alpha| \leq d_{1}}$ $a_{\alpha}(x) D_{x}^{\alpha}$ and $D_{x}^{\alpha} p_{2}\left(D_{x}, x\right)=O P\left(\xi^{\alpha} p_{2}(y, \xi)\right)$ as well as

$$
D_{x}^{\alpha} g\left(D_{x}, x^{\prime}\right)=\mathrm{OP}^{\prime}\left(\mathrm{OP}_{d}\left(\xi^{\alpha}\right) \circ g\left(y^{\prime}, \xi^{\prime}, D_{d}\right)\right)
$$


it suffices to consider the case $d_{1}=0$ and $p_{1}\left(x, D_{x}\right)=a(x)$. But, using the relations

$$
\begin{aligned}
& \left(a(x) g\left(D_{x}, x\right) \varphi, \psi\right)_{\mathbb{R}_{+}^{d}}=\left(\varphi, g_{1}\left(x, D_{x}\right) \bar{a}(x) \psi\right)_{\mathbb{R}_{+}^{d}}, \\
& \left(a(x) p_{2}\left(D_{x}, x\right)_{+} \varphi, \psi\right)_{\mathbb{R}_{+}^{d}}=\left(\varphi, \overline{p_{2}}\left(x, D_{x}\right)_{+} \bar{a}(x) \psi\right)_{\mathbb{R}_{+}^{d}}
\end{aligned}
$$

for all $\varphi, \psi \in \mathcal{S}\left(\overline{\mathbb{R}}_{+}^{d}\right)$, cf. (5.16), where $\tilde{g}_{1}\left(x^{\prime}, \xi^{\prime}, x_{d}, y_{d}\right)=\overline{\tilde{g}\left(x^{\prime}, \xi^{\prime}, y_{d}, x_{d}\right)}$ and $\overline{p_{2}}(x, \xi)=\overline{p_{2}(x, \xi)}$, the corresponding statements 1.-2. follow from [9, Theorem 3.6, Theorem 5.9] with the choice $0<\theta<\min \left(1,1-\frac{d}{r}\right)$, where $\bar{g}, \overline{p_{2}}$ are considers as symbols of order $-d_{1}+\varepsilon$ for $0<\varepsilon \leq \theta$.

Concerning the compositions with $t\left(x^{\prime}, D_{x}\right)$, one can reduce to the case $t\left(x^{\prime}, D_{x}\right)=$ $a\left(x^{\prime}\right) \gamma_{0}$ and $d_{1}=1$ similarly as before. Therefore

$$
\begin{aligned}
t\left(x^{\prime}, D_{x}\right) g\left(D_{x}, x^{\prime}\right) & =a\left(x^{\prime}\right) \gamma_{0} g\left(D_{x}, x\right), \\
t\left(x^{\prime}, D_{x}\right) p_{2}\left(D_{x}, x^{\prime}\right)_{+} & =a\left(x^{\prime}\right) \gamma_{0} p_{2}\left(D_{x}, x\right)_{+},
\end{aligned}
$$

where $\gamma_{0} g\left(D_{x}, x\right)$ and $\gamma_{0} p_{2}\left(D_{x}, x\right)_{+}$are trace operators of class 0 , cf. Remark 6. Let $\tilde{t}\left(D_{x}, x\right)$ denote one of them and let $s=1-\frac{1}{q}$ if $q \geq 2$ and $s \in\left(1-\frac{1}{q}, 1-\frac{1}{r}\right)$ if $q<2$. Then

$$
\left\langle D_{x^{\prime}}\right\rangle^{s} a\left(x^{\prime}\right) \tilde{t}\left(D_{x}, x^{\prime}\right)=a\left(x^{\prime}\right)\left\langle D_{x^{\prime}}\right\rangle^{s} \tilde{t}\left(D_{x}, x^{\prime}\right)+\left[\left\langle D_{x^{\prime}}\right\rangle^{s}, a\left(x^{\prime}\right)\right] \tilde{t}\left(D_{x}, x^{\prime}\right)
$$

where $\left\langle D_{x}\right\rangle^{s} \tilde{t}\left(D_{x}, x^{\prime}\right)$ is a trace operator of order $-\frac{1}{q}$ if $q \geq 2$ and order $s-1$ if $q<2$. Hence we can apply [9, Theorem 4.13] to the first term [again using (5.14)] and Lemma 8 together with Theorem 6 to the second term to prove the statements of the lemma with $B_{q q}^{1-\frac{1}{q}}\left(\mathbb{R}^{d-1}\right)$ replaced by $H_{q}^{s}\left(\mathbb{R}^{d-1}\right)+B_{q q}^{s}\left(\mathbb{R}^{d-1}\right)$. If $q \geq 2$, then $H_{q}^{s}\left(\mathbb{R}^{d-1}\right)=H_{q}^{1-\frac{1}{q}}\left(\mathbb{R}^{d-1}\right) \hookrightarrow B_{q q}^{1-\frac{1}{q}}\left(\mathbb{R}^{d-1}\right)$, cf. e.g., [51, Sect. 2.3.3, Remark 4]. If $1<q<2$, then $s>1-\frac{1}{q}$ and we use that $H_{q}^{s}\left(\mathbb{R}^{d-1}\right) \hookrightarrow B_{q q}^{1-\frac{1}{q}}\left(\mathbb{R}^{d-1}\right)$, cf. [51, Sect. 2.3.3, Remark 4] again. This finishes the proof.

Lemma 11 Let $\tilde{t}_{0} \in C^{\tau} S_{1,0}^{m}\left(\mathbb{R}^{d} \times \mathbb{R}^{d-1}, \mathcal{S}\left(\overline{\mathbb{R}}_{+}\right)\right)$for some $\tau>0, m \in \mathbb{R}$. Then

$$
\mathrm{OP}^{\prime}\left(\tilde{t}_{0}\left(y, \xi^{\prime}, D_{d}\right)-\tilde{t}_{0}\left(y^{\prime}, 0, \xi^{\prime}, D_{d}\right)\right): L^{q}\left(\mathbb{R}_{+}^{d}\right) \rightarrow B_{q q}^{-m-\frac{1}{q}}\left(\mathbb{R}^{d-1}\right)
$$

with operator norm bounded by $C\left|t_{0}\right|_{k}^{(m-\varepsilon)}$ for some $\varepsilon>0$.

Proof Using (5.15) the result directly follows from [9, Theorem 4.11].

Finally, we need the following simple lemma when dealing with coordinate transformations.

Lemma 12 Let $\tilde{f}\left(\xi^{\prime}, \mu, x_{d}\right) \in C^{\tau} S_{1,0}^{m, v}\left(\mathbb{R}^{d-1} \times \overline{\mathbb{R}}_{+}^{d}, \mathcal{S}\left(\overline{\mathbb{R}}_{+}\right)\right), m, v \in \mathbb{R}, \tau>0$. Moreover, let $A\left(x^{\prime}\right) \in C^{\tau}\left(\mathbb{R}^{d-1}\right)^{(d-1) \times(d-1)}, \tau>0$, such that $A^{-1}\left(x^{\prime}\right) \in C^{\tau}$ $\left(\mathbb{R}^{d-1}\right)^{(d-1) \times(d-1)}, c \in C^{\tau}\left(\mathbb{R}^{d-1} \times \mathbb{R}^{d-1}\right), c\left(x^{\prime}\right) \geq c_{0}>0$. Then $\tilde{g}\left(x^{\prime}, \xi^{\prime}, \mu, x_{d}\right):=$ 
$\tilde{f}\left(A\left(x^{\prime}\right) \xi^{\prime}, \mu, c\left(x^{\prime}\right) x_{d}\right) \in C^{\tau} S_{1,0}^{m, v}\left(\mathbb{R}^{d-1} \times \overline{\mathbb{R}}_{+}^{d}, \mathcal{S}\left(\overline{\mathbb{R}}_{+}\right)\right)$and for every $k \in \mathbb{N}_{0}$ there is some $k^{\prime} \in \mathbb{N}_{0}$ such that

$$
|\tilde{g}|_{k}^{(m, v)} \leq C\left(\|A\|_{C^{\tau}},\left\|A^{-1}\right\|_{C^{\tau}}\right)|\tilde{f}|_{k^{\prime}}^{(m, v)}
$$

The same statement is true if $\tilde{f} \in C^{\tau} S_{1,0}^{m, v}\left(\mathbb{R}^{d-1} \times \overline{\mathbb{R}}_{+}^{d}, \mathcal{S}\left(\overline{\mathbb{R}}_{++}^{2}\right)\right)$ is independent of $x^{\prime}$ and if we set

$$
\tilde{g}\left(x^{\prime}, \xi^{\prime}, \mu, x_{d}, y_{d}\right):=\tilde{f}\left(A\left(x^{\prime}\right) \xi^{\prime}, \mu, c\left(x^{\prime}\right) x_{d}, c\left(x^{\prime}\right) y_{d}\right) .
$$

Proof The proof of the lemma is the same as the proof of [11, Lemma 5.5] just replacing $C^{0,1}$-norms by $C^{\tau}$-norms.

Finally, if $\tilde{k}, \tilde{t} \in S_{1,0}^{m, v}\left(\mathbb{R}^{d-1} \times \overline{\mathbb{R}}_{+}^{d}, \mathcal{S}\left(\overline{\mathbb{R}}_{+}\right)\right)$and $\tilde{g} \in C^{\tau} S_{1,0}^{m, v}\left(\mathbb{R}^{d-1} \times \overline{\mathbb{R}}_{+}^{d}, \mathcal{S}\left(\overline{\mathbb{R}}_{++}^{2}\right)\right)$, then we define for $c>0$ and $a \in \mathbb{C}, f \in \mathcal{S}\left(\overline{\mathbb{R}}_{+}\right)$

$$
\begin{aligned}
& k\left(x^{\prime}, \xi^{\prime}, \mu, c D_{d}\right) a:=\tilde{k}\left(x^{\prime}, \xi^{\prime}, \mu, c^{-1} x_{d}\right) a \\
& t\left(x^{\prime}, \xi^{\prime}, \mu, c D_{d}\right) f:=c^{-1} \int_{0}^{\infty} \tilde{t}\left(x^{\prime}, \xi^{\prime}, \mu, c^{-1} y_{d}\right) f\left(y_{d}\right) d y_{d}, \\
& g\left(x^{\prime}, \xi^{\prime}, \mu, c D_{d}\right) f:=c^{-1} \int_{0}^{\infty} \tilde{g}\left(x^{\prime}, \xi^{\prime}, \mu, c^{-1} x_{d}, c^{-1} y_{d}\right) f\left(y_{d}\right) d y_{d} .
\end{aligned}
$$

These definitions are motivated by the relations

$$
k\left(., c D_{d}\right)=\delta_{c^{-1}} k\left(., D_{d}\right), t\left(., c D_{d}\right)=t\left(., D_{d}\right) \delta_{c}, g\left(., c D_{d}\right)=\delta_{c^{-1}} g\left(., D_{d}\right) \delta_{c},
$$

where $\delta_{r} f\left(x_{d}\right)=f\left(r x_{d}\right)$ for $r>0$, where we note that

$$
\delta_{c^{-1}} p\left(D_{x_{d}}\right) \delta_{c}=\mathrm{OP}_{d}\left(p\left(c \xi_{d}\right)\right)
$$

for every suitable function $p: \mathbb{R} \rightarrow \mathbb{R}$. Because of the latter relation, the scaling $D_{d} \mapsto$ $c D_{d}$ is consistent with composition of operators in the sense that $a_{1}\left(., c D_{d}\right) a_{2}\left(., c D_{d}\right)$ $=\left(a_{1} \circ_{d} a_{2}\right)\left(., c D_{d}\right)$ for any Poisson, trace, and singular Green operators $a_{j}, j=1,2$, such that the composition is well-defined. Finally, we note that the choice of the scaling above differs slightly from the one used in [11, Sect. 5.2].

5.3 The model operators of the reduced Stokes equations in $\mathbb{R}_{+}^{d}$ with unit viscosity

In this section we summarize some results on the boundary symbol operator of the reduced Stokes equation in $\mathbb{R}_{+}^{d}$ with unit viscosity as discussed in [11, Sect. 5].

In the following we use the relation $\lambda=e^{i \theta} \mu^{2}$ for $\mu>0, \theta \in(-\delta, \delta)$ respectively $\lambda \in \Sigma_{\delta}$ for some $\delta \in(0, \pi)$ arbitrary but fixed. Most of the time we will write all 
symbol-kernels and boundary symbol operators in dependence of $\lambda \in \Sigma_{\delta}$ instead of $\mu$ having in mind that in the estimates for the symbol-kernel classes the latter relation for $\mu$ and $\lambda$ is used.

First of all, let

$$
a_{j, \lambda}^{r}\left(\xi^{\prime}, D_{d}\right)=\left(\begin{array}{r}
\mu^{2} e^{i \theta}+\left|\xi^{\prime}\right|^{2}+D_{d}^{2}+k_{j}^{r}\left(\xi^{\prime}, D_{d}\right) t_{j}^{r}\left(\xi^{\prime}, D_{d}\right) \\
t_{j}^{\prime}\left(\xi^{\prime}, D_{d}\right)
\end{array}\right),
$$

$j=0,1, \theta \in(-\pi, \pi)$, be the model operator of the reduced Stokes equations, where

$$
\begin{aligned}
& k_{0}^{r}\left(\xi^{\prime}, D_{d}\right) a=e^{-\left[\xi^{\prime}\right] x_{d}}\left(\begin{array}{c}
i \xi^{\prime} \\
{\left[\xi^{\prime}\right]} \\
-1
\end{array}\right) a, \quad k_{1}^{r}\left(\xi^{\prime}, D_{d}\right) a=e^{-\left[\xi^{\prime}\right] x_{d}}\left(\begin{array}{c}
i \xi^{\prime} \\
-\left[\xi^{\prime}\right]
\end{array}\right) a, \\
& t_{0}^{r}\left(\xi^{\prime}, D_{d}\right) u=i \xi^{\prime T} \partial_{d} u^{\prime}(0), \quad t_{1}^{r}\left(\xi^{\prime}, D_{d}\right) u=2 \partial_{d} u_{d}(0), \\
& t_{0}^{\prime}\left(\xi^{\prime}, D_{d}\right) u=u(0), \quad t_{1}^{\prime}\left(\xi^{\prime}, D_{d}\right) u=\left(\begin{array}{c}
i \xi^{\prime} u_{d}(0)+\partial_{d} u^{\prime}(0) \\
i \xi^{\prime} \cdot u^{\prime}(0)+\partial_{d} u_{n}(0)
\end{array}\right)
\end{aligned}
$$

for $a \in \mathbb{C}^{d}$ and $u \in \mathcal{S}\left(\overline{\mathbb{R}}_{+}\right)^{d}$.

We note that these model operators are obtained by considering the reduced Stokes system (3.3)-(3.6) with unit viscosity $v(x) \equiv 1$ in $\Omega=\mathbb{R}_{+}^{d}$ and applying Fourier transformation in tangential direction $x^{\prime} \in \mathbb{R}^{d-1}$. In that case either only the Dirichlet boundary condition (3.5) is considered, which corresponds to the case $j=0$ above and the choice $\Gamma_{1}=\partial \mathbb{R}_{+}^{d}$ and $\Gamma_{2}=\emptyset$, or only the Neumann type boundary condition (3.6) is present, which is denoted by $j=1$ above and is obtained by choosing $\Gamma_{1}=\emptyset, \Gamma_{2}=\partial \mathbb{R}_{+}^{d}$. Here $P$ is replaced by $k_{j}^{r}\left(\xi^{\prime}, D_{d}\right) t_{j}\left(\xi^{\prime}, D_{d}\right)$ since (1.8)-(1.9) is in the case $v \equiv 1$ the weak formulation of the Laplace equation $\Delta p_{1}=0$ together with either Neumann $(j=0)$ or Dirichlet boundary condition $(j=1)$. Calculating the solution of (1.8)-(1.9) explicitly in this case $\Omega=\mathbb{R}_{+}^{d}, v \equiv 1$ after (partial) Fourier transformation, one obtains $k_{j}^{r}\left(\xi^{\prime}, D_{d}\right) t_{j}\left(\xi^{\prime}, D_{d}\right) u$ for given $u$.

Note that the definition of $t_{0}^{r}\left(\xi^{\prime}, D_{d}\right)$ and $k_{0}^{r}\left(\xi^{\prime}, D_{d}\right)$ differs from the definitions in [11], but the product $k_{0}^{r}\left(\xi^{\prime}, D_{d}\right) t_{0}^{r}\left(\xi^{\prime}, D_{d}\right)$ stays the same. - The present decomposition is more suitable for the following. Here [.] denotes a smooth function with $\left[\xi^{\prime}\right]=\left|\xi^{\prime}\right|$ if $\left|\xi^{\prime}\right| \geq 1$ and $\left[\xi^{\prime}\right] \geq \frac{1}{2}$ if $\left|\xi^{\prime}\right|<1$.

The following theorem summarizes the essential properties of the model operator shown in [11].

Theorem 7 Let $0<\delta<\pi$ and let $\theta \in[-\delta, \delta]$. Then there is some $c_{0}>0$ such that

$$
a_{j, \lambda}^{r}\left(\xi^{\prime}, \mu, D_{d}\right) \equiv a_{j}^{r}\left(\xi^{\prime}, \mu, D_{d}\right): H_{2}^{2}\left(\mathbb{R}_{+}\right)^{d} \rightarrow L^{2}\left(\mathbb{R}_{+}\right)^{d} \times \mathbb{C}^{d}
$$

is bijective for all $\left|\left(\xi^{\prime}, \mu\right)\right| \geq c_{0}$. Moreover, $a_{j}^{r}\left(\xi^{\prime}, \mu, D_{d}\right)^{-1}$ is a boundary symbol operator of order -2 , class 0 , and regularity $\frac{1}{2}$. Finally,

$$
a_{j}^{r}\left(\xi^{\prime}, \mu, D_{d}\right)^{-1}\left(\begin{array}{l}
f \\
0
\end{array}\right)=p_{\lambda}\left(\xi^{\prime}, D_{d}\right)_{+} f+g_{j, \lambda}^{r}\left(\xi^{\prime}, D_{d}\right) f \equiv r_{j, \lambda}^{r}\left(\xi^{\prime}, D_{d}\right) f
$$


for $f \in \mathcal{S}\left(\overline{\mathbb{R}}_{+}\right)^{d}$, where $\lambda=e^{i \theta} \mu^{2}, p_{\lambda}(\xi)=\left(\lambda+|\xi|^{2}\right)^{-1}$ and $g_{j, \lambda}^{r}\left(\xi^{\prime}, D_{d}\right)$ satisfies

$$
\left\|\int_{\Gamma_{R}} h(-\lambda) D_{\xi^{\prime}}^{\alpha^{\prime}} g_{j, \lambda}^{r}\left(\xi^{\prime}, D_{d}\right) d \lambda\right\|_{X} \leq C_{\delta, \delta^{\prime}, \alpha^{\prime}}\left\langle\xi^{\prime}\right\rangle^{-\left|\alpha^{\prime}\right|}\|h\|_{\infty}
$$

for $X=\mathcal{L}\left(L^{2}\left(\mathbb{R}_{+} ; x_{d}^{-\delta^{\prime}}\right), H_{2}^{\delta^{\prime}}\left(\mathbb{R}_{+}\right)\right)$and $X=\mathcal{L}\left(H_{2}^{-\delta^{\prime}}\left(\mathbb{R}_{+}\right), L^{2}\left(\mathbb{R}_{+} ; x_{d}^{\delta^{\prime}}\right)\right)$ uniformly in $\xi^{\prime} \in \mathbb{R}^{d-1}$ for all $h \in H(\delta), 0 \leq \delta^{\prime}<\frac{1}{2}, \alpha^{\prime} \in \mathbb{N}_{0}^{d-1}$. Here $\Gamma_{R}=\Gamma \backslash B_{R}$ and $\Gamma=\partial \Sigma_{\delta}$ for some $R \geq R_{0}:=c_{0}^{2}$.

Proof The first part is the content of [11, Lemma 5.1]. The validity of (5.17) follows from [11, Theorem 5.13]. More precisely, from [11, Theorem 5.13] we obtain that

$$
\left\|\int_{\Gamma_{R}} h(-\lambda) D_{\xi^{\prime}}^{\alpha^{\prime} \check{g}_{j, \lambda}^{r}}\left(\xi^{\prime}, D_{d}\right) d \lambda\right\|_{X} \leq C_{\delta, \delta^{\prime}, \alpha^{\prime}}\left\langle\xi^{\prime}\right\rangle^{-\left|\alpha^{\prime}\right|}\|h\|_{\infty}
$$

for $X=\mathcal{L}\left(L^{2}\left(\mathbb{R} ;\left|x_{d}\right|^{-\delta^{\prime}}\right), H_{2}^{\delta^{\prime}}(\mathbb{R})\right)$ and $X=\mathcal{L}\left(H_{2}^{-\delta^{\prime}}(\mathbb{R}), L^{2}\left(\mathbb{R} ;\left|x_{d}\right|^{\delta^{\prime}}\right)\right)$ uniformly in $\xi^{\prime} \in \mathbb{R}^{d-1}$ for all $h \in H(\delta), 0 \leq \delta^{\prime}<\frac{1}{2}, \alpha^{\prime} \in \mathbb{N}_{0}^{d-1}$, where

$$
\check{g}_{j, \lambda}^{r}\left(\xi^{\prime}, D_{d}\right) f:=\int_{\mathbb{R}} \tilde{g}_{j, \lambda}^{r}\left(\xi^{\prime}, x_{d}, y_{d}\right) f\left(y_{d}\right) d y_{d}
$$

for $f \in L^{2}\left(\mathbb{R} ;\left|x_{d}\right|^{-\delta^{\prime}}\right) \cup H_{2}^{-\delta^{\prime}}(\mathbb{R})$ and $\tilde{g}_{j, \lambda}^{r}\left(\xi^{\prime}, x_{d}, y_{d}\right)$ is extended by zero for $x_{d}<0$ or $y_{d}<0$. Since $g_{j, \lambda}^{r}\left(\xi^{\prime}, D_{d}\right)=r_{+} \check{g}_{j, \lambda}^{r}\left(\xi^{\prime}, D_{d}\right) e_{+}$and $e_{+}: H_{2}^{-\delta^{\prime}}\left(\mathbb{R}_{+}\right) \rightarrow H_{2}^{-\delta^{\prime}}(\mathbb{R})$ is continues for $0 \leq \delta^{\prime}<\frac{1}{2}$, (5.17) follows.

Furthermore, we note that

$$
\mathrm{OP}_{d}\left(\lambda+|\xi|^{2}\right) g_{j, \lambda}^{r}\left(\xi^{\prime}, D_{d}\right) f=-k_{j}^{r}\left(\xi^{\prime}, D_{d}\right) t_{j}^{r}\left(\xi^{\prime}, D_{d}\right) r_{j, \lambda}^{r}\left(\xi^{\prime}, D_{d}\right)
$$

since $\mathrm{OP}_{d}\left(\lambda+|\xi|^{2}\right) p_{\lambda}\left(\xi^{\prime}, D_{d}\right) f=f$.

5.4 The model operators of the reduced Stokes equations in $\mathbb{R}_{+}^{d}$ with general viscosity

First of all, we note that, if $(v, p)$ is a solution of the Stokes equation resolvent equation in $\mathbb{R}_{+}^{d}$ for $v \equiv$ const. $>0$, then $(w, q)$ with $w(x)=v\left(v^{\frac{1}{2}} x\right)$ and $q(x)=v^{-\frac{1}{2}} p\left(v^{\frac{1}{2}} x\right)$ is a solution of the Stokes equation with unit viscosity. This scaling is also valid on the level of the boundary symbol operators for the reduced Stokes system as follows: 
After partial Fourier transformation the reduced Stokes equation on $\mathbb{R}_{+}^{d}$ with constant viscosity $v$ becomes

$$
\begin{gathered}
\left(\lambda+v\left|\xi^{\prime}\right|^{2}+v D_{d}^{2}\right) \tilde{u}\left(x_{d}\right)+k_{j}^{r}\left(\xi^{\prime}, D_{d}\right) v t_{j}^{r}\left(\xi^{\prime}, D_{d}\right) \tilde{u}\left(x_{d}\right)=\tilde{f}\left(x_{d}\right), \quad x_{d}>0, \\
v^{j} t_{j}^{\prime}\left(\xi^{\prime}, D_{d}\right) \tilde{u}=\tilde{a}
\end{gathered}
$$

provided that $\left|\xi^{\prime}\right| \geq 1$ where $k_{j}^{r}, t_{j}^{r}, t_{j}^{\prime}$ are as in the previous section. Now we use that

$$
\begin{gathered}
k_{j}^{r}\left(\xi^{\prime}, D_{d}\right) v t_{j}^{r}\left(\xi^{\prime}, D_{d}\right)=k_{j}^{r}\left(v^{\frac{1}{2}} \xi^{\prime}, v^{\frac{1}{2}} D_{d}\right) t_{j}^{r}\left(v^{\frac{1}{2}} \xi^{\prime}, v^{\frac{1}{2}} D_{d}\right) \\
v^{j \frac{1}{2}} t_{j}^{\prime}\left(\xi^{\prime}, D_{d}\right)=t_{j}^{\prime}\left(v^{\frac{1}{2}} \xi^{\prime}, v^{\frac{1}{2}} D_{d}\right)
\end{gathered}
$$

Altogether we see that the boundary symbol operator of the reduced Stokes equation in $\mathbb{R}_{+}^{d}$ with viscosity $v>0$ is

$$
a_{j, \lambda, \nu}^{r}\left(\xi^{\prime}, D_{d}\right):=\left(\begin{array}{cc}
I & 0 \\
0 & v^{\frac{j}{2}}
\end{array}\right) a_{j, \lambda}^{r}\left(v^{\frac{1}{2}} \xi^{\prime}, v^{\frac{1}{2}} D_{d}\right),
$$

where $a_{j, \lambda}^{r}\left(\xi^{\prime}, D_{d}\right) \equiv a_{j}^{r}\left(\xi^{\prime}, \mu, D_{d}\right)$ is the boundary symbol operator of the reduced Stokes equation with unit viscosity as defined above and the factor $v^{\frac{j}{2}}$ only acts on the boundary data.

Finally, we note that there is some $\tilde{g}_{j, \lambda} \in C^{\tau} S_{1,0}^{-2, \frac{1}{2}}\left(\mathbb{R}^{d} \times \overline{\mathbb{R}}^{d+1}\right.$ ) (independent of $x$ ) such that

$$
\begin{aligned}
\left(\begin{array}{c}
i \xi^{\prime} \\
\partial_{d}
\end{array}\right) v^{\frac{1}{2}} g_{j, \lambda}\left(v^{\frac{1}{2}} \xi^{\prime}, v^{\frac{1}{2}} D_{d}\right) & \equiv k_{j}^{r}\left(v^{\frac{1}{2}} \xi^{\prime}, v^{\frac{1}{2}} D_{d}\right) t_{j}^{r}\left(v^{\frac{1}{2}} \xi^{\prime}, v^{\frac{1}{2}} D_{d}\right) r_{j, \lambda}^{r}\left(v^{\frac{1}{2}} \xi^{\prime}, v^{\frac{1}{2}} D_{d}\right) \\
& =-\mathrm{OP}_{d}\left(\lambda+v|\xi|^{2}\right)_{+} g_{j, \lambda}^{r}\left(v^{\frac{1}{2}} \xi^{\prime}, v^{\frac{1}{2}} D_{d}\right)
\end{aligned}
$$

because of (5.18). In particular, this implies

$$
\begin{aligned}
\left.\left(-\partial_{d}\right)^{1-j} v^{\frac{1}{2}} g_{j, \lambda}\left(v^{\frac{1}{2}} \xi^{\prime}, v^{\frac{1}{2}} D_{d}\right)\right|_{x_{d}=0} & =v^{\frac{j}{2}} t_{j}^{r}\left(v^{\frac{1}{2}} \xi^{\prime}, v^{\frac{1}{2}} D_{d}\right) r_{j, \lambda}^{r}\left(v^{\frac{1}{2}} \xi^{\prime}, v^{\frac{1}{2}} D_{d}\right) \\
& =v t_{j}^{r}\left(\xi^{\prime}, D_{d}\right) r_{j, \lambda}^{r}\left(v^{\frac{1}{2}} \xi^{\prime}, v^{\frac{1}{2}} D_{d}\right)
\end{aligned}
$$

and

$$
\left(\left|\xi^{\prime}\right|^{2}-\partial_{d}^{2}\right) g_{j, \lambda}\left(v^{\frac{1}{2}} \xi^{\prime}, v^{\frac{1}{2}} D_{d}\right)=0 \quad \text { in }(0, \infty) .
$$

5.5 Symbols of the reduced Stokes equations in $\mathbb{R}_{\gamma}^{d}$

As we have seen in Sect. 4 coordinate transformation acts on the principal symbol as

$$
a(\xi) \rightsquigarrow \underline{a}\left(x^{\prime}, \xi\right)=a(A(x) \xi)
$$


with an additional factor $U^{T}(x)$ on the left if the range of the operator consists of vector fields and additional factor $U(x)$ on the right if the domain of the operator consists of vector fields. Therefore we define the principal boundary symbol operator for the reduced Stokes equation $\mathbb{R}_{\gamma}^{d}$ by

$$
\begin{aligned}
& \underline{a}_{j, \lambda}^{r}\left(x^{\prime}, \xi^{\prime}, D_{d}\right) \\
& \quad=U^{T}\left(x^{\prime}\right) \operatorname{diag}\left(I, \underline{v}\left(x^{\prime}\right)^{j \frac{1}{2}}\right) a_{j, \lambda}^{r}\left(\underline{v}\left(x^{\prime}\right)^{\frac{1}{2}} A^{\prime}\left(x^{\prime}\right) \xi^{\prime}, \underline{v}\left(x^{\prime}\right)^{\frac{1}{2}} D_{d}\right) U\left(x^{\prime}\right),
\end{aligned}
$$

where $\underline{v}=F^{*} v$ and $\underline{v}\left(x^{\prime}\right)=\underline{v}\left(x^{\prime}, 0\right)$. Hence

$$
\begin{aligned}
& \underline{a}_{j, \lambda}^{r,-1}\left(x^{\prime}, \xi^{\prime}, D_{d}\right) \\
& \quad=U^{T}\left(x^{\prime}\right) a_{j, \lambda}^{r,-1}\left(\underline{\nu}\left(x^{\prime}\right)^{\frac{1}{2}} A^{\prime}\left(x^{\prime}\right) \xi^{\prime}, \underline{v}\left(x^{\prime}\right)^{\frac{1}{2}} D_{d}\right) \operatorname{diag}\left(1, \underline{v}\left(x^{\prime}\right)^{-\frac{j}{2}}\right) U\left(x^{\prime}\right) .
\end{aligned}
$$

This is the essential formula for the construction of the parametrix.

Moreover, we set

$$
\underline{r}_{j, \lambda}^{r}\left(x^{\prime}, 0, \xi^{\prime}, D_{d}\right) f=\underline{a}_{j, \lambda}^{r,-1}\left(x^{\prime}, \xi^{\prime}, D_{d}\right)\left(\begin{array}{l}
f \\
0
\end{array}\right), \quad f \in \mathcal{S}\left(\overline{\mathbb{R}}_{+}\right) .
$$

Then

$$
\underline{r}_{j, \lambda}^{r}\left(x^{\prime}, 0, \xi^{\prime}, D_{d}\right) f=\underline{p}_{\lambda}\left(x^{\prime}, 0, \xi^{\prime}, D_{d}\right)_{+} f+\underline{g}_{j, \lambda}^{r}\left(x^{\prime}, \xi^{\prime}, D_{d}\right) f
$$

where $\underline{p}_{\lambda}(x, \xi)=\left(\lambda+\underline{v}(x)|A(x) \xi|^{2}\right)^{-1}$ and

$$
\underline{g}_{j, \lambda}^{r}\left(x^{\prime}, \xi^{\prime}, D_{d}\right) f=U\left(x^{\prime}\right)^{T} g_{j, \lambda}^{r}\left(\underline{v}^{\frac{1}{2}}\left(x^{\prime}\right) A^{\prime}\left(x^{\prime}\right) \xi^{\prime}, \underline{v}^{\frac{1}{2}}\left(x^{\prime}\right) D_{d}\right) U\left(x^{\prime}\right) f .
$$

Finally, we set for $x=\left(x^{\prime}, x_{d}\right)$ with $x_{d}>0$

$$
\underline{r}_{j, \lambda}^{r}\left(x, \xi^{\prime}, D_{d}\right) f=\underline{p}_{\lambda}\left(x, \xi^{\prime}, D_{d}\right)_{+} f+\underline{g}_{j, \lambda}^{r}\left(x^{\prime}, \xi^{\prime}, D_{d}\right) f
$$

and we define the parametrix of the reduced Stokes system on the transformed $\mathbb{R}_{\gamma}^{d}$ as

$$
\underline{r}_{j, \lambda}^{r}\left(D_{x}, x\right)=\underline{p}_{\lambda}\left(D_{x}, x\right)_{+}+\underline{g}_{j, \lambda}^{r}\left(D_{x}, x^{\prime}\right)
$$

For the general construction of a parametrix in the case of non-smooth coefficients we refer to [9, Sect. 6].

Remark 7 We note that $p_{\lambda}(\xi)=\left(\lambda+|\xi|^{2}\right)^{-1}$ satisfies the transmission condition because of [37, Theorem 2.2.13] and since every polynomial in $\xi$ satisfies the transmission condition. Therefore $\underline{p}_{\lambda}(x, \xi)$ satisfies the global transmission condition in the sense of [9, Definition 5.2] because of [9, Remark 5.3]. 
We have to estimate the semi-norms of the transformed symbols. Because of (4.1) and $\nabla^{\prime} \gamma \in W_{r_{2}}^{1-\frac{1}{r_{2}}}\left(\mathbb{R}^{d-1}\right) \hookrightarrow C^{\tau_{2}}\left(\mathbb{R}^{d-1}\right)$ with $\tau_{2}=1-\frac{d}{r_{2}}>0$, we have $A^{\prime}\left(x^{\prime}\right)$, $A^{\prime-1}\left(x^{\prime}\right), c\left(x^{\prime}\right) \in C^{\tau_{2}}\left(\mathbb{R}^{d-1}\right)$. Moreover, $\left.\underline{v}(x)\right|_{x_{d}=0} \in W_{r_{1}}^{1-\frac{1}{r_{1}}}\left(\mathbb{R}^{d-1}\right) \hookrightarrow C^{\tau_{1}}\left(\mathbb{R}^{d-1}\right)$ with $\tau_{1}=1-\frac{d}{r_{1}}>0$. Hence we can apply Lemmas 9 and 12 to obtain:

Corollary 3 Let $\underline{a}_{j, \lambda}^{r}\left(x^{\prime}, \xi^{\prime}, D_{d}\right), j=0,1$, be the transformed boundary symbol operators of the reduced Stokes equations defined above. Then

$$
\underline{a}_{j}^{r}\left(x^{\prime}, \xi^{\prime}, \mu, D_{d}\right) \equiv \underline{a}_{j, \lambda}^{r}\left(x^{\prime}, \xi^{\prime}, D_{d}\right)
$$

and $\underline{r}_{j}^{r,-1}\left(x, \xi^{\prime}, \mu, D_{d}\right)$ are Green symbols of order $2,-2$, respectively, regularity $\frac{1}{2}$, and $C^{\tau}$-smoothness in $x^{\prime}$ for $\tau=\min \left(1-\frac{d}{r_{1}}, 1-\frac{d}{r_{2}}\right)$. Moreover, the semi-norms of the symbols are uniformly bounded in $\theta \in[-\delta, \delta]$ for any $\delta \in(0, \pi)$.

Theorem 8 Let $\delta \in(0, \pi), R_{0}=c_{0}^{2}>0$ be the constant in Theorem 7 , and $\underline{g}_{j, \lambda}^{r}\left(x^{\prime}, \xi^{\prime}, D_{d}\right)$ be defined as in (5.23) with $j=0,1$. Then

$$
\left\|\int_{\Gamma_{R}} h(-\lambda) \underline{g}_{j, \lambda}^{r}\left(D_{x}, x^{\prime}\right) d \lambda\right\|_{\mathcal{L}\left(L^{q}\left(\mathbb{R}_{+}^{d}\right)\right)} \leq C_{\delta}\|h\|_{\infty}
$$

for every $h \in H(\delta)$ and $R \geq \max \left\{R_{0}, 1\right\}$.

Proof By (5.23) and (5.17), we obtain

$$
\left\|\int_{\Gamma_{R}} h(-\lambda) D_{\xi^{\prime}}^{\alpha^{\prime}} \underline{g}_{j, \lambda}^{r}\left(., \xi^{\prime}, D_{d}\right) d \lambda\right\|_{C^{\tau}(X)} \leq C_{\delta, \delta^{\prime}, \alpha^{\prime}}\left\langle\xi^{\prime}\right\rangle^{-\left|\alpha^{\prime}\right|}\|h\|_{\infty}
$$

for $X=\mathcal{L}\left(L^{2}\left(\mathbb{R}_{+} ; x_{d}^{-\delta^{\prime}}\right), H_{2}^{\delta^{\prime}}\left(\mathbb{R}_{+}\right)\right)$and $X=\mathcal{L}\left(H_{2}^{-\delta^{\prime}}\left(\mathbb{R}_{+}\right), L^{2}\left(\mathbb{R}_{+} ; x_{d}^{\delta^{\prime}}\right)\right)$ uniformly in $\xi^{\prime} \in \mathbb{R}^{d-1}$ for all $h \in H(\delta), 0 \leq \delta^{\prime}<\frac{1}{2}, \alpha^{\prime} \in \mathbb{N}_{0}^{d-1}$. Hence Theorem 5 implies

$$
\left\|\int_{\Gamma_{R}} h(-\lambda) \underline{g}_{j, \lambda}^{r}\left(D_{x}, x^{\prime}\right) d \lambda\right\|_{\mathcal{L}\left(L^{q}\left(\mathbb{R}^{d-1} ; H_{0}\right), L^{q}\left(\mathbb{R}^{d-1} ; H_{1}\right)\right)} \leq C_{\delta}\|h\|_{\infty}
$$

where $\left(H_{0}, H_{1}\right)$ are $\left(L^{2}\left(\mathbb{R}_{+} ; x_{d}^{-\delta^{\prime}}\right), H_{2}^{\delta^{\prime}}\left(\mathbb{R}_{+}\right)\right)$or $\left(H_{2}^{-\delta^{\prime}}\left(\mathbb{R}_{+}\right), L^{2}\left(\mathbb{R}_{+} ; x_{d}^{\delta^{\prime}}\right)\right)$. Now, if $1<q \leq 2$, then one uses the interpolation inclusions

$$
\begin{gathered}
\left(L^{2}\left(\mathbb{R}_{+}, x_{n}^{\delta^{\prime}}\right), L^{2}\left(\mathbb{R}_{+}, x_{n}^{\delta}\right)\right)_{\theta, q} \subseteq L^{q}\left(\mathbb{R}_{+}\right), \\
\left(H_{2}^{-\delta^{\prime}}\left(\mathbb{R}_{+}\right), H_{2}^{-\delta}\left(\mathbb{R}_{+}\right)\right)_{\theta, q} \supseteq L^{q}\left(\mathbb{R}_{+}\right),
\end{gathered}
$$


where $0 \leq \delta^{\prime}<\frac{1}{q}-\frac{1}{2}<\delta<\frac{1}{2}, \theta=\left(\frac{1}{q}-\frac{1}{2}-\delta^{\prime}\right) /\left(\delta-\delta^{\prime}\right)$, cf.e.g., [11, Lemma 2.1], and $(., .)_{\theta, q}$ denotes the real interpolation functor. This implies the statement in this case. If $2 \leq q<\infty$, then one uses instead

$$
\begin{aligned}
& \left(L^{2}\left(\mathbb{R}_{+}, x_{n}^{-\delta^{\prime}}\right), L^{2}\left(\mathbb{R}_{+}, x_{n}^{-\delta}\right)\right)_{\theta, q} \supseteq L^{q}\left(\mathbb{R}_{+}\right), \\
& \left(H_{2}^{\delta^{\prime}}\left(\mathbb{R}_{+}\right), H_{2}^{\delta}\left(\mathbb{R}_{+}\right)\right)_{\theta, q} \subseteq L^{q}\left(\mathbb{R}_{+}\right),
\end{aligned}
$$

where $0 \leq \delta^{\prime}<\frac{1}{2}-\frac{1}{q}<\delta<\frac{1}{2}$, and $\theta=\left(\frac{1}{2}-\frac{1}{q}-\delta^{\prime}\right) /\left(\delta-\delta^{\prime}\right)$, cf. e.g., [11, Lemma 2.1] again. This finishes the proof.

For the pseudodifferential operator part $\underline{p}_{\lambda}\left(x, D_{x}\right)$ we can apply:

Lemma 13 Let $1<q<\infty, R>0$, and $\delta \in(0, \pi)$. Then $\underline{p}_{\lambda}(x, \xi)=(\lambda+$ $\left.\underline{v}(x)|A(x) \xi|^{2}\right)^{-1}, x \in \mathbb{R}^{d}, \xi \in \mathbb{R}$, with $A, A^{-1} \in C^{\tau}\left(\mathbb{R}^{d}\right)^{d \times d}, \underline{\underline{v}}, \underline{v}^{-1} \in C^{\tau}\left(\mathbb{R}^{d}\right)$ satisfies

$$
\left\|\int_{\Gamma_{R}} h(-\lambda) D_{\xi}^{\alpha} \underline{p}_{\lambda}(., \xi) d \lambda\right\|_{C^{\tau}} \leq C_{\delta, R, \alpha}\|h\|_{\infty}\langle\xi\rangle^{-|\alpha|}
$$

uniformly in $\xi \in \mathbb{R}^{d}$, for all $\alpha \in \mathbb{N}_{0}^{d}$ and $h \in H(\delta)$.

Proof The proof is literally the same as in [11, Lemma 5.14] just replacing $C^{0,1}$-norms by $C^{\tau}$-norms.

Now we are in the position to prove the following main step in the proof of Theorem 4:

Theorem 9 Let $1<q<\infty, a \in C^{\tau}\left(\mathbb{R}^{d}\right)$ with $\tau>0,0<\delta<\pi, \lambda \in \Sigma_{\delta}$, and let $\underline{r}_{j, \lambda}^{r}\left(D_{x}, x\right)$ be as above. Then $\underline{r}_{j, \lambda}^{r}\left(D_{x}, x\right)$ extends to a bounded operator $\underline{r}_{j, \lambda}^{r}\left(D_{x}, x\right): L^{q}\left(\mathbb{R}_{+}^{d}\right)^{d} \rightarrow W_{q}^{2}\left(\mathbb{R}_{+}^{d}\right)^{d}$ and

$$
\begin{aligned}
& (\lambda-\underline{v} \Delta) \underline{r}_{j, \lambda}^{r}\left(D_{x}, x\right) f+\underline{\nabla}_{j, \lambda}\left(D_{x}, x^{\prime}\right) f=f+S_{j, \lambda} f \quad \text { in } \mathbb{R}_{+}^{d}, \\
& \underline{t}_{j}^{\prime}\left(x^{\prime}, D_{x}\right) \underline{r}_{j, \lambda}^{r}\left(D_{x}, x\right) f=S_{j, \lambda}^{\prime} f \quad \text { on } \partial \mathbb{R}_{+}^{d}
\end{aligned}
$$

for every $f \in L^{q}\left(\mathbb{R}_{\gamma}^{d}\right)^{d}$ where $\underline{g}_{j, \lambda}\left(D_{x}, x^{\prime}\right)$ is a singular Green operator of order -1 , class 0 , and regularity $\frac{1}{2}$. Moreover,

$$
\begin{aligned}
& \left(\underline{\nabla}_{j, \lambda}\left(D_{x}, x^{\prime}\right) f, a \underline{\nabla} \varphi\right)_{\mathbb{R}_{+}^{d}} \\
& =\left(\underline{v}(\underline{\Delta}-\underline{\nabla \operatorname{div}}) \underline{r}_{j, \lambda}^{r}\left(D_{x}, x\right) f, a \underline{\nabla} \varphi\right)_{\mathbb{R}_{+}^{d}}+\left\langle S_{j, \lambda}^{\prime \prime} f, \varphi\right\rangle_{W_{q, 0}^{-1}, W_{q^{\prime}}^{1}}
\end{aligned}
$$

for all $\varphi \in W_{q^{\prime}}^{1}\left(\mathbb{R}_{+}^{d}\right)$ with $\left.\varphi\right|_{x_{d}=0}=0$ if $j=1$,

$$
\left.\underline{g}_{1, \lambda}\left(D_{x}, x^{\prime}\right) f\right|_{\partial \mathbb{R}_{+}^{d}}=\underline{t}_{1}^{r}\left(x^{\prime}, D_{x}\right) \underline{r}_{1, \lambda}^{r}\left(D_{x}, x\right) f+S_{\lambda}^{\prime \prime \prime} f \quad \text { on } \partial \mathbb{R}_{+}^{d},
$$


and

$$
\begin{aligned}
& \left\|S_{j, \lambda} f\right\|_{L^{q}\left(\mathbb{R}_{+}^{d}\right)}+\left\|S_{j, \lambda}^{\prime} f\right\|_{W_{q}^{2-j-\frac{1}{q}}\left(\mathbb{R}^{d-1}\right)} \leq C_{q, \delta}\langle\lambda\rangle^{-\varepsilon}\|f\|_{L^{q}\left(\mathbb{R}_{+}^{d}\right)}, \\
& \left\|S_{j, \lambda}^{\prime \prime} f\right\|_{\left.W_{q, 0}^{-1}\left(\mathbb{R}_{+}^{d}\right)\right)}+\left\|S_{\lambda}^{\prime \prime \prime} f\right\|_{W_{q}^{1-\frac{1}{q}}\left(\mathbb{R}^{d-1}\right)} \leq C_{q, \delta}\langle\lambda\rangle^{-\varepsilon}\|f\|_{L^{q}\left(\mathbb{R}_{+}^{d}\right)}, \\
& \langle\lambda\rangle^{\frac{1}{2}\left(2-j-\frac{1}{q}\right)}\left\|S_{j, \lambda}^{\prime} f\right\|_{L^{q}\left(\mathbb{R}^{d-1}\right)} \leq C_{q, \delta}\langle\lambda\rangle^{-\varepsilon}\|f\|_{L^{q}\left(\mathbb{R}_{+}^{d}\right)}
\end{aligned}
$$

uniformly in $\lambda \in \Sigma_{\delta}, f \in L^{q}\left(\mathbb{R}_{+}^{d}\right)^{d}$ for some $\varepsilon>0$. Finally,

$$
\begin{aligned}
&\langle\lambda\rangle\left\|\underline{r}_{j, \lambda}^{r}\left(D_{x}, x\right)\right\|_{\mathcal{L}\left(L^{q}\left(\mathbb{R}_{+}^{d}\right)\right)}+\left\|\nabla^{2} \underline{r}_{j, \lambda}^{r}\left(D_{x}, x\right)\right\|_{\mathcal{L}\left(L^{q}\left(\mathbb{R}_{+}^{d}\right)\right)} \leq C_{q, \delta}, \\
&\langle\lambda\rangle^{\frac{1}{2}}\left\|\underline{g}_{j, \lambda}^{r}\left(D_{x}, x^{\prime}\right)\right\|_{\mathcal{L}\left(L^{q}\left(\mathbb{R}_{+}^{d}\right)\right)}+\left\|\nabla \underline{g}_{j, \lambda}^{r}\left(D_{x}, x^{\prime}\right)\right\|_{\mathcal{L}\left(L^{q}\left(\mathbb{R}_{+}^{d}\right)\right)} \leq C_{q, \delta}
\end{aligned}
$$

uniformly in $\lambda \in \Sigma_{\delta},|\lambda| \geq R_{0}$, where $R_{0}$ is as in Theorem 7 .

Proof First of all, because of Corollary 3, Theorem 5, Theorem 6.2, and Remarks 4 and 5 ,

$$
\underline{r}_{j, \lambda}^{r}\left(D_{x}, x\right): L^{q}\left(\mathbb{R}_{+}^{d}\right)^{d} \rightarrow W_{q}^{2}\left(\mathbb{R}_{+}^{d}\right)^{d}
$$

with operator norm uniformly bounded in $\lambda \in \Sigma_{\delta},|\lambda| \geq R_{0}, \delta \in(0, \pi)$. Considering $\underline{p}_{\lambda}(x, \xi), \underline{\tilde{g}}_{j, \lambda}^{r}\left(x^{\prime}, \xi^{\prime}, x_{n}, y_{n}\right)$ as symbol(-kernels) of order 0 with symbol semi-norms bounded by $C_{\delta}(1+|\lambda|)^{-1}$, cf. Remark 4 and Remark 5.1, we conclude $\left\|R_{j, \lambda}\right\|_{\mathcal{L}\left(L^{q}\left(\mathbb{R}_{+}^{d}\right)\right)} \leq C_{\delta}(1+|\lambda|)^{-1}$. Hence (5.32) holds.

In order to show (5.25), we calculate

$$
\begin{aligned}
(\lambda & \left.-\underline{v}_{\underline{\Delta} r_{j, \lambda}^{r}}\left(D_{x}, x\right) f\right) \\
& =(\lambda-\underline{v} \underline{\Delta}) \underline{p}_{\lambda}\left(D_{x}, x\right)_{+} f+(\lambda-\underline{v} \underline{\Delta}) \underline{g}_{j, \lambda}^{r}\left(D_{x}, x^{\prime}\right) f \\
& =\mathrm{OP}\left(\underline{q}_{\lambda}(y, \xi) \underline{p}_{\lambda}(y, \xi)\right)+\mathrm{OP}^{\prime}\left(\mathrm{OP}_{d}\left(\underline{q}_{\lambda}\left(y^{\prime}, 0, \xi\right)\right)_{+} \underline{g}_{j, \lambda}^{r}\left(y^{\prime}, \xi^{\prime}, D_{d}\right)\right)+\widetilde{S}_{j, \lambda} f \\
& =f+\mathrm{OP}^{\prime}\left(\underline{q}_{\lambda}\left(y^{\prime}, 0, \xi^{\prime}, D_{d}\right)_{+} \underline{g}_{j, \lambda}^{r}\left(y^{\prime}, \xi^{\prime}, D_{d}\right)\right) f+\widetilde{S}_{j, \lambda} f
\end{aligned}
$$

where $\underline{q}_{\lambda}(x, \xi)=\lambda+\underline{v}(x)|A(x) \xi|^{2}$ and

$$
\begin{aligned}
\left\|\widetilde{S}_{j, \lambda}\right\|_{\mathcal{L}\left(L^{q}\left(\mathbb{R}_{+}^{d}\right)\right)} & \leq C\left(\left|\underline{p}_{\lambda}\right|_{k}^{(-2+\varepsilon)}+\left|\underline{g}_{j, \lambda}^{r}\right|_{k}^{(-3+\varepsilon)}\right) \\
& \leq C\langle\lambda\rangle^{-\frac{\varepsilon}{2}}\left(\left|\underline{p}_{\lambda}\right|_{k}^{(-2,0)}+\left|\underline{g}_{j, \lambda}^{r}\right|_{k}^{(-3,0)}\right)
\end{aligned}
$$

uniformly in $\lambda \in \Sigma_{\delta},|\lambda| \geq R_{0}$, for some $\varepsilon>0$ and $k \in \mathbb{N}$ because of Lemma 10 with $d_{1}=2$, Remark 4, and Remark 5.1. Next

$$
\underline{q}_{\lambda}\left(y^{\prime}, \xi^{\prime}, D_{d}\right)_{+} \underline{g}_{j, \lambda}^{r}\left(y^{\prime}, \xi^{\prime}, D_{d}\right)=-U\left(y^{\prime}\right)^{T}\left(\begin{array}{c}
A^{\prime}\left(y^{\prime}\right) \xi^{\prime} \\
\partial_{d}
\end{array}\right) \underline{g}_{j, \lambda}\left(y^{\prime}, \xi^{\prime}, D_{d}\right),
$$


where

$$
\underline{g}_{j, \lambda}\left(y^{\prime}, \xi^{\prime}, D_{d}\right)=\underline{v}^{\frac{1}{2}}\left(y^{\prime}\right) g_{j, \lambda}\left(\underline{v}^{\frac{1}{2}}\left(y^{\prime}\right) A^{\prime}\left(y^{\prime}\right) \xi^{\prime}, \underline{v}^{\frac{1}{2}}\left(y^{\prime}\right) D_{d}\right) U\left(y^{\prime}\right)
$$

and $g_{j, \lambda}\left(\xi^{\prime}, D_{d}\right)$ is as in (5.19). Hence

$$
\mathrm{OP}^{\prime}\left(\underline{q}_{\lambda}\left(y^{\prime}, \xi^{\prime}, D_{d}\right)_{+} \underline{g}_{j, \lambda}^{r}\left(y^{\prime}, \xi^{\prime}, D_{d}\right)\right) f=-\underline{\nabla}_{j, \lambda}\left(D_{x}, x^{\prime}\right) f+\widetilde{S}_{j, \lambda}^{\prime} f,
$$

where $\left\|{\widetilde{S_{j, \lambda}}}_{j}^{\prime}\right\|_{\mathcal{L}\left(L^{q}\left(\mathbb{R}_{+}^{d}\right)\right)} \leq C\langle\lambda\rangle^{-\varepsilon}$ uniformly in $\lambda \in \Sigma_{\delta},|\lambda| \geq R_{0}$ for some $\varepsilon>0$ because of Lemma 10 and Remark 5 as before. Thus (5.25) holds true with $S_{j, \lambda}=$ $\widetilde{S}_{j, \lambda}+\widetilde{S}_{j, \lambda}^{\prime}$

Since $\underline{g}_{j, \lambda}\left(D_{x}, x^{\prime}\right)$ is a parameter-dependent singular Green operator of order -1 , class 0 , and regularity $\frac{1}{2}$, we obtain $(5.33)$ by the same arguments as for (5.32). In order to prove (5.27), we derive for all $\varphi \in W_{q^{\prime}}^{1}\left(\mathbb{R}_{+}^{d}\right)$ with $\left.\varphi\right|_{x_{d}=0}=0$ if $j=1$ that

$$
\begin{aligned}
\left(\underline{\nabla}_{j, \lambda}\left(D_{x}, x^{\prime}\right) f, a \underline{\nabla} \varphi\right)_{\mathbb{R}_{+}^{d}}=\left(a A^{T} A \mathrm{OP}\left(i \xi \underline{g}_{j, \lambda}\left(\xi, y^{\prime}\right)\right) f, \nabla \varphi\right)_{\mathbb{R}_{+}^{d}} \\
=\left(\mathrm{OP}^{\prime}\left(a\left(y^{\prime}\right) A\left(y^{\prime}\right)^{T} A\left(y^{\prime}\right) \mathrm{OP}_{d}(i \xi) \underline{g}_{j, \lambda}\left(\xi^{\prime}, y^{\prime}, D_{d}\right)\right) f, \nabla \varphi\right)_{\mathbb{R}_{+}^{d}}+\left(\widetilde{S}_{j, \lambda} f, \nabla \varphi\right)_{\mathbb{R}_{+}^{d}} \\
=-\left(\left.\operatorname{OP}^{\prime}\left(a\left(y^{\prime}\right) \partial_{d} \underline{g}_{j, \lambda}\left(\xi^{\prime}, y^{\prime}, D_{d}\right)\right) f\right|_{x_{d}=0},\left.\varphi\right|_{x_{d}=0}\right)_{\mathbb{R}^{d-1}} \\
\quad-\left(\operatorname{div} \mathrm{OP}^{\prime}\left(a\left(y^{\prime}\right) A\left(y^{\prime}\right)^{T} A\left(y^{\prime}\right) \mathrm{OP}_{d}(i \xi) \underline{g}_{j, \lambda}\left(\xi^{\prime}, y^{\prime}, D_{d}\right)\right) f, \varphi\right)_{\mathbb{R}_{+}^{d}}+\left(\widetilde{S}_{j, \lambda} f, \nabla \varphi\right)_{\mathbb{R}_{+}^{d}}
\end{aligned}
$$

where $\left\|\widetilde{S}_{j, \lambda} f\right\|_{L^{q}\left(\mathbb{R}_{+}^{d}\right)} \leq C\langle\lambda\rangle^{-\varepsilon}\|f\|_{L^{q}\left(\mathbb{R}_{+}^{d}\right)}$ for some $\varepsilon>0$ because of Lemma 10 with $d_{1}=0$. Moreover,

$$
\begin{gathered}
-\operatorname{div} \mathrm{OP}^{\prime}\left(a\left(y^{\prime}\right) A\left(y^{\prime}\right)^{T} A\left(y^{\prime}\right) \mathrm{OP}_{d}(i \xi) \underline{g}_{j, \lambda}\left(\xi^{\prime}, y^{\prime}, D_{d}\right)\right) f \\
=\operatorname{OP}^{\prime}\left(a\left(y^{\prime}\right)\left(\left|A^{\prime}\left(y^{\prime}\right) \xi^{\prime}\right|^{2}-\partial_{d}^{2}\right) \underline{g}_{j, \lambda}\left(\xi^{\prime}, y^{\prime}, D_{d}\right)\right)=0
\end{gathered}
$$

due to (5.21) and (5.34). Furthermore, if $j=0$, then

$$
\begin{aligned}
\mathrm{OP}^{\prime} & \left.\left(a\left(y^{\prime}\right) \partial_{d} \underline{g}_{0, \lambda}\left(\xi^{\prime}, y^{\prime}, D_{d}\right)\right) f\right|_{x_{d}=0} \\
= & \mathrm{OP}^{\prime}\left(a\left(y^{\prime}\right) \underline{t}_{0}^{r}\left(\xi^{\prime}, y^{\prime}, D_{d}\right) \underline{r}_{0, \lambda}^{r}\left(\xi^{\prime}, y^{\prime}, D_{d}\right)\right) f \\
= & e_{d} \cdot \gamma_{0} \mathrm{OP}\left(a(y)\left(|A(y) \xi|^{2}-(A(y) \xi)(A(y) \xi)^{T}\right) \underline{p}_{\lambda}\left(\xi^{\prime}, y\right) U(y)\right)_{+} f+S_{0, \lambda}^{\prime} f \\
& +e_{d} \cdot \gamma_{0} \mathrm{OP}^{\prime}\left(a\left(y^{\prime}\right) \mathrm{OP}_{d}\left(\left|A\left(y^{\prime}\right) \xi\right|^{2}-\left(A\left(y^{\prime}\right) \xi\right)\left(A\left(y^{\prime}\right) \xi\right)^{T}\right) U\left(y^{\prime}\right) \underline{g}_{0, \lambda}^{r}\left(\xi^{\prime}, y^{\prime}, D_{d}\right)\right) f
\end{aligned}
$$

due to (5.20) and (5.34), where

$$
\begin{aligned}
& \mathrm{OP}^{\prime}\left(t\left(y, \xi^{\prime}, D_{d}\right)\right) f \\
& \equiv e_{d} \cdot \gamma_{0} \mathrm{OP}\left(a(y)\left(|A(y) \xi|^{2}-(A(y) \xi)(A(y) \xi)^{T}\right) \underline{p}_{\lambda}(\xi, y) U(y)\right)_{+} f \\
& =\mathrm{OP}^{\prime}\left(t\left(y^{\prime}, 0, \xi^{\prime}, D_{d}\right)\right) f+S_{j, \lambda}^{\prime} f
\end{aligned}
$$


Here $\operatorname{OP}^{\prime}\left(t\left(y, \xi^{\prime}, D_{d}\right)\right)$ is a trace operator of order 0 and class 0 since $e_{d} \cdot\left(|A(y) \xi|^{2}-\right.$ $\left.(A(y) \xi) A(y) \xi)^{T}\right) \underline{p}_{\lambda}(\xi, y)=O\left(\left\langle\xi_{d}\right\rangle^{-1}\right)$ w.r.t. $\xi_{d}$, cf. [37, Proposition 2.2.2]. Therefore Lemma 11 implies $\left\|S_{j, \lambda}^{\prime} f\right\|_{B_{q q}^{-\frac{1}{q}}\left(\mathbb{R}^{d-1}\right)} \leq C\langle\lambda\rangle^{-\varepsilon}\|f\|_{L^{q}\left(\mathbb{R}_{+}^{d}\right)}$ for some $\varepsilon>0$. Finally, if $M(y, \xi)=\left(|A(y) \xi|^{2}-(A(y) \xi)(A(y) \xi)^{T}\right) U(y)$, then

$$
\begin{aligned}
\left(e_{d}\right. & \left.\cdot \gamma_{0} \operatorname{OP}\left(a(y) M(y, \xi) \underline{p}_{\lambda}\left(\xi^{\prime}, y\right)\right)_{+} f,\left.\varphi\right|_{x_{d}=0}\right)_{\mathbb{R}^{d-1}} \\
& =-\left(\operatorname{OP}\left(a(y) M(x, \xi) \underline{p}_{\lambda}\left(\xi^{\prime}, y\right)\right)_{+} f, \nabla \varphi\right)_{\mathbb{R}_{+}^{d}} \\
& =-\left(a(\underline{\Delta}-\underline{\nabla \operatorname{div}}) \underline{p}_{\lambda}\left(D_{x}, x\right) f, \underline{\nabla} \varphi\right)_{\mathbb{R}_{+}^{d}}+\left(\widetilde{S}_{j, \lambda}^{\prime \prime} f, \nabla \varphi\right)_{\mathbb{R}_{+}^{d}}
\end{aligned}
$$

and

$$
\begin{aligned}
\left(e_{d}\right. & \left.\cdot \gamma_{0} \mathrm{OP}^{\prime}\left(a\left(y^{\prime}\right) M\left(y^{\prime}, \xi^{\prime}, D_{d}\right) \underline{g}_{j, \lambda}^{r}\left(\xi^{\prime}, y^{\prime}, D_{d}\right)\right) f,\left.\varphi\right|_{x_{d}=0}\right)_{\mathbb{R}^{d-1}} \\
& =-\left(\mathrm{OP}^{\prime}\left(a\left(y^{\prime}\right) M\left(y^{\prime}, \xi^{\prime}, D_{d}\right) \underline{g}_{j, \lambda}^{r}\left(\xi^{\prime}, y^{\prime}, D_{d}\right)\right) f, \nabla \varphi\right)_{\mathbb{R}_{+}^{d}} \\
& =-\left(a(\underline{\Delta}-\underline{\nabla \operatorname{div}}) \underline{g}_{j, \lambda}^{r}\left(D_{x}, x^{\prime}\right) f, \underline{\nabla} \varphi\right)_{\mathbb{R}_{+}^{d}}+\left(\widehat{S}_{j, \lambda}^{\prime \prime} f, \nabla \varphi\right)_{\mathbb{R}_{+}^{d}}
\end{aligned}
$$

since

$$
\begin{aligned}
& \operatorname{div} \operatorname{OP}\left(a(y) M(y, \xi) \underline{p}_{\lambda}\left(\xi^{\prime}, y\right)\right) \\
& \quad=\operatorname{OP}\left(a(y) i \xi \cdot\left(|A(y) \xi|^{2}-(A(y) \xi)(A(y) \xi)^{T}\right) U(y) \underline{p}_{\lambda}\left(\xi^{\prime}, y\right)\right)=0 \quad \text { and } \\
& \operatorname{div} \operatorname{OP}^{\prime}\left(a\left(y^{\prime}\right) M\left(y^{\prime}, \xi^{\prime}, D_{d}\right) \underline{g}_{j, \lambda}^{r}\left(\xi^{\prime}, y^{\prime}, D_{d}\right)\right) \\
& =\mathrm{OP}^{\prime}\left(a\left(y^{\prime}\right) \mathrm{OP}_{d}\left(i \xi \cdot\left(\left|A\left(y^{\prime}\right) \xi\right|^{2}-\left(A\left(y^{\prime}\right) \xi\right)\left(A\left(y^{\prime}\right) \xi\right)^{T} U\left(y^{\prime}\right)\right)\right) \underline{g}_{j, \lambda}^{r}\left(\xi^{\prime}, y^{\prime}, D_{d}\right)\right) \\
& =0 .
\end{aligned}
$$

Here $\left\|S_{j, \lambda}^{\prime \prime} f\right\|_{L^{q}\left(\mathbb{R}_{+}^{d}\right)} \leq C\langle\lambda\rangle^{-\varepsilon}\|f\|_{L^{q}\left(\mathbb{R}_{+}^{d}\right)}$ because of Lemma 10 and Remark 5 again. Furthermore, if $j=1$,

$$
\begin{aligned}
& \left.\underline{g}_{1, \lambda}^{r}\left(D_{x}, x^{\prime}\right)\right|_{x_{d}=0} \\
& \quad=\mathrm{OP}^{\prime}\left(\underline{t}_{1}^{r}\left(y^{\prime}, \xi^{\prime}, D_{d}\right) \underline{r}_{j, \lambda}^{r}\left(y^{\prime}, \xi^{\prime}, D_{d}\right)\right) \\
& \quad=\mathrm{OP}^{\prime}\left(\underline{t}_{1}^{r}\left(y^{\prime}, \xi^{\prime}, D_{d}\right) \underline{p}_{\lambda}\left(y^{\prime}, 0, \xi^{\prime}, D_{d}\right)_{+}\right)+\mathrm{OP}^{\prime}\left(\underline{t}_{1}^{r}\left(y^{\prime}, \xi^{\prime}, D_{d}\right) \underline{g}_{j, \lambda}^{r}\left(y^{\prime}, \xi^{\prime}, D_{d}\right)\right) \\
& \quad=\underline{t}_{1}^{r}\left(x^{\prime}, D_{x}\right)\left(p_{\lambda}\left(D_{x}, x\right)_{+}+g_{1, \lambda}^{r}\left(D_{x}, x^{\prime}\right)\right)+S_{\lambda}^{\prime \prime \prime}
\end{aligned}
$$

due to (5.20), where $S_{\lambda}^{\prime \prime \prime}$ satisfies (5.30) because of Lemma 10 and Remark 5 again.

Finally,

$$
\begin{gathered}
\gamma_{0} \underline{r}_{0, \lambda}^{r}\left(D_{x}, x\right)=\mathrm{OP}^{\prime}\left(\gamma_{0} \underline{r}_{1, \lambda}^{r}\left(y^{\prime}, \xi^{\prime}, D_{d}\right)\right)+S_{0, \lambda}^{\prime}=S_{0, \lambda}^{\prime} \\
\underline{t}_{1}^{\prime}\left(x^{\prime}, D_{x}\right) \underline{r}_{1, \lambda}^{r}\left(D_{x}, x\right)=\operatorname{OP}^{\prime}\left(\underline{t}_{1}^{\prime}\left(y^{\prime}, \xi^{\prime}, D_{d}\right) \underline{r}_{1, \lambda}^{r}\left(y^{\prime}, \xi^{\prime}, D_{d}\right)\right)+S_{1, \lambda}^{\prime}=S_{1, \lambda}^{\prime}
\end{gathered}
$$


where $S_{j, \lambda}^{\prime}$ satisfies the estimate in (5.29) because of Lemma 10 and Remark 5 once more. Using the $L^{q}\left(\mathbb{R}^{d-1}\right)$-estimates stated in Lemma 10, one derives (5.31), where we note that

$$
\begin{aligned}
& \left|\underline{p}_{\lambda}\right|_{k}^{\left(-j-\frac{1}{q}+\varepsilon\right)}+\left|\underline{g}_{j, \lambda}^{r}\right|_{k}^{\left(-1-j-\frac{1}{q}+\varepsilon\right)} \\
& \quad \leq C(1+|\lambda|)^{-\frac{1}{2}\left(2-j+\frac{1}{q}+\varepsilon\right)}\left(|\underline{q}|_{k}^{(-2,0)}+\left|\underline{g}_{j}^{r}\right|_{k}^{(-3,0)}\right) .
\end{aligned}
$$

This finishes the proof.

\subsection{Proof of Theorem 4}

Let $\mathbb{R}_{\gamma}^{d}$ be a bent half-space as in the assumptions of Theorem 4 . Then we define

$$
R_{j, \lambda}:=R_{j, \lambda}^{\prime}-E_{j} T_{j}^{\prime} R_{j, \lambda}^{\prime}, \quad \text { where } R_{j, \lambda}^{\prime}:=F^{*,-1} \mathrm{OP}^{\prime}\left(\underline{r}_{j, \lambda}^{r}\left(\underline{y}, \underline{\xi}^{\prime}, D_{d}\right)\right) F^{*}
$$

as parametrix for the reduced Stokes equations in $\mathbb{R}_{\gamma}^{d}$, where $\underline{r}_{j, \lambda}^{r}$ is defined in (5.24) (extended for $|\lambda| \leq R_{0}$ suitably) and $E_{j}$ is the extension operator due to Lemma 7. Then (5.2)-(5.3) hold. Because of (5.32) and Corollary 1, $R_{j, \lambda}^{\prime}: L^{q}\left(\mathbb{R}_{\gamma}^{d}\right)^{d} \rightarrow W_{q}^{2}\left(\mathbb{R}_{\gamma}^{d}\right)^{d}$ with operator norm uniformly bounded in $\lambda \in \Sigma_{\delta} \cup\{0\}$ for every $\delta \in(0, \pi)$ and $\left\|R_{j, \lambda}^{\prime}\right\|_{\mathcal{L}\left(L^{q}\left(\mathbb{R}_{\gamma}^{d}\right)\right)} \leq C_{\delta}(1+|\lambda|)^{-1}, \lambda \in \Sigma_{\delta}$. Therefore Lemma 7, (5.26), (5.29), (5.30), and Lemma 6 imply

$$
\left\|\left(\langle\lambda\rangle E_{j} T_{j}^{\prime} R_{j, \lambda}^{\prime} f, \nabla^{2} E_{j} T_{j}^{\prime} R_{j, \lambda}^{\prime} f\right)\right\|_{L^{q}\left(\mathbb{R}_{\gamma}^{d}\right)} \leq C\langle\lambda\rangle^{-\varepsilon}\|f\|_{L^{q}\left(\mathbb{R}_{\gamma}^{d}\right)} .
$$

Hence (5.8) holds. Moreover, because of Theorem 8 and Lemma 13 together with Theorem 5, (5.10) holds, where we note that

$$
\left\|\int_{\Gamma_{R}} h(-\lambda) E_{j} T_{j}^{\prime} R_{j, \lambda}^{\prime} d \lambda\right\|_{\mathcal{L}\left(L^{q}\left(\mathbb{R}_{\gamma}^{d}\right)\right)} \leq C_{\delta}\|h\|_{\infty} \quad \text { for all } h \in H(\delta)
$$

since $\left\|E_{j} T_{j}^{\prime} R_{j, \lambda}^{\prime} f\right\|_{L^{q}\left(\mathbb{R}_{\gamma}^{d}\right)} \leq C\langle\lambda\rangle^{-1-\varepsilon}\|f\|_{L^{q}\left(\mathbb{R}_{\gamma}^{d}\right)}$ for some $\varepsilon>0$.

Due to Lemma 6 and (5.8),

$$
(\lambda-\operatorname{div}(v \nabla \cdot)) R_{j, \lambda}=F^{*,-1} \underline{q}_{\lambda}\left(x, D_{x}\right) \mathrm{OP}^{\prime}\left(\underline{r}_{j, \lambda}^{r}\left(y, \xi^{\prime}, D_{d}\right)\right) F^{*}+\widetilde{S}_{\lambda}^{\prime}
$$

where $\underline{q}_{\lambda}(x, \xi)=\lambda+\underline{v}(x)|A(x) \xi|^{2}$ and $\widetilde{S}_{\lambda}^{\prime}=O\left((1+|\lambda|)^{-\varepsilon}\right)$ in $\mathcal{L}\left(L^{q}\left(\mathbb{R}_{\gamma}^{d}\right)\right)$. Because of (5.25) and Lemma 6 again, we conclude further that

$$
\begin{aligned}
(\lambda-\operatorname{div}(\nu \nabla \cdot)) R_{j, \lambda}^{\prime} & \left.=I-F^{*,-1} \underline{\nabla}_{j, \lambda}\left(D_{x}, x^{\prime}\right)\right) F^{*}+\widetilde{S}_{\lambda} \\
& =I-\nabla G_{j, \lambda}+\widetilde{S}_{\lambda}
\end{aligned}
$$


for some $\widetilde{S}_{\lambda}=O\left((1+|\lambda|)^{-\varepsilon}\right)$ in $\mathcal{L}\left(L^{q}\left(\mathbb{R}_{\gamma}^{d}\right)\right)$ where $\left.G_{j, \lambda}=F^{*,-1} \underline{g}_{j, \lambda}\left(D_{x}, x^{\prime}\right)\right) F^{*}$. Combining this with (5.35), we obtain (5.1) together with the estimate of $S_{j, \lambda}$.

It remains to show (5.4)-(5.5). Using Lemma 6 and (5.27) with $a=\operatorname{det} \nabla F(x)$, we obtain

$$
\begin{aligned}
\left(\nabla G_{j, \lambda} f, \nabla \varphi\right)_{\mathbb{R}_{\gamma}^{d}}=\left(\underline{\nabla} g_{j, \lambda}\left(D_{x}, x^{\prime}\right) F^{*} f, \operatorname{det} \nabla F(x) \underline{\nabla} F^{*} \varphi\right)_{\mathbb{R}_{+}^{d}} \\
=\left(\underline{v}(\underline{\Delta}-\underline{\nabla \operatorname{div}}) \underline{r}_{j, \lambda}\left(D_{x}, x\right) F^{*} f, a \underline{\nabla} F^{*} \varphi\right)_{\mathbb{R}_{+}^{d}}+\left\langle S_{j, \lambda}^{\prime \prime} F^{*} f, F^{*} \varphi\right\rangle_{W_{q, 0}^{-1}, W_{q^{\prime}}^{1}} \\
=\left(\nu(\Delta-\nabla \operatorname{div}) R_{j, \lambda} f, \varphi\right)_{\mathbb{R}_{\gamma}^{d}}+\left(\underline{\nu} R_{1} \underline{r}_{j, \lambda}\left(D_{x}, x\right) F^{*} f, a \underline{\nabla} F^{*} \varphi\right)_{\mathbb{R}_{+}^{d}} \\
\quad+\left\langle S_{j, \lambda}^{\prime \prime} F^{*} f, F^{*} \varphi\right\rangle \\
\equiv\left(v(\Delta-\nabla \operatorname{div}) R_{j, \lambda} f, \varphi\right)_{\mathbb{R}_{\gamma}^{d}}+\left\langle S_{j, \lambda}^{\prime} f, \varphi\right\rangle_{W_{q, 0}^{-1}, W_{q^{\prime}}^{1}}
\end{aligned}
$$

for all $\varphi \in W_{q^{\prime}}^{1}\left(\mathbb{R}_{\gamma}^{d}\right)$ with $\left.\varphi\right|_{\partial \mathbb{R}_{\gamma}^{d}}=0$ if $j=0$, where $R_{1}$ is a differential operator of order 1 with $L^{r_{2}}$-coefficients, $r_{2}>d$. Hence

$$
\begin{aligned}
\left|\left(\underline{\nu} R_{1} \underline{r}_{j, \lambda}\left(D_{x}, x\right) F^{*} f, a \underline{\nabla} F^{*} \varphi\right)_{\mathbb{R}_{+}^{d}}\right| & \leq C\left\|\underline{r}_{j, \lambda}\left(D_{x}, x\right) F^{*} f\right\|_{W_{s}^{1}}\|\nabla \varphi\|_{L^{q^{\prime}}} \\
& \leq C\left\|\underline{r}_{j, \lambda}\left(D_{x}, x\right) F^{*} f\right\|_{W_{q}^{2-2 \varepsilon}}\|\nabla \varphi\|_{L^{q^{\prime}}} \\
& \leq C(1+|\lambda|)^{-\varepsilon}\|f\|_{L^{q}}\|\nabla \varphi\|_{L^{q^{\prime}}}
\end{aligned}
$$

for all $f \in L^{q}\left(\mathbb{R}_{\gamma}^{d}\right), \varphi \in \dot{W}_{q^{\prime}}^{1}\left(\mathbb{R}_{\gamma}^{d}\right)$, and some $\varepsilon>0$, where $\frac{1}{s}=\frac{1}{q}-\frac{1}{r_{2}}>\frac{1}{q}-\frac{1}{d}$. Combining this with (5.30), we have shown the estimates of $S_{j, \lambda}^{\prime}$ stated in (5.6)-(5.7). The identity (5.5) and the estimate of $S_{\lambda}^{\prime \prime}$ follows easily from (5.28), (5.30), and Lemma 6 again. This finishes the proof of Theorem 4.

\section{Estimates of the parametrix}

Now we define the parametrix $R_{\lambda}$ on $\Omega$ by

$$
R_{\lambda} f=\sum_{k=1}^{N} \psi_{k} R_{\gamma_{k}, \lambda} \varphi_{k} f,
$$

where $R_{\gamma_{k}, \lambda}$ denotes the approximate resolvent on $\mathbb{R}_{\gamma_{k}}^{d}$ according to Theorem 4 , where the boundary conditions $(j=0,1)$ are chosen to fit to the boundary conditions on $\partial \Omega \cap U_{k}$. Moreover, we order $\mathbb{R}_{\gamma_{k}}^{d}, k=1, \ldots, N$, such that $U_{k} \cap \Gamma_{1} \neq \varnothing$ and $U_{k} \cap \Gamma_{2}=\emptyset$ for $k=1, \ldots, N_{1}$ as well as $U_{k} \cap \Gamma_{1}=\varnothing$ and $U_{k} \cap \Gamma_{2} \neq \varnothing$ for $k=N_{1}+1, \ldots, N$.

We show that

$$
\begin{aligned}
& (\lambda-\operatorname{div}(\nu \nabla \cdot)+\nabla P) R_{\lambda} f=f+S_{\lambda} f, \\
& \left.R_{\lambda} f\right|_{\Gamma_{1}}=0 \\
& \left.T_{1}^{\prime} R_{\lambda} f\right|_{\Gamma_{2}}=0+S_{\lambda}^{\prime} f,
\end{aligned}
$$


where $P v$ is defined as solution of (1.8)-(1.9) and

$$
\left\|S_{\lambda} f\right\|_{L^{q}(\Omega)}+\left\|S_{\lambda}^{\prime} f\right\|_{W_{q}{ }^{1-\frac{1}{q}}\left(\Gamma_{2}\right)}+\langle\lambda\rangle^{\frac{1}{2}-\frac{1}{2 q}}\left\|S_{\lambda}^{\prime} f\right\|_{L^{q}\left(\Gamma_{2}\right)} \leq C_{\delta, q}\langle\lambda\rangle^{-\varepsilon}\|f\|_{L^{q}(\Omega)}
$$

uniformly in $\lambda \in \Sigma_{\delta}, f \in L^{q}(\Omega)^{d}$. First of all, using Theorem 4, it is easy to check that

$$
(\lambda-\operatorname{div}(\nu \nabla \cdot)) R_{\lambda} f+\nabla G_{\lambda} f=f+S_{\lambda} f
$$

for some $S_{\lambda}$ satisfying the same estimate as in (6.4) and

$$
G_{\lambda} f=\sum_{j=1}^{N} \psi_{j} G_{\gamma_{j}, \lambda} \varphi_{j} f
$$

Here we note that all perturbation terms due to differentiation of the cut-off functions $\varphi_{j}, \psi_{j}$ decay of order at least $\langle\lambda\rangle^{-\frac{1}{2}}$ due to (5.8)-(5.9). Moreover, (6.2)-(6.3) together with the corresponding estimate in (6.4) are proved in a straight forward manner using Theorem 4 again. As mentioned in Remark 3 above, each $G_{\gamma_{j}, \lambda}$ represents the principal of $P R_{\gamma_{j}, \lambda}$ on $\mathbb{R}_{\gamma_{j}}^{d}$. In the same way $G_{\lambda}$ represents the principal part of $P R_{\lambda}$ on $\Omega$. More precisely, we will show that

$$
\left\|\nabla P R_{\lambda} f-\nabla G_{\lambda} f\right\|_{L^{q}(\Omega)} \leq C_{q, \delta}(1+|\lambda|)^{-\varepsilon}\|f\|_{L^{q}(\Omega)}
$$

for all $f \in L^{q}(\Omega)^{d}, \lambda \in \Sigma_{\delta}$ and some $\varepsilon>0$. This is the most important step in the proof of Theorem 1. By duality, it is enough to show that for any $f \in L^{q}(\Omega)^{d}$ and any $u \in L^{q^{\prime}}(\Omega)^{d}$, we have

$$
\left|\left(\nabla P R_{\lambda} f-\nabla G_{\lambda} f, u\right)_{\Omega}\right| \leq C_{q, \delta}(1+|\lambda|)^{-\varepsilon}\|f\|_{q}\|u\|_{q^{\prime}} .
$$

To show this, we use the Helmholtz decomposition for any $u \in L^{q^{\prime}}(\Omega)^{d}$ according to (A2), i.e., $u=u_{0}+\nabla p$ where $u_{0} \in J_{q^{\prime}}(\Omega)$ and $p \in \dot{W}_{q^{\prime}, \Gamma_{2}}^{1}(\Omega)$. Here $p$ can be decomposed by the assumption (A3) as $p=p_{1}+p_{2}$ where $p_{1} \in W_{q^{\prime}, \Gamma_{2}}^{1}(\Omega), p_{2} \in \dot{W}_{q^{\prime}, \Gamma_{2}}^{1}(\Omega)$ with $\nabla p_{2} \in W_{q^{\prime}}^{1}(\Omega)$. Thus we have a decomposition of any $u \in L^{q^{\prime}}(\Omega)^{d}$ such that $u=u_{0}+\nabla p_{1}+\nabla p_{2}$ where $u_{0}, p_{1}, p_{2}$ satisfy the conditions above. We estimate the left-hand side of (6.5) using this decomposition and estimating each term separately, which will be called first, second and third part below.

For the first part, we have

$$
\begin{gathered}
\left(\nabla P\left(\sum_{j=1}^{N} \psi_{j} R_{\gamma_{j}, \lambda} \varphi_{j} f\right)-\nabla\left(\sum_{j=1}^{N} \psi_{j} G_{\gamma_{j}, \lambda} \varphi_{j} f\right), u_{0}\right)_{\Omega} \\
=\left(P\left(\sum_{j=1}^{N} \psi_{j} R_{\gamma_{j}, \lambda} \varphi_{j} f\right)-\sum_{j=1}^{N} \psi_{j} G_{\gamma_{j}, \lambda} \varphi_{j} f, \gamma_{n} u_{0}\right)_{\Gamma_{2}}
\end{gathered}
$$




$$
\begin{aligned}
= & \left(2 \nu \partial_{n}\left(\sum_{j=N_{1}+1}^{N} \psi_{j} R_{\gamma_{j}, \lambda} \varphi_{j} f\right)_{n}\left|\Gamma_{2}-2 \sum_{j=1}^{N} \psi_{j} v\left(\partial_{n} R_{\gamma_{j}, \lambda} \varphi_{j} f\right)_{n}\right|_{\Gamma_{2}}, \gamma_{n} u_{0}\right)_{\Gamma_{2}} \\
& -\left(S_{\lambda}^{\prime \prime} f, \gamma_{n} u_{0}\right)_{\Gamma_{2}} \\
= & \left(\left.2 v \sum_{j=N_{1}+1}^{N}\left(\left(\partial_{n} \psi_{j}\right) R_{\gamma_{j}, \lambda} \varphi_{j} f\right)_{n}\right|_{\partial \mathbb{R}_{\gamma_{j}}^{d}}-S_{\lambda}^{\prime \prime} f, \gamma_{\nu} u_{0}\right)_{\Gamma_{2}}
\end{aligned}
$$

where

$$
\begin{aligned}
& \left\|2 \nu\left(R_{\gamma_{j}, \lambda} \varphi_{j} f\right)_{n}\right\|_{W_{q}^{1-\frac{1}{q}}\left(\partial \mathbb{R}_{\gamma_{j}}^{d}\right)} \leq C_{q, \delta}\langle\lambda\rangle^{-\frac{1}{2}}\|f\|_{q} \text { and } \\
& \left|\left(S_{\lambda}^{\prime \prime} f, \gamma_{\nu} u_{0}\right)_{\Gamma_{2}}\right| \leq C_{q, \delta}\langle\lambda\rangle^{-\varepsilon}\|f\|_{q}\|u\|_{q^{\prime}}
\end{aligned}
$$

because of Theorem 4. Hence the absolute value of (6.6) is estimated from above by $C_{q, \delta}(1+|\lambda|)^{-\varepsilon}\|f\|_{q}\|u\|_{q^{\prime}}$ for some $\varepsilon>0$.

For the second part, we split it further into the Dirichlet and Neumann parts. Here Dirichlet part means that the boundary condition (3.5) is present on that part of the boundary and Neumann part refers to (3.6). For the Dirichlet part, we have

$$
\begin{aligned}
& \left(\nabla P\left(\sum_{j=1}^{N_{1}} \psi_{j} R_{\gamma_{j}, \lambda} \varphi_{j} f\right)-\nabla\left(\sum_{j=1}^{N_{1}} \psi_{j} G_{\gamma_{j}, \lambda} \varphi_{j} f\right), \nabla p_{1}\right)_{\Omega} \\
= & \left(v(\Delta-\nabla \operatorname{div})\left(\sum_{j=1}^{N_{1}} \psi_{j} R_{\gamma_{j}, \lambda} \varphi_{j} f\right), \nabla p_{1}\right)_{\Omega}-\sum_{j=1}^{N_{1}}\left(G_{\gamma_{j}, \lambda} \varphi_{j} f, \nabla \psi_{j} \cdot \nabla p_{1}\right)_{\mathbb{R}_{\gamma_{j}}^{d}} \\
& -\sum_{j=1}^{N_{1}}\left(\nabla G_{\gamma_{j}, \lambda} \varphi_{j} f, \nabla\left(\psi_{j} p_{1}\right)\right)_{\mathbb{R}_{\gamma_{j}}^{d}}+\sum_{j=1}^{N_{1}}\left(\nabla G_{\gamma_{j}, \lambda} \varphi_{j} f, \nabla\left(\psi_{j}\right) p_{1}\right)_{\mathbb{R}_{\gamma_{j}}^{d}} \\
= & \left(v(\Delta-\nabla \operatorname{div})\left(\sum_{j=1}^{N_{1}} \psi_{j} R_{\gamma_{j}, \lambda} \varphi_{j} f\right), \nabla p_{1}\right)_{\Omega} \\
& -\sum_{j=1}^{N_{1}}\left(v(\Delta-\nabla \operatorname{div}) R_{\gamma_{j}, \lambda} \varphi_{j} f, \nabla\left(\psi_{j} p_{1}\right)\right)_{\mathbb{R}_{\gamma_{j}}^{d}}-\sum_{j=1}^{N_{1}}\left\langle S_{0, \lambda}^{\prime} \varphi_{j} f, \psi_{j} p_{1}\right\rangle_{W_{q, 0}^{-1}, W_{q^{\prime}}^{1}} \\
& -\sum_{j=1}^{N_{1}}\left(G_{\gamma_{j}, \lambda} \varphi_{j} f, \nabla \psi_{j} \cdot \nabla p_{1}\right)_{\mathbb{R}_{\gamma_{j}}^{d}}+\sum_{j=1}^{N_{1}}\left(\nabla G_{\gamma_{j}, \lambda} \varphi_{j} f,\left(\nabla \psi_{j}\right) p_{1}\right)_{\mathbb{R}_{\gamma_{j}}^{d}}
\end{aligned}
$$


For the first term of (6.7), we use

$$
\begin{aligned}
& \sum_{j=1}^{N_{1}}\left(\nu(\Delta-\nabla \operatorname{div}) \psi_{j} R_{\gamma_{j}, \lambda} \varphi_{j} f, \nabla p_{1}\right)_{\Omega} \\
& =\sum_{j=1}^{N_{1}}\left(\nu \psi_{j}(\Delta-\nabla \operatorname{div}) R_{\gamma_{j}, \lambda} \varphi_{j} f, \nabla p_{1}\right)_{\mathbb{R}_{\gamma_{j}}^{d}} \\
& \quad+\sum_{j=1}^{N_{1}}\left(\nu\left[\Delta-\nabla \operatorname{div}, \psi_{j}\right] R_{\gamma_{j}, \lambda} \varphi_{j} f, \nabla p_{1}\right)_{\mathbb{R}_{\gamma_{j}}^{d}} \\
& =\sum_{j=1}^{N_{1}}\left(\nu(\Delta-\nabla \operatorname{div}) R_{\gamma_{j}, \lambda} \varphi_{j} f, \nabla\left(\psi_{j} p_{1}\right)\right)_{\mathbb{R}_{\gamma_{j}}^{d}} \\
& \quad-\sum_{j=1}^{N_{1}}\left(\nu(\Delta-\nabla \operatorname{div}) \psi_{j} R_{\gamma_{j}, \lambda} \varphi_{j} f,\left(\nabla \psi_{j}\right) p_{1}\right)_{\mathbb{R}_{\gamma_{j}}^{d}}+\sum_{j=1}^{N_{1}}\left(S_{j} R_{\gamma_{j}, \lambda} \varphi_{j} f, \nabla p_{1}\right)_{\mathbb{R}_{\gamma_{j}}^{d}}
\end{aligned}
$$

where $S_{j}=v\left[\Delta-\nabla \operatorname{div}, \psi_{j}\right]$. If we put (6.8) into (6.7), the first term of (6.8) cancels with the second term of (6.7).

For the estimate of the second term of (6.8), one uses the following estimate,

$$
\begin{aligned}
\left\|v(\Delta-\nabla \operatorname{div}) R_{\gamma_{j}, \lambda} \varphi_{j} f\right\|_{W_{q}^{-\varepsilon}(\Omega)} & \leq C_{q, \delta}\left\|R_{\gamma_{j}, \lambda} \varphi_{j} f\right\|_{W_{q}^{2-\varepsilon}\left(\mathbb{R}_{\gamma_{j}}^{d}\right)} \\
& \leq C_{q, \delta}(1+|\lambda|)^{-\frac{\varepsilon}{2}}\left\|\varphi_{j} f\right\|_{L^{q}\left(\mathbb{R}_{\gamma_{j}}^{d}\right)} \\
& \leq C_{q, \delta}(1+|\lambda|)^{-\frac{\varepsilon}{2}}\|f\|_{L^{q}(\Omega)},
\end{aligned}
$$

where $0<\varepsilon<1$, together with the embedding $W_{q}^{\varepsilon}(\Omega) \hookrightarrow W_{q}^{1}(\Omega)$ for $0<\varepsilon<1$ and the fact that the dual of $W_{q}^{s}(\Omega)$ is $W_{q^{\prime}}^{-s}(\Omega)$ for all $s \in\left(-\frac{1}{q^{\prime}}, \frac{1}{q}\right)$.

Since the commutator $S_{j}=\left[\Delta-\nabla \operatorname{div}, \psi_{j}\right]$ is the differential operator of order 1 , we have for the third term in (6.8) $\left\|S_{j} R_{\gamma_{j}, \lambda} \varphi_{j} f\right\| L^{q}\left(\mathbb{R}_{\gamma_{j}}^{d}\right) \leq C_{q, \delta}(1+|\lambda|)^{-\frac{1}{2}}\|f\|_{q}$.

The remaining terms, which contain the operator $G_{\gamma_{j}, \lambda}$, can be estimated using similar arguments. Hence the absolute value of (6.7) is estimated from above by $C_{q, \delta}(1+|\lambda|)^{-\varepsilon}\|f\|_{q}\|u\|_{q^{\prime}}$ for some $\varepsilon>0$.

For the Neumann part, we have

$$
\begin{aligned}
& \left(\nabla P\left(\sum_{j=N_{1}+1}^{N} \psi_{j} R_{\gamma_{j}, \lambda} \varphi_{j} f\right)-\nabla\left(\sum_{j=N_{1}+1}^{N} \psi_{j} G_{\gamma_{j}, \lambda} \phi_{j} f\right), \nabla p_{1}\right)_{\Omega} \\
& =\left(v(\Delta-\nabla \operatorname{div})\left(\sum_{j=N_{1}+1}^{N} \psi_{j} R_{\gamma_{j}, \lambda} \varphi_{j} f\right), \nabla p_{1}\right)_{\Omega}
\end{aligned}
$$




$$
\begin{aligned}
& -\sum_{j=N_{1}+1}^{N}\left(G_{\gamma_{j}, \lambda} \varphi_{j} f, \nabla \psi_{j} \cdot \nabla p_{1}\right)_{\mathbb{R}_{\gamma_{j}}^{d}} \\
& -\sum_{j=N_{1}+1}^{N}\left(\nabla G_{\gamma_{j}, \lambda} \varphi_{j} f, \nabla\left(\psi_{j} p_{1}\right)\right)_{\mathbb{R}_{\gamma_{j}}^{d}}+\sum_{j=N_{1}+1}^{N}\left(\nabla G_{\gamma_{j}, \lambda} \varphi_{j} f,\left(\nabla \psi_{j}\right) p_{1}\right)_{\mathbb{R}_{\gamma_{j}}^{d}} \\
& =\left(v(\Delta-\nabla \operatorname{div})\left(\sum_{j=N_{1}+1}^{N} \psi_{j} R_{\gamma_{j}, \lambda} \varphi_{j} f\right), \nabla p_{1}\right)_{\Omega} \\
& -\sum_{j=N_{1}+1}^{N}\left(G_{\gamma_{j}, \lambda} \varphi_{j} f, \nabla \psi_{j} \cdot \nabla p_{1}\right)_{\mathbb{R}_{\gamma_{j}}^{d}} \\
& -\sum_{j=N_{1}+1}^{N}\left(v(\Delta-\nabla \operatorname{div}) R_{\gamma_{j}, \lambda} \varphi_{j} f+S_{1, \lambda}^{\prime} \varphi_{j} f, \nabla\left(\psi_{j} p_{1}\right)\right)_{\mathbb{R}_{\gamma_{j}}^{d}} \\
& +\sum_{j=N_{1}+1}^{N}\left(\nabla G_{\gamma_{j}, \lambda} \varphi_{j} f,\left(\nabla \psi_{j}\right) p_{1}\right)_{\mathbb{R}_{\gamma_{j}}^{d}}
\end{aligned}
$$

The sum of the first and the third term of (6.9) can be treated as in the Dirichlet case and yields the lower order term. The estimate of the other terms are also as similar as the Dirichlet case.

Hence the absolute value of (6.9) is estimated from above by $C_{q, \delta}(1+|\lambda|)^{-\varepsilon}\|f\|_{q}$ $\|u\|_{q^{\prime}}$ for some $\varepsilon>0$.

For the third part, we can treat the Dirichlet and Neumann parts in the same way. We have

$$
\begin{aligned}
& \left(\nabla P\left(\sum_{j=1}^{N} \psi_{j} R_{\gamma_{j}, \lambda} \varphi_{j} f\right)-\nabla\left(\sum_{j=1}^{N} \psi_{j} G_{\gamma_{j}, \lambda} \varphi_{j} f\right), \nabla p_{2}\right)_{\Omega} \\
& =\left(v(\Delta-\nabla \operatorname{div})\left(\sum_{j=1}^{N} \psi_{j} R_{\gamma_{j}, \lambda} \varphi_{j} f\right), \nabla p_{2}\right)_{\Omega}-\left(\nabla\left(\sum_{j=1}^{N} \psi_{j} G_{\gamma_{j}, \lambda} \phi_{j} f\right), \nabla p_{2}\right)_{\Omega}
\end{aligned}
$$

For the estimate of the first term of the right-hand side of (6.10), one uses the following estimate

$$
\begin{aligned}
\left\|(\Delta-\nabla \operatorname{div}) \psi_{j} R_{\gamma_{j}, \lambda} \varphi_{j} f\right\|_{W_{q}^{-\varepsilon}(\Omega)} & \leq C_{q, \delta}\left\|\psi_{j} R_{\gamma_{j}, \lambda} \varphi_{j} f\right\|_{W_{q}^{2-\varepsilon}(\Omega)} \\
& \leq C_{q, \delta}\left\|R_{\gamma_{j}, \lambda} \varphi_{j} f\right\|_{W_{q}^{2-\varepsilon}\left(\mathbb{R}_{\gamma_{j}}^{d}\right)} \\
& \leq C_{q, \delta}\langle\lambda\rangle^{-\frac{\varepsilon}{2}}\left\|\varphi_{j} f\right\|_{L^{q}\left(\mathbb{R}_{\gamma_{j}}^{d}\right)} \\
& \leq C_{q, \delta}\langle\lambda\rangle^{-\frac{\varepsilon}{2}}\|f\|_{L^{q}(\Omega)},
\end{aligned}
$$


where $0<\varepsilon<1$, together with the embedding $W_{q}^{\varepsilon}(\Omega) \hookrightarrow W_{q}^{1}(\Omega)$ for $0<\varepsilon<1$ and the fact that the dual of $W_{q}^{s}(\Omega)$ is $W_{q^{\prime}}^{-s}(\Omega)$ for all $s \in\left(-\frac{1}{q}^{\prime}, \frac{1}{q}\right)$. The second term of the right-hand side of (6.10) can be estimated in the same way as the first term. Thus, combining the previous estimates, we have shown (6.5).

Next let $E: W_{q}^{1-\frac{1}{q}}\left(\Gamma_{2}\right)^{d} \rightarrow W_{q}^{2}(\Omega)^{d}$ be a bounded operator such that $\left.E a\right|_{\Gamma_{1}}=0$ and $\left.T_{1}^{\prime} E a\right|_{\Gamma_{2}}=a$ as well as

$$
\langle\lambda\rangle\|E a\|_{L^{q}(\Omega)}+\left\|\nabla^{2} E a\right\|_{L^{q}(\Omega)} \leq C\left(\|a\|_{W_{q}^{1-\frac{1}{q}}\left(\Gamma_{2}\right)}+\langle\lambda\rangle^{\frac{1}{2}-\frac{1}{2 q}}\|a\|_{L^{q}\left(\Gamma_{2}\right)}\right) .
$$

We note that the existence of such an operator follows from Lemma 7. Hence

$$
\widetilde{R}_{\lambda} f=R_{\lambda} f-E S_{\lambda}^{\prime} f
$$

satisfies

$$
(\lambda-\operatorname{div}(v \nabla \cdot)+\nabla P) \widetilde{R}_{\lambda} f=f+S_{\lambda} f
$$

as well as $\left.\widetilde{R}_{\lambda} f\right|_{\Gamma_{1}}=\left.T_{1}^{\prime} \widetilde{R}_{\lambda} f\right|_{\Gamma_{2}}=0$, where $S_{\lambda}$ satisfies the estimate as in (6.4).

Since $S_{\lambda} \rightarrow 0$ in $\mathcal{L}\left(L^{q}(\Omega)^{d}\right)$ as $|\lambda| \rightarrow \infty, \lambda \in \Sigma_{\delta}$, there is some $R>0$ such that $\left(I+S_{\lambda}\right)^{-1}$ exists for all $\lambda \in \Sigma_{\delta}$ with $|\lambda| \geq R$. Moreover,

$$
\left(I+S_{\lambda}\right)^{-1}=I+S_{\lambda}^{\prime} \text { with }\left\|S_{\lambda}^{\prime}\right\|_{\mathcal{L}\left(L^{q}(\Omega)\right)} \leq C(1+|\lambda|)^{-\varepsilon}
$$

by a standard Neumann series argument. If we substitute $f$ by $\left(I+S_{\lambda}\right)^{-1} f$ in the equation, we have $\left(\lambda+A_{q}\right) \widetilde{R}_{\lambda}\left(I+S_{\lambda}\right)^{-1} f=f$ with $\left.\widetilde{R}_{\lambda}\left(I+S_{\lambda}\right)^{-1} f\right|_{\Gamma_{1}}=0$ and $\left.T_{1}^{\prime} R_{\lambda}\left(I+S_{\lambda}\right)^{-1} f\right|_{\Gamma_{2}}=0$. Hence there exists $R>0$ such that $\left(\lambda+A_{q}\right)$ is surjective for all $\lambda \in \Sigma_{\delta}$ with $|\lambda| \geq R$. Hence, if we show that there exists $R^{\prime}$ such that $\mathcal{N}\left(\lambda+A_{q}\right)=0$ for $\lambda \in \Sigma_{\delta}$ with $|\lambda| \geq R^{\prime}$, we know that $\lambda+A_{q}$ is bijective for $\lambda \in \Sigma_{\delta}$ with $|\lambda| \geq \max \left(R, R^{\prime}\right)$. We need the following lemma.

Lemma 14 Let $\Omega \subseteq \mathbb{R}^{d}, d \geq 2$ and $1<q<\infty$ be as in Assumption 1. If $\lambda+A_{q^{\prime}}$ is surjective for a certain range of $\lambda \in \mathbb{C} \backslash(-\infty, 0]$, then there is no non-trivial solution of (1.5) for the same range of $\lambda$.

Proof Let $f \in L^{q^{\prime}}(\Omega)^{d}$ be arbitrary and let $u \in \mathcal{D}\left(A_{q^{\prime}}\right)$ such that $\left(\lambda+A_{q^{\prime}}\right) u=f$. Then, multiplying $f$ with $\nabla g$, we observe that $\operatorname{div} u \in W_{q^{\prime}}^{1}(\Omega),\left.\operatorname{div} u\right|_{\Gamma_{2}}=0$ solves

$$
-\lambda(\operatorname{div} u, g)-(v \nabla \operatorname{div} u, \nabla g)=(f, \nabla g)
$$

for all $g \in W_{q, \Gamma_{2}}^{1}(\Omega)$. Hence, if $g \in W_{q, \Gamma_{2}}^{1}(\Omega)$ solves (1.5), then $(f, \nabla g)=0$ for all $f \in L^{q^{\prime}}(\Omega)^{d}$ and therefore $\nabla g=0$. Since $\lambda \neq 0$, we get from (1.5) that $g=0$.

Proof of Theorem 1 From the arguments above we know that $\lambda+A_{s}$ for $s=q, q^{\prime}$ is surjective for $|\lambda| \geq R^{\prime}$ with $\lambda \in \Sigma_{\delta}$ for some $R^{\prime}>0$. 
In order to show existence of $\lambda+A_{q}$ for large $\lambda$, it remains to prove $\mathcal{N}\left(\lambda+A_{q}\right)=0$. Using the above lemma, we can conclude that there is no non-trivial solution of (1.5) for the same range of $\lambda$ as before. Now let $u \in \mathcal{N}\left(\lambda+A_{q}\right)$ where $|\lambda| \geq R^{\prime}$ and $\lambda \in \Sigma_{\delta}$. Then we can apply Lemma 4 with $f=g=a=\tilde{p}=0$ to conclude that $u$ solves (1.1)-(1.4) with right-hand side zero. In particular, this implies div $u=0$. In order to show $u=0$, let $f \in L^{q^{\prime}}(\Omega)^{d}$ be arbitrary and let $|\lambda| \geq R^{\prime}$ with $\lambda \in \Sigma_{\delta}$. Let $v \in \mathcal{D}\left(A_{q^{\prime}}\right)$ with $\left(\lambda+A_{q^{\prime}}\right) v=f$. Then

$$
\begin{aligned}
(u, f)_{\Omega} & =\left(u,\left(\lambda+A_{q^{\prime}}\right) v\right)_{\Omega}=\lambda(u, v)_{\Omega}+(2 v D u, D v)_{\Omega} \\
& =\left(\left(\lambda+A_{q}\right) u, v\right)_{\Omega}=0
\end{aligned}
$$

because of (1.10). Since $f \in L^{q^{\prime}}(\Omega)^{d}$ is arbitrary, we get $u=0$. This shows the existence of $\left(\lambda+A_{q}\right)^{-1}$ for $|\lambda| \geq R, \lambda \in \Sigma_{\delta}$. Moreover, because of (6.11),

$$
\left(\lambda+A_{q}\right)^{-1}=R_{\lambda}+S_{\lambda}^{\prime \prime}
$$

where $\left\|S_{\lambda}^{\prime \prime}\right\|_{\mathcal{L}\left(L^{q}(\Omega)\right)} \leq C\langle\lambda\rangle^{-1-\varepsilon}$ for some $\varepsilon>0$. Therefore (1.11) follows from (5.8) and (1.12) follows from (5.10).

Finally, the existence of $h\left(c+A_{q}\right)$ if $c+\Sigma_{\delta^{\prime}} \subset \rho\left(-A_{q}\right)$ and the corresponding estimate (1.14) follows easily form (1.11) and (1.12) using that $\left(\lambda+A_{q}\right)^{-1}$ is uniformly bounded on compact subsets of $\rho\left(-A_{q}\right)$ and a simple shift of the contour. This completes the proof.

\section{Proof of Theorem 2}

Let us assume that $\Omega \subset \mathbb{R}^{d}$ is bounded. Then we know that there exists $\left(\lambda+A_{q}\right)^{-1}$ for any $\lambda \in \Sigma_{\delta}$ such that $|\lambda| \geq R$, where $R$ is a sufficiently large number. Let $\lambda_{0} \in \Sigma_{\delta}$ be such that $\left(\lambda_{0}+A_{q}\right)^{-1}$ exists. Then we have

$$
\begin{aligned}
\left(\lambda+A_{q}\right)\left(\lambda_{0}+A_{q}\right)^{-1} f & =\left\{\left(\lambda-\lambda_{0}\right)+\left(\lambda_{0}+A_{q}\right)\right\}\left(\lambda_{0}+A_{q}\right)^{-1} f \\
& =\left(\lambda-\lambda_{0}\right)\left(\lambda_{0}+A_{q}\right)^{-1} f+f
\end{aligned}
$$

for any $\lambda \in \mathbb{C}$. By Rellich's compactness theorem, we know that the operator $\left(\lambda-\lambda_{0}\right)\left(\lambda_{0}+A_{q}\right)^{-1}$ is compact. Hence we know that $\mathcal{R}\left(\lambda+A_{q}\right)$ has finite codimensions for any $\lambda \in \mathbb{C}$. Thus $\lambda+A_{q}$ is a semi-Fredholm operator for any $\lambda \in \mathbb{C}$. We know also that $\left(\lambda+A_{q}\right)^{-1}$ exists for a certain range of $\lambda \in \mathbb{C}$ as mentioned above. So, using the local invariance of the index of a family of the semi-Fredholm operators, we have ind $\left(\lambda+A_{q}\right)=0$ for any $\lambda \in \mathbb{C}$. To show the existence of the inverse of $\lambda+A_{q}$ for any $\lambda \in \mathbb{C} \backslash(-\infty, 0]$, we only have to show that $\mathcal{N}\left(\lambda+A_{q}\right)=\{0\}$ for the same range of $\lambda$. Moreover, we show that 0 is in the resolvent of $A_{q}$ if $\Gamma_{1} \neq \emptyset$.

First, let $q=2$. Then (A4) is satisfied for any $\lambda \in \mathbb{C} \backslash(-\infty, 0]$. Hence we can apply Lemma 4 with $f=g=a=\tilde{p}=0$ to conclude $\operatorname{div} v=0$ for any $v \in \mathcal{N}\left(\lambda+A_{2}\right)$ and $\lambda \in \mathbb{C} \backslash(-\infty, 0]$. Moreover, if $\lambda=0$, then (1.5) for $g \in W_{2}^{1}(\Omega)$ implies $g \equiv$ const. Therefore $\operatorname{div} v \equiv$ const. for all $v \in \mathcal{N}\left(A_{2}\right)$. Moreover, if $\Gamma_{2} \neq \emptyset$, then $\left.\operatorname{div} v\right|_{\Gamma_{2}}=0$ 
implies $\operatorname{div} v=0$. Finally, if $\Gamma_{2}=\emptyset$, then $\int_{\Omega} \operatorname{div} v d x=\int_{\partial \Omega} v d \sigma=0$, which implies $\operatorname{div} v=0$ again.

Using div $v=0$ for all $v \in \mathcal{N}\left(\lambda+A_{2}\right)$, we conclude further

$$
0=\left(\lambda v+A_{2} v, v\right)_{\Omega}=\lambda(v, v)_{\Omega}+(2 v D v, D v)_{\Omega}
$$

because of (1.10). If $\lambda \in \mathbb{C} \backslash(-\infty, 0]$, then one derives $v=0$ directly. If $\lambda=0$ and $\Gamma_{1} \neq \emptyset$, one also gets $v=0$ by Korn's inequality.

Next we consider the case $q>2$. Since $W_{q}^{2}(\Omega) \hookrightarrow H^{2}(\Omega)$, it follows that $\mathcal{N}\left(\lambda+A_{q}\right) \subseteq \mathcal{N}\left(\lambda+A_{2}\right)=\{0\}$ for all $\lambda \in \mathbb{C} \backslash(-\infty, 0]$ and $\lambda=0$ if $\Gamma_{1} \neq \emptyset$.

Finally, let $1<q<2$ and let $u \in \mathcal{N}\left(\lambda+A_{q}\right)$. Then we have

$$
\begin{aligned}
0= & \left(\lambda v-\operatorname{div}\left(v \nabla v^{T}\right)+\nabla v \cdot \nabla v^{T}+\nabla P v, \nabla g\right) \\
= & -(\lambda \operatorname{div} v, g)-(\nabla v, \nabla v \otimes \nabla g)-(v \Delta v, \nabla g)+(v(\Delta-\nabla \operatorname{div}) v, \nabla g) \\
& +(D v, 2 \nabla v \otimes \nabla g)-\left(\nabla v \cdot \nabla v^{T}, \nabla g\right) \\
= & -(\lambda \operatorname{div} v, g)-(v \nabla \operatorname{div} v, \nabla g)
\end{aligned}
$$

for any $g \in W_{q^{\prime}}^{1}(\Omega),\left.g\right|_{\Gamma_{2}}=0$. Because of $\mathcal{R}\left(\lambda+A_{q^{\prime}}\right)=L^{q^{\prime}}(\Omega)^{d}$ if $\lambda \in \mathbb{C} \backslash(-\infty, 0]$, we can apply Lemma 14 to derive $\operatorname{div} v=0$. If $\lambda=0$ and $\Gamma_{1} \neq \emptyset$, then the arguments in the proof of Lemma 14 show $\nabla \operatorname{div} v=0$. From this one derives $\operatorname{div} v=0$ in the same way as in the case $q=2$. Now let $f \in L^{q^{\prime}}(\Omega)^{d}$ and let $v:=\left(\lambda+A_{q^{\prime}}\right)^{-1} f$, where $\lambda \in \mathbb{C} \backslash(-\infty, 0]$ or $\lambda=0$ if $\Gamma_{1} \neq \emptyset$. - Here we use that the theorem is already proved for the case $q \geq 2$. - Then

$$
\begin{aligned}
(u, f)_{\Omega} & =\left(u,\left(\lambda+A_{q^{\prime}}\right) v\right)_{\Omega}=\lambda(u, v)_{\Omega}+(2 v D u, D v)_{\Omega} \\
& =\left(\left(\lambda+A_{q}\right) u, v\right)_{\Omega}=0
\end{aligned}
$$

due to (1.10). Since $f \in L^{q^{\prime}}(\Omega)^{d}$ is arbitrary, we get $u=0$ if $\lambda \in \mathbb{C} \backslash(-\infty, 0]$ or if $\lambda=0$ and $\Gamma_{1} \neq \emptyset$. This completes the proof.

Acknowledgments The authors are grateful to Gerd Grubb and one anonymous referee for several helpful comments to improve the presentation in this contribution. The second author was supported by a research fellowships of the Japan Society for the Promotion of Science for young scientists.

Open Access This article is distributed under the terms of the Creative Commons Attribution Noncommercial License which permits any noncommercial use, distribution, and reproduction in any medium, provided the original author(s) and source are credited.

\section{References}

1. Abe, T.: On a resolvent estimate of the Stokes equation with Neumann-Dirichlet-type boundary condition on an infinite layer. Math. Methods Appl. Sci. 27(9), 1007-1048 (2004)

2. Abe, T., Shibata, Y.: On a resolvent estimate of the Stokes equation on an infinite layer. J. Math. Soc. Jpn 55(2), 469-497 (2003)

3. Abe, T., Shibata, Y.: On a resolvent estimate of the Stokes equation on an infinite layer, part $2, \lambda=0$ case. J. Math. Fluid Mech. 5, 245-274 (2003) 
4. Abels, H.: On a diffuse interface model for two-phase flows of viscous, incompressible fluids with matched densities. Arch. Ration. Mech. Anal. doi:10.1007/s00205-008-0160-2.

5. Abels, H.: Bounded imaginary powers of the Stokes operator in an infinite layer. J. Evol. Equ. 2, 439457 (2002)

6. Abels, H.: Stokes equations in asymptotically flat domains and the motion of a free surface. PhD thesis, TU Darmstadt, Shaker Verlag, Aachen (2003)

7. Abels, H.: Bounded imaginary powers and $H_{\infty}$-calculus of the Stokes operator in two-dimensional exterior domains. Math. Z. 251(3), 589-605 (2005)

8. Abels, H.: Bounded imaginary powers and $H_{\infty}$-calculus of the Stokes operator in unbounded domains. In: Nonlinear Elliptic and Parabolic Problems. Progr. Nonlinear Differential Equations Appl., vol. 64, pp. 1-15. Birkhäuser, Basel (2005)

9. Abels, H.: Pseudodifferential boundary value problems with non-smooth coefficients. Commun. Partial Differ. Equ. 30, 1463-1503 (2005)

10. Abels, H.: Reduced and generalized Stokes resolvent equations in asymptotically flat layers, part I: unique solvability. J. Math. Fluid. Mech. 7, 201-222 (2005)

11. Abels, H.: Reduced and generalized Stokes resolvent equations in asymptotically flat layers, part II: $H_{\infty}$-calculus. J. Math. Fluid. Mech. 7, 223-260 (2005)

12. Abels, H.: Generalized Stokes resolvent equations in an infinite layer with mixed boundary conditions. Math. Nachr. 279(4), 1-17 (2006)

13. Abels, H.: Diffuse interface models for two-phase flows of viscous incompressible fluids. Lecture Notes, Max Planck Institute for Mathematics in the Sciences, no. 36/2007 (2007)

14. Abels, H., Wiegner, M.: Resolvent estimates for the Stokes operator on an infinite layer. Differ. Integr. Equ. 18(10), 1081-1110 (2005)

15. Adams, R.A.: Sobolev Spaces. Academic Press, New York (1975)

16. Amann, H.: Operator-valued fourier multipliers, vector-valued besov spaces, and applications. Math. Nachr. 186, 5-56 (1997)

17. Bergh, J., Löfström, J.: Interpolation Spaces. Springer, Berlin (1976)

18. Borchers, W., Sohr, H.: On the semigroup of the Stokes operator for exterior domains in $L^{q}$-spaces. Math. Z. 196, 415-425 (1987)

19. Borchers, W., Varnhorn, W.: On the boundedness of the Stokes semigroup in two-dimensional exterior domains. Math. Z. 213, 275-299 (1993)

20. Bothe, D., Prüss, J.: $L_{P}$-theory for a class of non-Newtonian fluids. SIAM J. Math. Anal. 39(2), 379421 (electronic) (2007)

21. Chua, S.K.: Extension theorems on weighted Sobolev space. Indiana Univ. Math. J. 41, 1027-1076 (1992)

22. Danchin, R.: Density-dependent incompressible fluids in bounded domains. J. Math. Fluid Mech. 8(3), 333-381 (2006)

23. Boutet de Monvel, L.: Boundary problems for pseudo-differential operators. Acta Math. 126, 11-51 (1971)

24. Denk, R., Hieber, M., Prüss, J.: $\mathcal{R}$-boundedness, Fourier multipliers and problems of elliptic and parabolic type. Mem. Am. Math. Soc. 166(788), viii+114 (2003)

25. Dore, G., Venni, A.: On the closedness of the sum of two closed operators. Math. Z 196, 189-201 (1987)

26. Farwig, R.: Weighted $L^{q}$-Helmholtz decompositions in infinite cylinders and in infinite layers. Adv. Differ. Equ. 8, 357-384 (2003)

27. Farwig, R., Kozono, H., Sohr, H.: An $L^{q}$-approach to Stokes and Navier-Stokes equations in general domains. Acta Math. 195, 21-53 (2005)

28. Farwig, R., Ri, M.-H.: The resolvent problem and $H^{\infty}$-calculus of the Stokes operator in unbounded cylinders with several exits to infinity. J. Evol. Equ. 7(3), 497-528 (2007)

29. Farwig, R., Ri, M.-H.: Stokes resolvent systems in an infinite cylinder. Math. Nachr. 280(9-10), 10611082 (2007)

30. Farwig, R., Sohr, H.: Generalized resolvent estimates for the Stokes system in bounded and unbounded domains. J. Math. Soc. Jpn 46(4), 607-643 (1994)

31. Farwig, R., Sohr, H.: Helmholtz decomposition and stokes resolvent system for aperture Domains in $L^{q}$-spaces. Analysis 16, 1-26 (1996)

32. Giga, Y.: Analyticity of the semigroup generated by the Stokes operator in $L_{r}$ spaces. Math. Z. 178, 297-329 (1981) 
33. Giga, Y.: Domains of fractional powers of the Stokes operator in $L_{r}$ Spaces. Arch. Ration. Mech. Anal. 89, 251-265 (1985)

34. Giga, Y., Sohr, H.: On the Stokes operator in exterior domains. J. Fac. Sci. Univ. Tokyo Sect. IA Math. 36(1), 103-130 (1989)

35. Giga, Y., Sohr, H.: Abstract $L^{p}$ estimates for the Cauchy problem with applications to the NavierStokes equations in exterior domains. J. Funct. Anal. 102, 72-94 (1991)

36. Grubb, G.: Pseudo-differential boundary problems in $L_{p}$ spaces. Commun. Partial Differ. Equ. 15, 289-340 (1990)

37. Grubb, G.: Functional Calculus of Pseudodifferential Boundary Problems, 2nd edn. Birkhäuser, Basel (1996)

38. Grubb, G., Kokholm, N.J.: A global calculus of parameter-dependent pseudodifferential boundary problems in $L_{p}$ Sobolev spaces. Acta Math. 171(2), 165-229 (1993)

39. Grubb, G., Solonnikov, V.A.: Boundary value problems for the nonstationary Navier-Stokes equations treated by pseudo-differential methods. Math. Scand. 69, 217-290 (1991)

40. Málek, J., Nečas, J., Rokyta, M., Ružička, M.: Weak and measure-valued solutions to evolutionary PDEs. In: Applied Mathematics and Mathematical Computation, vol. 13, vii, 317 p. Chapman \& Hall, London (1996)

41. Kumano-Go, H., Nagase, M.: Pseudo-differential operators with non-regular symbols and applications. Funkcial Ekvac. 21, 151-192 (1978)

42. Ladyženskaja O.A., Solonnikov, V.A.: The unique solvability of an initial-boundary value problem for viscous incompressible inhomogeneous fluids. Zap. Naučn. Sem. Leningrad. Otdel. Mat. Inst. Steklov. (LOMI) 52,52-109, 218-219 (1975). Boundary value problems of mathematical physics, and related questions of the theory of functions, 8

43. Marschall, J.: Pseudodifferential operators with coefficients in Sobolev spaces. Trans. Am. Math. Soc. 307(1), 335-361 (1988)

44. McIntosh, A.: Operators which have an $H_{\infty}$-calculus. In: Miniconference on Operator Theory and Partial Differential Equations. In: Jefferies, B., McIntosh, A., Ricker, W. (eds.) Proceedings of Center Math. Anal. A.N.U., vol. 14, pp. 210-231 (1986)

45. Miyakawa, T.: The Helmholtz decomposition of vector fields in some unbounded domains. Math. J. Toyama Univ. 17, 115-149 (1994)

46. Noll, A., Saal, J.: $H^{\infty}$-calculus for the Stokes operator on $L_{q}$-spaces. Math. Z. 244, 651-688 (2003)

47. Schumacher, K.: A chart preserving the normal vector and extensions of normal derivatives in weighted function. Preprint, TU Darmstadt, No. 2510 (2007)

48. Simader, C.G., Sohr, H.: A new approach to the Helmholtz decomposition and the Neumann problem in $L^{q}$-spaces for bounded and exterior domains. In: Mathematical Problems Relating to the NavierStokes Equation. Ser. Adv. Math. Appl. Sci., vol. 11 , pp. 1-35. World Scientific, River Edge (1992)

49. Solonnikov, V.A.: $L_{p}$-estimates for solutions to the initial boundary-value problem for the generalized Stokes system in a bounded domain. J. Math. Sci. (New York) 105(5), 2448-2484 Function theory and partial differential equations (2001)

50. Solonnikov, V.A.: Estimates of the solution of model evolution generalized Stokes problem in weighted Hölder spaces. Zap. Nauchn. Sem. S.-Peterburg. Otdel. Mat. Inst. Steklov. (POMI) bf 336 (Kraev. Zadachi Mat. Fiz. i Smezh. Vopr. Teor. Funkts. 37),211-238, 277 (2006)

51. Triebel, H.: Interpolation Theory, Function Spaces, Differential Operators. North-Holland, Amsterdam (1978) 